
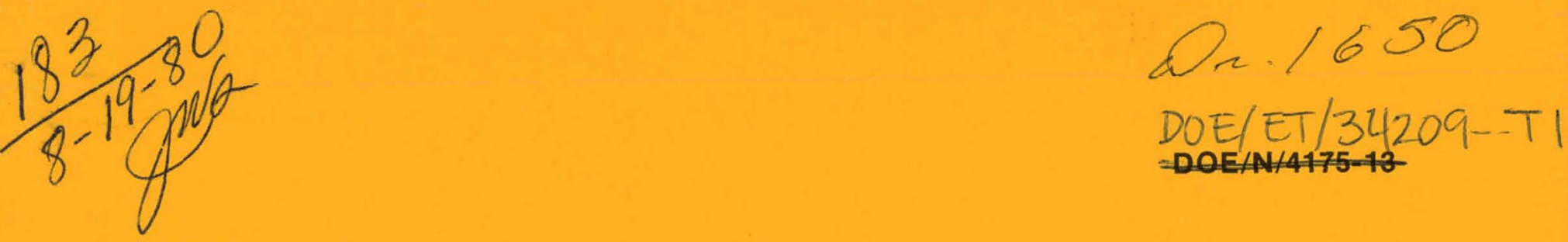

CORRELATION AND SPECTRAL MEASUREMENTS OF FLUCTUATING PRESSURES AND VELOCITIES IN ANNULAR TURBULENT FLOW

FEBRUARY 1980

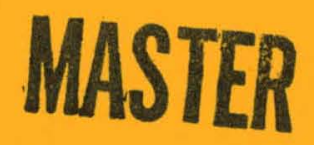

\author{
RANDALL J. WILSON \\ ARGONNE NATIONAL LABORATORY \\ BARCLAY G. JONES \\ RAMENDRA P. ROY \\ UNIVERSITY OF ILLINOIS
}

\begin{abstract}
GENERAL ELECTRIC COMPANY NUCLEAR ENGINEERING DIVISION

175 CURTNER AVENUE

SAN JOSE, CALIFORNIA 95125
\end{abstract}

\author{
PREPARED FOR THE \\ U.S. DEPARTMENT OF ENERGY \\ ASSISTANT SECRETARY FOR NUCLEAR \\ OFFICE OF ENERGY RESEARCH \\ UNDER CONTRACT EN-77-C-02-4175
}




\section{DISCLAIMER}

This report was prepared as an account of work sponsored by an agency of the United States Government. Neither the United States Government nor any agency Thereof, nor any of their employees, makes any warranty, express or implied, or assumes any legal liability or responsibility for the accuracy, completeness, or usefulness of any information, apparatus, product, or process disclosed, or represents that its use would not infringe privately owned rights. Reference herein to any specific commercial product, process, or service by trade name, trademark, manufacturer, or otherwise does not necessarily constitute or imply its endorsement, recommendation, or favoring by the United States Government or any agency thereof. The views and opinions of authors expressed herein do not necessarily state or reflect those of the United States Government or any agency thereof. 


\section{DISCLAIMER}

Portions of this document may be illegible in electronic image products. Images are produced from the best available original document. 


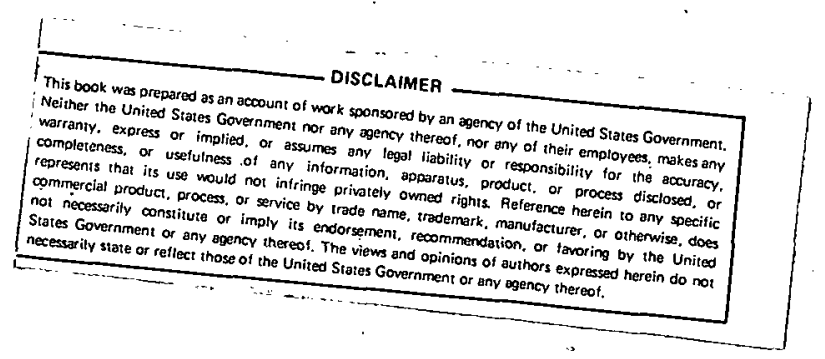

$$
\begin{aligned}
& \text { DOE/ET/342O9--T/ } \\
& \text { DOE/N/4175-13 }
\end{aligned}
$$

Distribution Category UC-78

\title{
CORRELATION AND SPECTRAL MEASUREMENTS OF FLUCTUATING PRESSURES AND VELOCITIES IN ANNULAR TURBULENT FLOW
}

\author{
MANUSCRIPT COMPLETED \\ FEBRUARY 1980
}

RANDALL J. WILSON

ARGONNE NATIONAL LABORATORY

BARCLAY G. JONES

RAMENDRA P. ROY

UNIVERSITY OF ILLINOIS

\author{
GENERAL ELECTRIC COMPANY \\ NUCLEAR ENGINEERING DIVISION \\ 175 CURTNER AVENUE \\ SAN JOSE, CALIFORNIA 95125
}

\author{
PREPARED FOR THE \\ U.S. DEPARTMENT OF ENERGY \\ ASSISTANT SECRETARY FOR NUCLEAR \\ OFFICE OF ENERGY RESEARCH \\ UNDER CONTRACT EN-77-C=02-4175
}




\section{DISCLAIMER}

This book was prepared as an account of work sponsored by an agency of the United States Government. Neither the United States Government nor any agency thereof, nor any of their employees, makes any warranty, express or implied, or assumes any legal liability or responsibility for the accuracy, completeness, or usefulness of any information, apparatus, product, or process disclosed, or represents that its use would not infringe privately owned rights. Reference herein to any specific commercial product, process, or service by trade name, trademark, manufacturer, or otherwise, does not necessarily constitute or imply its endorsement, recommendation, or favoring by. the United States Government or any agency thereof. The views and opinions of authors expressed herein do not necessarily state or reflect those of the United States Government or any agency thereot.

Printed in the United States of Alritilcid Available from National Technical Information Service U.E. Dopartment of remmerre 5265 Port Royal Road Springficld, $\vee \wedge 22161$

Price: Code A07

Microfiche $\$ 3.50$ 
2. EXPERIMENTAL EQUIPMENT AND CALIBRATION

2.5 Instrumentation

3. DATA ANALYSIS TECHNIQUES

3.1 Inclined Hot-Wire Response for Velocity Measurements

3.2 Techniques for Pressure Measurements and Pressure-Pressure Correlation Measurements

3.3 Techniques for Pressure-Velocity Correlation Measurements

4. SINGLE POINT MEASUREMENTS

4.1 Single Point Measurements

4.2 Single Point Turbulent Velocity Measurements

4-4

4.3 Single Point Fluctuating Static Pressure Measurements

5. TWO POINT MEASUREMENTS

5.1 Pressure-Pressure Correlations

5.2 Pressure-Velocity Correlations

6. SUMMARY, CONCLUSIONS AND RECOMMENDATIONS

FOR FUTURE RESEARCH

7. REFERENCES 


\section{LIST OF ILLUSTRATIONS}

Figure

$\underline{\text { Title }}$

Page

2-1 Schematic Diagram of the Test Flow Facility Used in This Study

$2-3$

2-2 A Schematic of the Test Section Showing the Axial Measurement Ports, the Rotating Section and One of the Three Rods Used

to Hold the Test Section in Place

$2-4$

2-3 End View and Side View of the Tongue and Groove Arrangement Used to Join the Test Section While Allowing Angular Rotation

2-4 Spider Arrangement Used to Align and Support the 1/2-in. Diameter Center Rod at the Flanges

2-5 Geometry Orientation in the Test Section as it was Defined for This Study, Including the Three Orthogonal Separations of the Transducers

2-6 Detailed Drawing of the Wall Pressure Transducer as Given by the Manufacturer, Celesco Industries, Inc.

$2-10$

2-7 End View of Test Section Showing the Individual Wall

Pressure Transducers as Mounted in the Test Section and the Designated Electronics Associated With Each

2-8 Detailed Diagram of the Mounting of the Wall Pressure Transducer in the Center Rod. The Cavity was Filled With Porous Foam

2-9 Block Diagram of the Electronics and Instrumentation Used in the Wall Pressure Transducer Frequency Response Calibration 2-14

2-10 Frequence Response of the Wall Pressure Transducers ' 2-15

2-11 Wall Pressure Transducer Output as a Function of RMS Pressure Level for Sensor No.733. The Slope is 0.384 Volts/in. $\mathrm{H}_{2} \mathrm{O}$

2-12 Frequency Response of the Inner Wall Pressure Transducer Mounted in the Center Rod With and Without the Notch Filter

2-13 Design of the Notch Filter Used to Flatten the Frequency hesponse of the Inner Wall Pressure Transducer

2-14 Schemat1c Drawing of the Two Bleed Pressure Transducers With the Essential External Dimensions

2-15 Bleed Pressure Transducer Static Calibration Data Yielding the Sensitivitiy of the Sensors at Low Frequency $\left(25.1 \mathrm{mV} / \mathrm{in}\right.$. $\mathrm{H}_{2} \mathrm{O}$ for $1412 \mathrm{G}$ and $21.4 \mathrm{mV} / \mathrm{in}$. $\mathrm{H}_{2} \mathrm{O}$ for $\left.1412 \mathrm{~J}\right)$ 


\section{LIST OF ILLUSTRATIONS (Continued)}

Figure

Title

Page

2-16 Block Diagram of the Electronics and Instrumentation for the Frequency Response Calibration of the Bleed Pressure Transducers

2-17 Frequency Response of the Bleed Pressure Transducers With and Without the RC Correction Network

2-18 RC Correction Network for Flattening the Frequency

Response of the Bleed Pressure Transducers

2-19 Instrument Holder Shown Mounted on the Test Section

With a Bleed Pressure Transducer in Place

2-20 Linearized Calibration Curve of X-Probe 1249G-T1.5

2-21 Yaw Response of X-Probe 1249G-T1.5 With $\left(a_{1}\right)_{0}=38^{\circ}$ and $\left(a_{2}\right)_{0}=42^{\circ}$ for a Relative Angle of $40^{\circ}$ Between the Two Wires and the Mean Velocity o

3-1 Vector Representation of Hot-Wire and Instantaneous

Velocity Vectors

4-1 Average Velocity. Profile at a Mean Velocity of $200 \mathrm{ft} / \mathrm{sec}$

$4-2$

1-2 Law of the Wall Mean Velocity Profile

$4-3$

4-3 Block Diagram of the Velocity Data Acquisition System

$4-5$

4-4 Reynolds Stress Distribution 4-6

4-5 Radial Distribution of the Turbulent Axial Velocity Component 4-9

4-6 Radial Distribution of the Turbulent Radial Velocity Component 4-10

4-7 Power Spectral Density of the Axial Component of the Turbulent Velocity Field at $\bar{U}=200 \mathrm{ft} / \mathrm{sec}$

4-8 Power Spectral Density of the Radial Component of the Turbulent Velocity Field at $\vec{U}=200 \mathrm{ft} / \mathrm{ser}$.

$4-12$

4-9 Block Diagram of the Wall Pressure Data Acquisition System

$4-14$

4-10. Block Diagram of the In-Stream Pressure Data Acquisition System 4-15

4-11 Whessure Intensity Level as a Function of

Mean Flow Velocity

4-12 Power Spectral Density of a Single Wall Pressure Transducer and That of the Acoustic Pressure Field 


\section{LIST OF ILLUSTRATIONS (Continued)}

Figure

Title

$\underline{\text { Page }}$

4-13 Power Spectral Density of the Hydrostatic Wall Pressure and the In-Stream Pressure Showing the $3.4 \mathrm{KHz}$ Acoustic Peak Which Could Not Be Electronically Removed

4-14 Power Spectral Density of the Hydrostatic Pressure Fluctuations at the Inner Wall for Several Different Mean Flow Velocities

4-15 Power Spectral Density of the Hydrostatic Pressure Fluctuations at the Outer Wall for Several Different Mean Flow Velocities

4-16 Profile of the In-Stream Hydrostatic Pressure Fluctuation Intensity Level

5-1 Correlation between the Inner Wall Pressure Fluctuations and the In-Stream Pressure Fluctuations as a Function of Radial Separation

5-2 Correlation between $t_{n}$ Outer Wall Pressure Fluctuations and the In-Stream Pressure Fluctuations as a Function of Radial Separation

5-3 Azimuthal Pressure-Pressure Correlation between In-Stream Pressure Transducers at $R^{*}=0.11$

5-4 Azimuthal Pressure-Pressure Correlation between In-Stream Pressure Transducers at $R^{*}=0.25$

5-5 Azimuthal Pressure-Pressure Correlation between In-Stream Pressure Transducers at $R^{*}=0.66$

5-6 Azimuthal Pressure-Pressure Correlation between In-Stream Pressure Transducers at $R^{*}=0.89$

5-7 Azimuthal Pressure-Pressure Correlation between the In-Stream Bleed Pressure Transducer at $\mathrm{R}^{*}=0.11$ and the Inner Wall Pressure Transducer

5-8 Azimuthal Pressure-Pressure Correlation between the In-Stream Bleed Pressure Transducer at $R^{*}=0.89$ and the Outer Wall Pressure Transducer

5-9 Axial Pressure-Pressure Correlation between the Wall Pressure Transducers and the Bleed Pressure Transducer at $R^{*}=0.11$ for the Inner Wall and $R^{*}=0.89$ for the Outer Wall 


\section{LIST OF ILLUSTRATIONS (Continued)}

Figure

Title

$\underline{\text { Page }}$

5-10 Delay Time to Maximum Pressure-Pressure Correlation

for Axial Separation at Both Walls of the Flow Channel, which Results in a Convection Velocity, $U_{C}$, of $204+1-$ $5 \mathrm{ft} / \mathrm{sec}$

5-11 Cross-Correlations between the Axial Velocity Component and the Inner Wall Pressure as a Function of Delay

Time for Different Axial Separations

5-12 Cross-Correlations between the Axial Velocity Component and the Uuter Wall Pressure as a Function of Delay Time for Different Axial Separaliuns

5-13 Cross-Correlations between the Radial Velocity Component and the Inner Wall Pressure as a Funclion of Delay Time for Different Axial Separations

5-14 Cross-Correlations between the Radial Velocity Component and the Outer Wall Pressure as a Function of Delay Time for Different Axial Separations

5-15 The Magnitude of the Maximum Correlation between the Axial Velocity Component and Wall Pressure for Increasing Axial Separation Distances

5-16 The Magn1tude of the Maximum Correlation between the Radial Velocity Component and Wall Pressure for Increasing Axial Separation Distances

5-17 Axial Separation Distance Versus Delay Time to Maximum Pressure-Velocity Correlation at the Inner Wall

5-18 Axial Separation Distance Versus Delay Time to Maximum Pressure-Veloc1ty Correlation at the Outer Wall

5-19 Maximum Correlation between the Inner Wall Pressure and the Axial Velooity Componcnt ao a Function of Radial Separation Distance

5-20 Maximum Correlation between the Outer Wall Pressure and the Axial Velocity Component as a Function of Radial Separation Distance

5-21 Maximum Correlation between the Inner Wall Pressure and the Radial Velocity Component as a Function of Radial Separation Distance 
5-22 Maximum Correlation between the Outer Wall Pressure and the Radial Velocity Component as a Function of Radial Separation Distance

5-23 Maximum Correlation between the Inner Wall Pressure and the Axial Velocity Component as a Function of Azimuthal Separation Angle

5-24 Maximum Correlation between the Outer Wall Pressure and the Axial Velocity Component as a Function of Azimuthal Separation Angle

5-25 Maximum Correlation between the Inner Wall Pressure and the Radial Velocity Component as a Function of Azimuthal Separation Angle

5-26 Maximum Correlation between the Outer Wall Pressure and the Radial Velocity Component as a Function of Azimuthal Separation Angle

5-27 Delay Time to Maximum Pressure-Velocity Correlation as a Function of Radial Position

5-28 Veloc1ty Field as Estimated from the PressureVelocity Correlations Using Linear Estimation Theory 
An experimental study of the fluctuating velocity field, the fluctuating static wall pressure and the in-stream fluctuating static pressure in an annular turbulent air flow system with a radius ratio of 4.314 has been conducted. The study included direct measurements of the mean velocity profile, turbulent velocity field; fluctuating static wall pressure and in-stream fluctuating static pressure from which the statistical values of the turbulent intensity levels, power spectral densities of the turbulent quantities, the cross-correlation between the fluctuating static wall pressure and the fluctuating statice pressure in the core region of the flow and the crosscorrelation between the fluctuating static wall pressure and the fluctuating velocity field in the core region of the flow were obtained.

The velocity measurements were made with $X$-probe hot-wire sensors utilizing standard constant temperature hot-wire anemometry techniques. Both mean flow and turbulent velocity measurements were made to ensure that the flow facility gave a fully developed turbulent flow field which was consistent with information in the literature.

The wall pressure measurements were made with small (0.034-square in.) piezoelectric transducers. A three-sensor, signal subtraction, data analysis method using coherence techniques was developed to separate the superimposed local pressure fluctuations and acoustically transmitted noise. This analysis method was shown to adequately isolate the local pressure fluctuation informaliurl al eavh wall of the flow channel.

The in-stream fluctuating static pressure measurements were made with a miniature bleed-type pressure transducer. The pressure transducer was shown to be smalzer than the turbulent velocity or pressure scales, to respond linearly to pressure, have a flat frequency response to $10 \mathrm{KHz}$ and have a velocity induced error of less than 5\%. The signal analysis method used for the wall pressure measurements was extended to include the measurement of the in-stream fluctuating. static pressure. 
The effect of the turbulent core region of the flow field on the wall pressure fluctuations was studied by cross-correlating the in-stream pressure fluctuations with the wall pressure fluctuations. The data analysis technique used for single point measurements was extended to these two point measurements to isolate the correlation between the local fluid pressure fluctuations due to the fluid turbulence. A similar approach was used for the cross-correlation of the fluctuating static wall pressure and the fluctuating velocity field.

The results of the experimental measurements are compared with existing experimental and numerical information on turbulent annular flow fields and wall pressure statistics. The wall pressure statistices have been shown to bo closely related to the flow field statistics. The strongest relationship was shom to orour between the fluid shoar otress and the wat. pressure. The experimentally measured pressure statistics were also. shown to be in good agreement with numerical work being dore in Karlsmihe, West Germany. The resulting integral length scales were shown to be significantly larger than the boundary layer thickness which indicates the necessity of relating wall pressure statistics to specific flow fields turbulence rather than utilizing information from the boundary layer region orly.

\section{ACKNOWLEDGEMENTS}

One of the authors (Randali J. Wilson) is sincerely grateful to his advisor, Professor B. G. Jones, for his guidance throughout this investigation. Hias encouragement and assistance is deeply appreciated.

This work was performed at the University of Illinois and was funded by the Reserres Board of the University of Illinois (Grants 1-40-26-09-301 and 1-40-23-33-34\%), by Argonne National Laboratory (Grant ANL-SBC-31-109-38-3987) and by the Nuclear Engineering Program, for which the authors are most appreciative. 


\section{INTRODUCTION}

The problem of flow-induced vibrations is an area which has become quite important to the nuclear industry in recent years. Nuclear reactor systems include many types of flexible component structures which are subject to turbulent fluid flow. These stochastic velocity and pressure fluctuations can act as excitation mechanisms for. flow-induced vibrations. Fluid excitation forces include those due to: wake flows, buffeting, boundary layers, acoustic noise, and fluid-structure coupling. Flow-induced vibrations can cause nuclear reactor component failures ${ }^{1}$ which can result in large financial losses for power companies. It has been estimated 2,3 that the second greatest cause of nuclear plant outage is the repair of components dainaged by excessive vibration.

It would be very beneficial to be able to design reactor components and flow systems such that flow-induced vibrations are eliminated, or at least minimized. The necessary design criteria can only be obtained with an adequate knowledge and understanding of the flow field turbulence which generates the excitation forces. This information can only be obtained thruugh fundamental studies designed to give a better understanding of the interaction of the flow field turbulence and the flow field containment.

The two primary flow-induced vibrations, found in nuclear reactors are paralleiflow-induced vibrations and cross-flow-induced vibrations. Only the first of these, parallel-flow-induced vibrations, will be dealt with in this report as they are the most common and least understood vibrations occurring in nuclear reactor systems. There is, however, a great amount of work being done on crossflow-induced vibrations and their mechanisms are not fully understood.

Previous and current research into parallel-flow-induced vibrations has taken many approaches. Much of this work has been reviewed and summarized in the 1iterature. 4-10 The experimental approaches range. from complete scale models of reactor internals, to a simply supported single pin in a pipe. Many of the experiments in more complex geometries ${ }^{11-25}$ try to monitor the 
structural vibrations and correlate the displacement to the fluid properties, flow velocity and channel dimensions. This engineering approach has been useful in specific flow geometries and conditions, but does not lend itself either to fundamental understanding of the mechanism or a general design solution. The single pin, or annular flow geometry, is a simplified flow channel model which allows better control of the flow conditions. Several parallel-flow-induced vibration experiments ${ }^{26-40}$ have been run with this geometry. The most useful information in terms of design work is probably that of Reavis. ${ }^{39}$ His semi-empirical equation for fuel rod displacement contains several parameters which allow it to be used in many design situations as long as a siguiff,cant amount of conservatism is empluyed.

Dlrect englneering approaches allow one to make a qualitative estimate of the vibration problems associated with a specific design. They do not, however, make a significant contribution to the understanding of the flow mechanisms which result in flow-induced vibrations. A trial and error approach with this method can result in some general design criteria which can reduce the chances of flow-induced-vibrations occurring.

Un the more fundamental side of flow-induced-vibrations much work has been done in boundary layer flows. ${ }^{41-88}$ These include pipe flow, channcl flow, and large boundary layers in wind tunnels, Much of this work consists of fluctuating static pressure measurements on the wall on the flow channel. 42,43,46-51,54,55, 59-61,66,68,73-76,78, 80-82,84 This work includes the measurement of the rootmean-square (RMS) level of the fluctuating static pressure, the power epootra1 density (PSD) of the fluctuating static pressure and mapping of the pressurepressure correlation on the surfare of the flow channel. Exleusive work has also been done in measuring the velocity profiles, RMS levels and PSD's in the boundary layer. $41,44,45,52,53,36-58,62-65,67,69,70,79,83,85-87,88$ There are also two studies on the correlation between the velocity components in the boundary layer and'the fluctuating static wall pressure beneath the boundary layer. $^{72,77}$ 
A11 of these boundary layer investigations give some insight into the processes of turbulence near the wall region and its relationship to the wall pressure. They do not, however, investigate the contribution to the wall pressure fluctuations from the turbulent flow field beyond the boundary layer, as they have been limited to investigating only that contribution to the wall pressure fluctuations from within the turbulent boundary layer. Since pressure is an integral quantity, it would appear that there is a need to look at the contribution to the wall pressure fluctuations from the flow region outside the boundary layer. The volume of contribution to the wall pressure fluctuations should be as large as the extent of a significant pressure-pressure correlation. Therefore, a correlation of the wall pressure with flow field pressure from outside the boundary layer would indicate a contribution to the wall pressure from the turbulent core in an internal flow field.

Some recent direct numerical simulations of turbulent channel flow by Grotzbach and Schumann $58,59,71$ have included the total flow field in the contribution of the fluctuating static wall pressure. Their resultant wall pressure statistics agree quite well with the experimental measurements noted above. Much information can be learned from these computer models, but they must be checked, whenever possible, against experimental results to ensure that the information they generate accurately models reality.

This investigation was planned and designed with the idea of cross-checking the above numerically simulated turbulent flow statistics and correlations, while simultaneously yielding new insight and information on the contribution of the turbulent. core flow on the ultimate wall pressure fluctuations which drive the structural vibrations. This includes the measurement of both the fluctuating velocity components and the fluctuating static pressure in the flow field and the correlation of each with the wall pressure fluctuations. 


\section{EXPERIMENTAL EQUIPMENT AND CALIBRATION}

\subsection{FLOW FACILITY}

The flow system selected for this experiment was an open cycle air flow loop. Air was selected over water as the fluid because it was available in large flow quantities, and measurements of fluctuating velocities and static pressures with available hot-wire anemometry sensors could be performed more easily and without sensor development. The air supply was provided by a Gardner-Denver rotary compressor. It provided up to 600 standard cubic feet per minute of clean, dry air at $90 \mathrm{psig}$.

The flow system was designed to perform under a wide range of flow velocities while maintaining flow parameters as close as possible to those used by Schumann ${ }^{71}$ under our operating budget constraints. Schumann's simulated flow system utilized an incompressible fluid flowing in an annular channel with an outer-to-inner radius ratio of 5.0 and a Reynolds number based on the hydraulic diameter of 300,000 .

Other basic system requirements were that the flow be fully developed, the channel sizes and materials allow probes to be inserted into the flow and to be traversed across the flow channel, and that the system be designed to minimize acoustic noise production. The system was also to be designed such that no vibrations were present. This allowed for the investigation of the fluid mechanics which generates the wall pressure without the interference of the coupled wall-fluid motion.

Acoustic noise generation is a difficult and complex problem. Acoustic noise can be generated by turbulent flow-through restrictions, expansions, valves and elbows. Pressure transducers which are used to measure the local fluid pressure fluctuations are equally sensitive to an acoustic pressure fluctuation. In ordcr to ensure that the analyzed pressure signal is truly that of the hydrostatic pressure fluctuations it is necessary to either eliminate all of the acoustic pressure fluctuations or develop a method of data processing which separates the two sources. In the last case it is desirable to reduce the acoustic pressure level to the fullest extent possible. 
The acoustic noise problem is one which was first noted by Willmarth and Wooldridge ${ }^{76}$ and is noted by most investigators of wall pressure measurements. There has been a considerable amount of work done on controlling acoustic noise generation in pipe systems. ${ }^{89-119}$ A comprehensive review by Reethof ${ }^{119}$ is a useful summary of acoustic noise control methods.

The supply air delivered by the Gardner-Denver compressor is regulated by a Fisher 667-ED control valve using a remote panel loader: Porous plates having about $18 \%$ open area are located directly downstream of both the regulating valve and the manifold. Thess are intended to reduce the propagation of the acoustic noise generated by the upstreall valves and filow restrictions. The air then enters a 230-gallon receiving tank, passing by an acoustically lined baffle plate and through a filter. From the receiving tank the air passes through a flexible 3-Inch hose to the entrance of the flow facility. The flexible hose connects to a 4-in. PVC line (Figure 2-1) where the flow passes through an orifice plate which is used to meter the flow rate. The top section is made of 4-in. diameter PVC and four, 45-degree elbows which are tubed with $1 / 2-i n$. tygon tubing to control the flow through them. On the downcomer slde is a Burgess-Manning BMSS-4 acoustic silencer which is uged to reduce the acoustic noise level in the flow channel. At the exit of the muffler is a section of stainless steel (SS) 304 which reduces the 4-in. diametcr silencer exit tu a nominal 2-in. diameter. The 1/2-in. stainless steel rod which forms the completed annular flow channel also begins at this point. This flow development section is $10 \mathrm{ft}$ long which is approximately 72 hydraulic diameters and contains screening at the entrance to help develop the flow field. It has been indicated ${ }^{120}$ that fully developed turbulent flow in an annular flow clannel can be developed in as little as 34.5 diameters. This development section connects to the $4-f f$ long toot ocetion whilu in curn connects to the $3-\mathrm{ft}-10 \mathrm{ng}$ exit section where the air is dumped into the local environment.

The test section physicai dimensions are shown in Figurc 2-2. "It was machined from a 4-ft long, 3-in. diameter cylinder of transparent lucite. "The inner diameter was bored to 2.157 in. to match the 2-in. diameter schedule 10 piping used in the development section. The test section was machined in three 


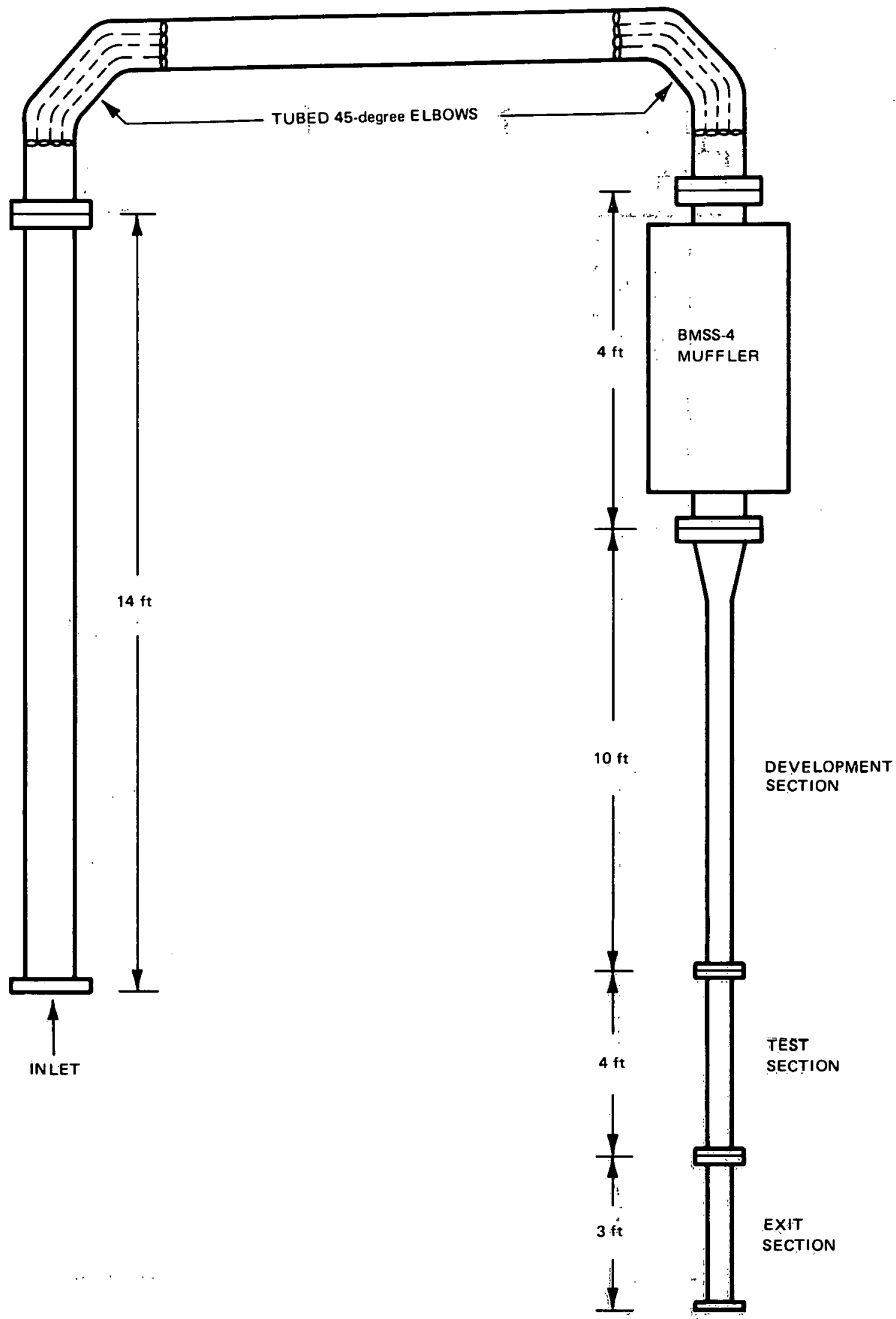

Figure 2-1. Schematic Diagram of the Test Flow Facility Used in this Study. 


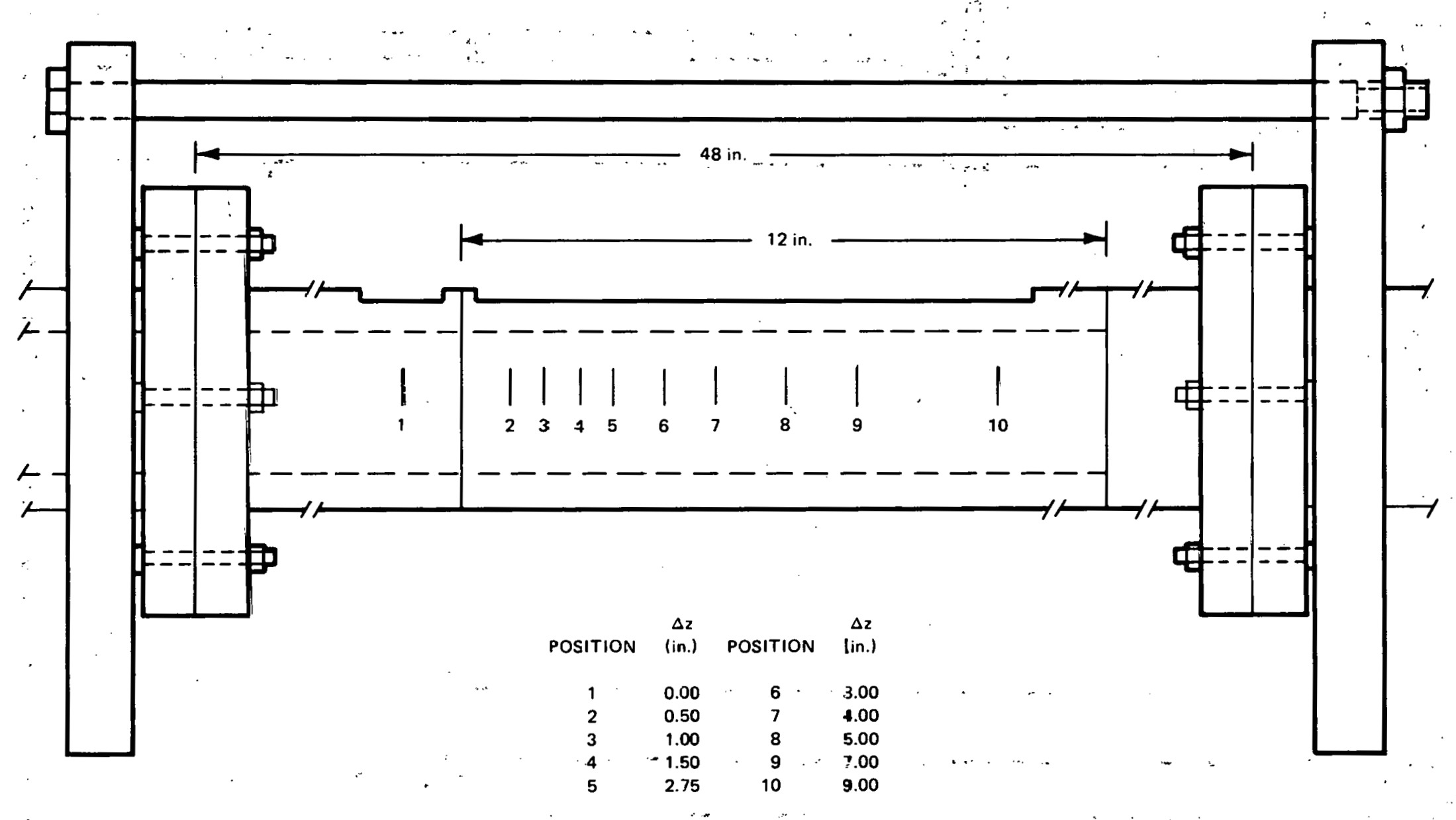

Figure 2-2. A Scheratic of the Test Section Showing the Axial Measurement Ports, the Rotating section and one of the Three Rods Used to Hold the Test Section in Place 
separate sections and was fitted together with a tongue and groove arrangement and sealed with an 0-ring, Figure 2-3. When mounted in the loop it was held in place with two large flanges and pulled together with three threaded rods as shown in Figure 2-2. This configuration allows the center section to be freely rotated, making it possible to azimuthally separate probes at any desired separation angle. Probe mounting ports were machined at 10 separate locations as noted in Figure 2-2 and two wall pressure transducer mounts were machined at axial position 1 . When not in use, mounting ports were plugged with a piece of lucite machined to seal the port and mount flush with the inner wall.

The center rod runs the full extent of the 2-in. diameter downcomer side of the flow loop. It is a 0.50-in. o.d. 304 sS tube with a 0.035-in. wall thickness. This wall thickness was sufficient to prevent measurable vibrations from occurring. The rod is supported and aligned at each flange with a three prong spider arrangement shown in Figure 2-4. The three support rods were aerodynamically shaped to minimize flow distrubances.

The resultant flow system gives a well controlled, fully developed turbulent air flow facility. Excessive elbows, valves and restrictions have been eliminated to reduce the acoustic noise production. The radius ratio of the flow channel is 4.314 with a hydraulic diameter of $1.657 \mathrm{in}$. The system flow velocity range is 0 to $425 \mathrm{ft} / \mathrm{sec}$ with a maximum Reynolds number of 300,000 . The radius ratio is slightly less than Schumann's and the Reynolds number range barely includes that used by Schumann. Due to the above mentioned acoustic noise problems the mean flow velocity used for most all of the measurements was $200 \mathrm{fL} / \mathrm{sec}$ with a Reynolds number of 160,000 . The test section geometry is defined in Figure 2-5.

The mean flow velocity was measured with a sharp edged orifice plate with an orifice to pipe diameter ratio of 0.62 . Pressure taps were located 1 diameter upstream and $1 / 2$ diameter downstream of the orifice plate and were 0.25 in. in diameter. A U-tube waler manometer was used to monitor the differential pressure. The orifice plate was calibrated with a pitot tube which was located in the test section during the calibration procedure. Mean velocity versus pressure drop across the orifice plate was least squares fitted with a 

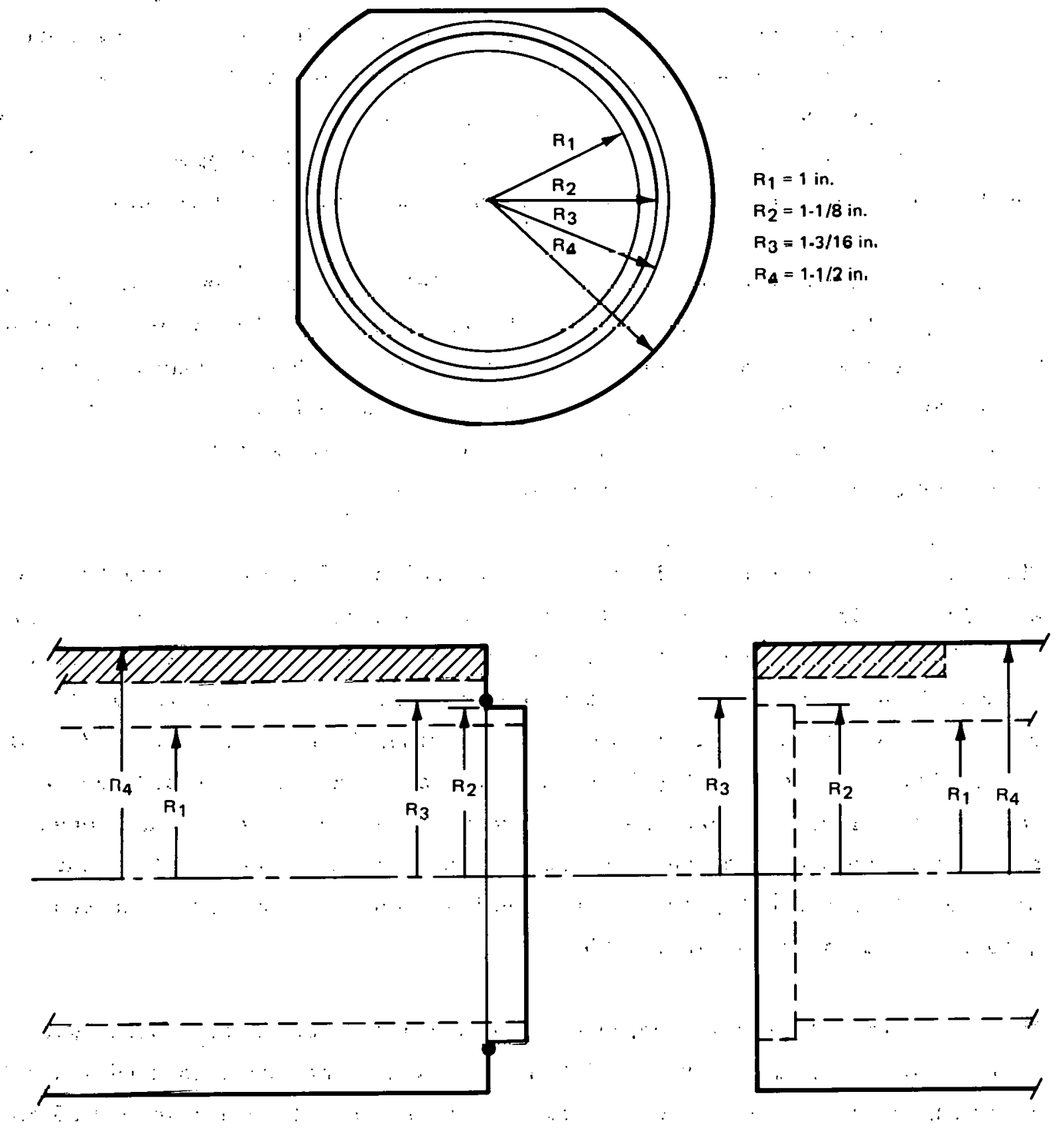

Figure-2-3. End "View and Side View of the Tongue and Groove Arrangement Used to Join the Test Section While Allowing Angular Rotation 


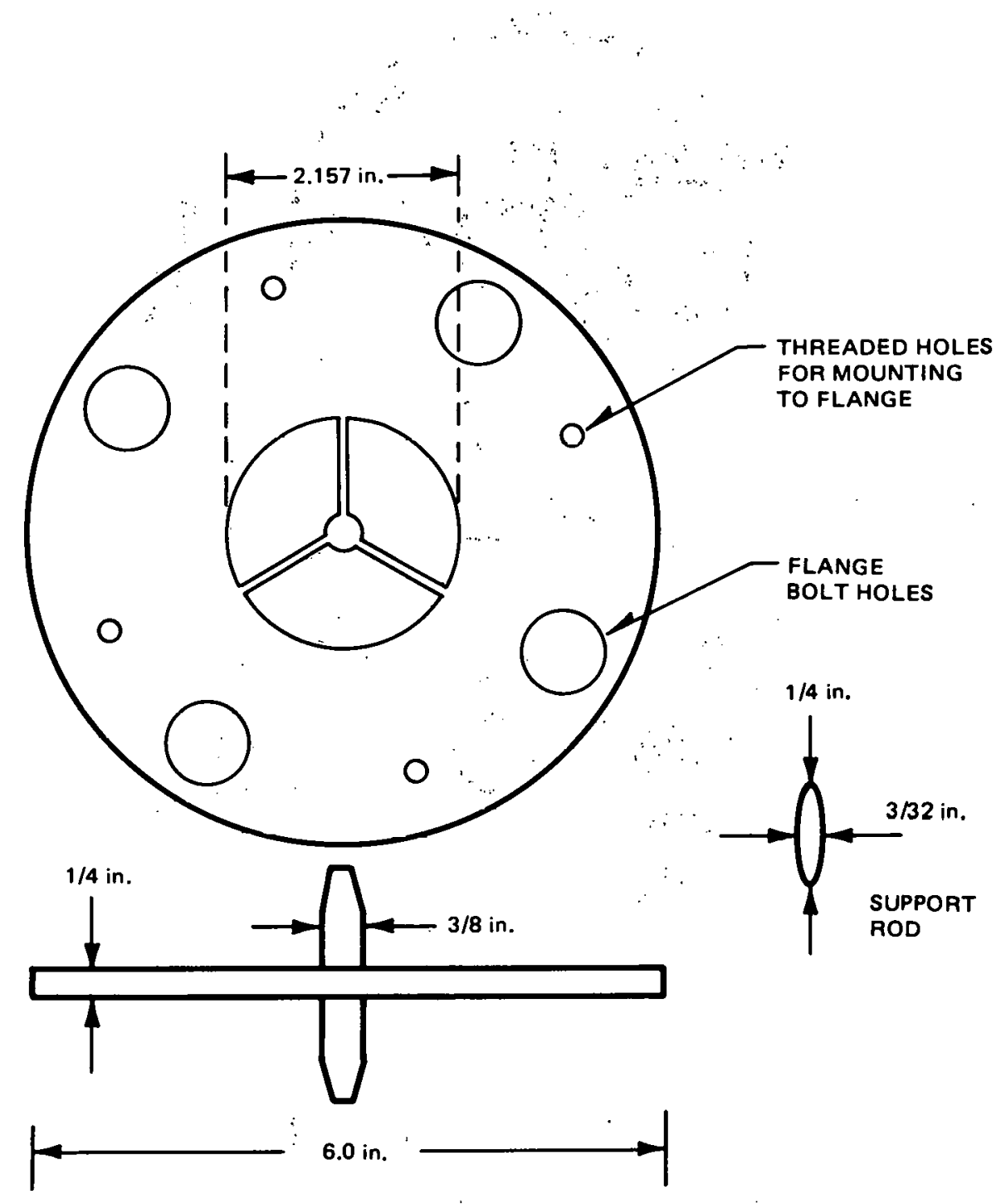

Figure 2-4. Spider Arrangement Used to Align and Support the 1/2-in. Diameter Center Rod at the Flanges 


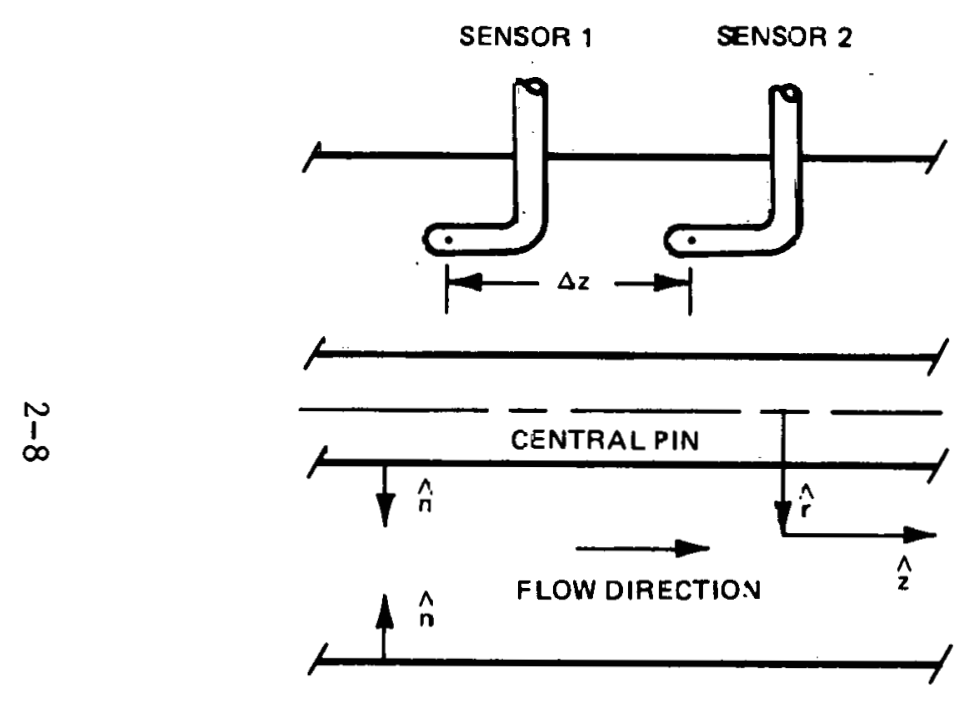

\section{AXIAL}

SEPARATION

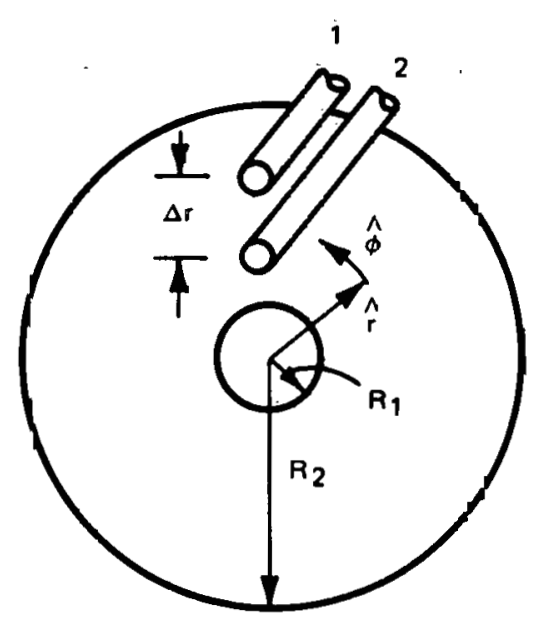

RADIAL SEPARATION
$R^{*}=-R_{1} / /\left(R_{2}-R_{1}\right)$

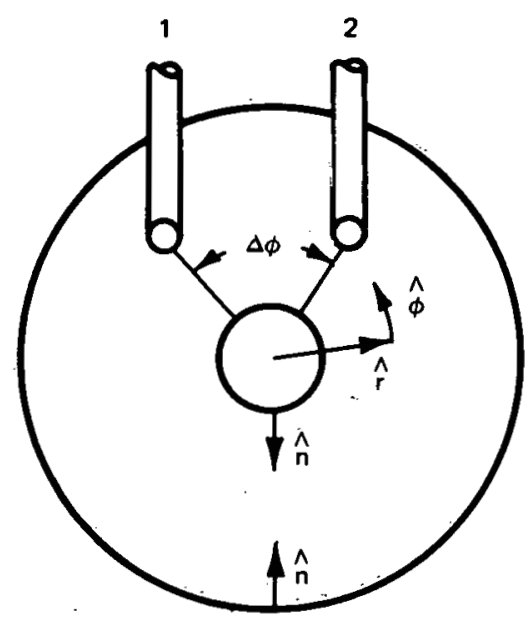

AZIMUTHAL SEPARATION

Figure 2-5. Gecmetry Orientation in the Test Section as it was Defined for This Study, Including the Three Orthogonal Separations of the Transducers 
power curve. The resulting relationship of the mean velocity, $\bar{U}$ (ft/sec), to pressure drop, $\Delta \mathrm{P}$ (in. $\mathrm{H}_{2} \mathrm{O}$ ) is $\overline{\mathrm{U}}=45.23 \Delta \mathrm{P}^{0.55} \mathrm{ft} / \mathrm{sec}$ with a standard deviation of $4.4 \mathrm{ft} / \mathrm{sec}$. This orifice plate calibration was used to monitor the mean velocity in all of the experimental measurements.

\subsection{WALL PRESSURE TRANSDUCERS}

The fluctuating static wall pressure was measured with Celesco Industries, Inc. model LC-70 flush mounting pressure transducers (serial numbers 733, 734 and 750). This pressure transducer model was selected for its high sensitivity (nominally 0.15 volts/psị) and small sensitive area (0.034 sq in.). Details of the transducer dimensions and nominal specifications are given in Figure 2-6.

The physical size of the sensitive ared limits the frequency response of the transducer. Eddies or pressure fluctuations which have a half wave length which is less than the diameter of the transducer can not be resolved properly. For the LC-70 transducer and a mean flow of $200 \mathrm{ft} / \mathrm{sec}$, this wave length corresponds to a frequency of approximately $6.0 \mathrm{KHz}$. A recent study by Bull and Thomas ${ }^{48}$ using pinholes and cavities to couple the wall pressure to the sensor shows that a reduction of resolution also occurs for $\omega \nu / u_{\tau}{ }_{\tau}$ greater than 0.1 where- $\omega$ is the radian frequency, $\nu$ the kinematic viscosity and $u_{\tau}$ the friction velocity. For the LC-70 transducer mounted in the inner wall with a mean flow velocity of $200 \mathrm{ft} / \mathrm{sec}$, this limitation corresponds to $6.4 \mathrm{KHz}$. Since this investigation was primarily centered on the large scale structure in the flow field beyond the boundary layer, it was felt that this transducer gave an adequate balance between frequency response and sensitivity.

The three wall pressure transducers were mounted at the same axial position as shown in Figure 2-7 (position 1 in Figure 2-2). Two of these sensors (nos. 734 and 750) wexe mnunted flush on the outer wall with 180-degree rotation with respect to each other. These were screwed into threaded ports until they were flush with the wall surface and then held in place with a thin lock nut on the back side. 


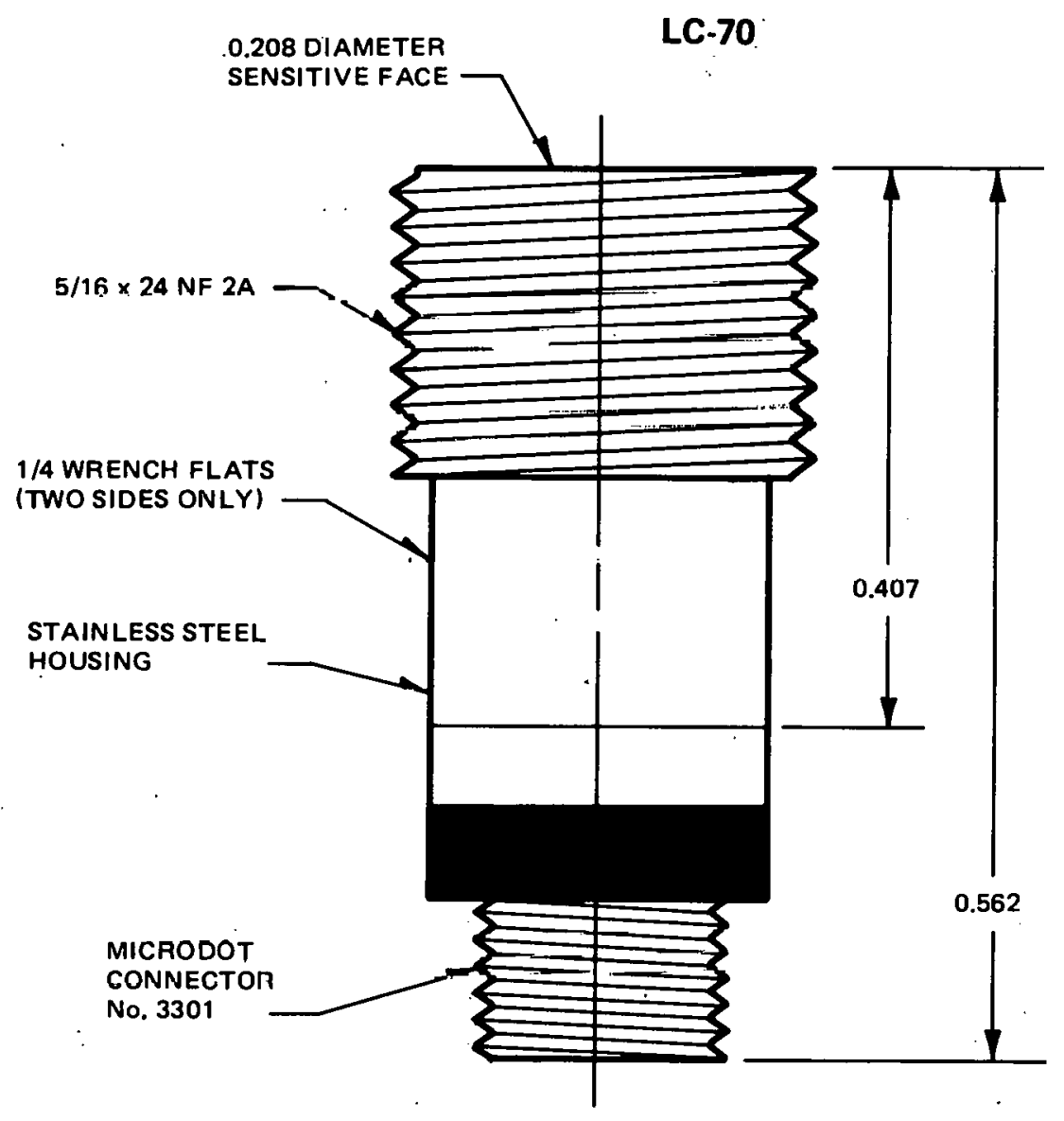

Figure 2-6. Detailed Drawing of the Wall Pressure Transducer as Given by the Manufacturer, Celesco Industries, Inc. 


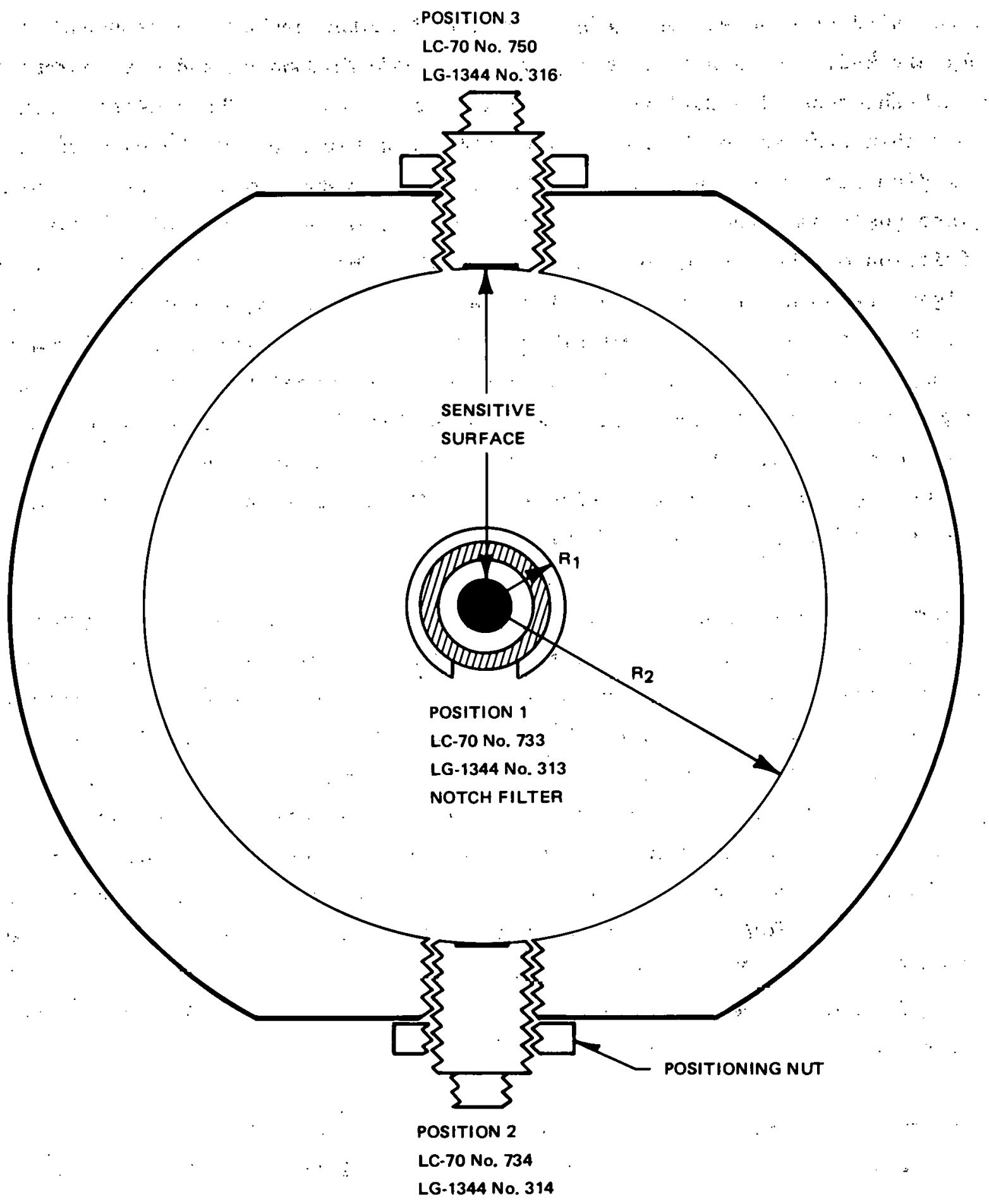

Figure 2-7. End View of Test Section Showing the Individual Wall Pressure: Transducers as Mounted in the Test Section and the Designated Electronics Associated With Each 
The third transducer (no. 733) was mounted in the center rod as shown in Figure 2-8. The sensor is too large to be flush mounted and was thus mounted with the sensitive surface perpendicular to the flow. The sensitive surface was then coupled to the wall by a cavity. The opening in the wall was $0.187 \mathrm{in}$. in diameter. The brass plug in which this transducer was mounted was slid into position through the end of the center rod and held in place by the friction of the 0 -rings on the inner wall of the center rod. The electrical signal from the transducer was carried to the preamp by a low capacitance coaxial cable with (No. 3301) microdot connectors. This cable ran down the inside of the center rod and exited at the downstream spider on the test section: The cable was brought across the flow channel on one of the center rod supports and through the flange. Al1 three transducer signals were input to Celcoco Intistlies, Inc., model LG-1344 high impedance, $40 \mathrm{~dB}$ gain amplifiers (seral numbers 313,314 and 316 ).

A detailed investigation of the wall pressure transducer frequency response was carried out. Each transducer was calibrated against a B \& $\mathrm{K} 1 / 4-\mathrm{in}$. microphone. The calibration procedure was to generate a single frequency sinusoidal acoust1c pressure field. A Wavetek sine wave generator signal was fed into a Bogen amplifier. This amplifier drove a speaker which created a fluctuating sinusoidal pressure field of the same frequency as the Wavetek frequency generator and with an RMS lcvel proportional to the amplifier output. A block diagram of this calibration procedure is shown in Figure 2-9. 'The speakers used for this calibration gave a frequency calibration bandwidth from $40 \mathrm{~Hz}$ to $10 \mathrm{kHz}$. The calibration was carried out in an anechoic chamber to eliminate extraneous acoustic pressure waves and to eliminate reflection of the source waves from the walls.

The direct calibration of the wall pressure transducers is shown in Figure 2-10. All three transducers have very flat frequency responses over the calibration range. The sensitivities, including the $40 \mathrm{~dB}$ gain amplifiers, were 0.384 volts/in. $\mathrm{H}_{2} \mathrm{O}$ for no. $733,0.387$ volts/in. $\mathrm{H}_{2} \mathrm{O}$ for no. 734 and 0.409 volts/in. $\mathrm{H}_{2} \mathrm{O}$ for no. 750. Transducer-amplifier pairings are shown in Figure 2-7. These sensitivities were slightly below the manufacturer's nominal figure of 0.54 volts/in. $\mathrm{H}_{2} \mathrm{O}$. The linearity of the transducers was 


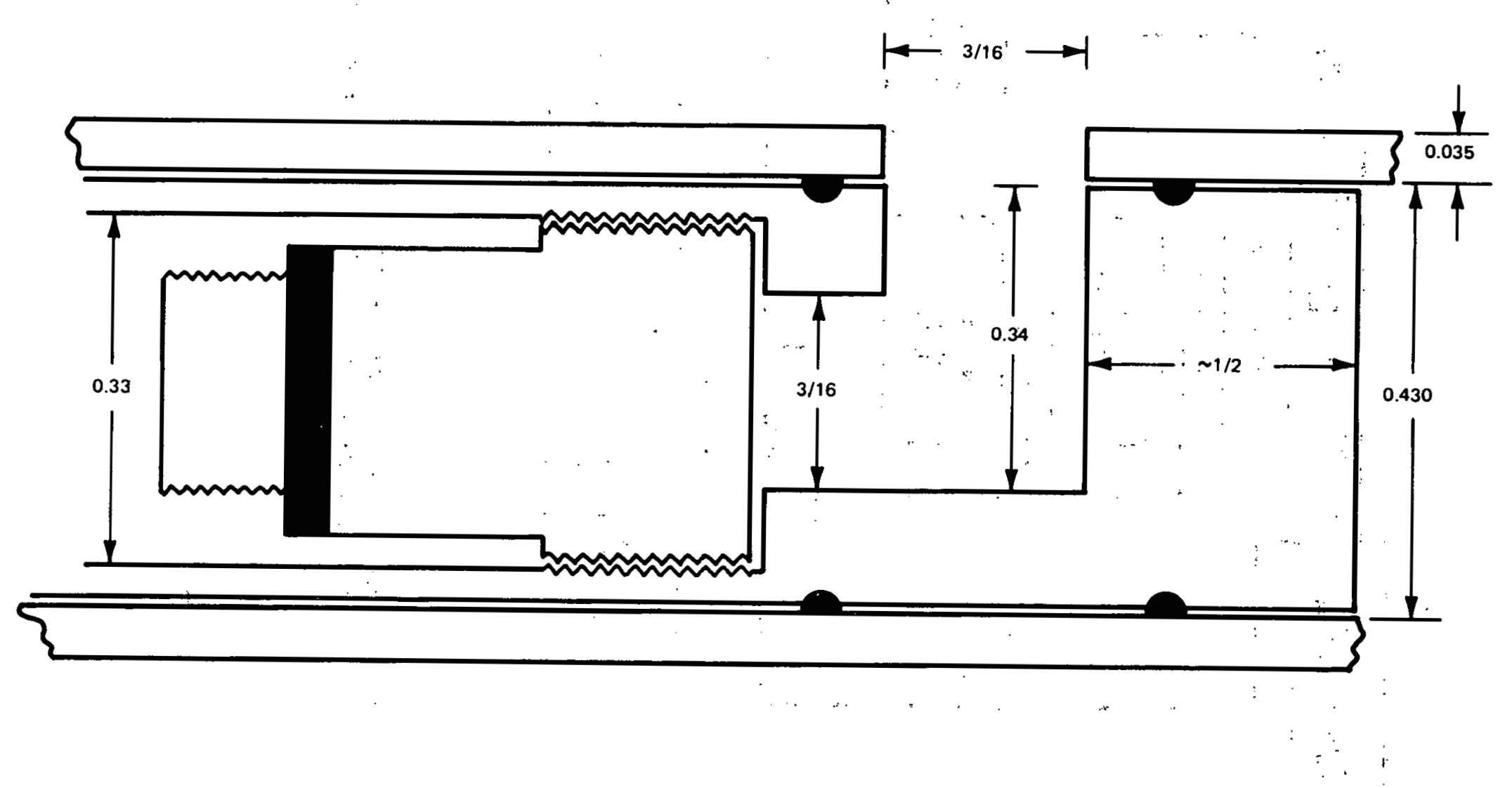

Figure 2-8. Detailed Diagram of the Mounting of the Wall Pressure Transducer in the Center Rod. The Cavity was Filled With Porous Foam 


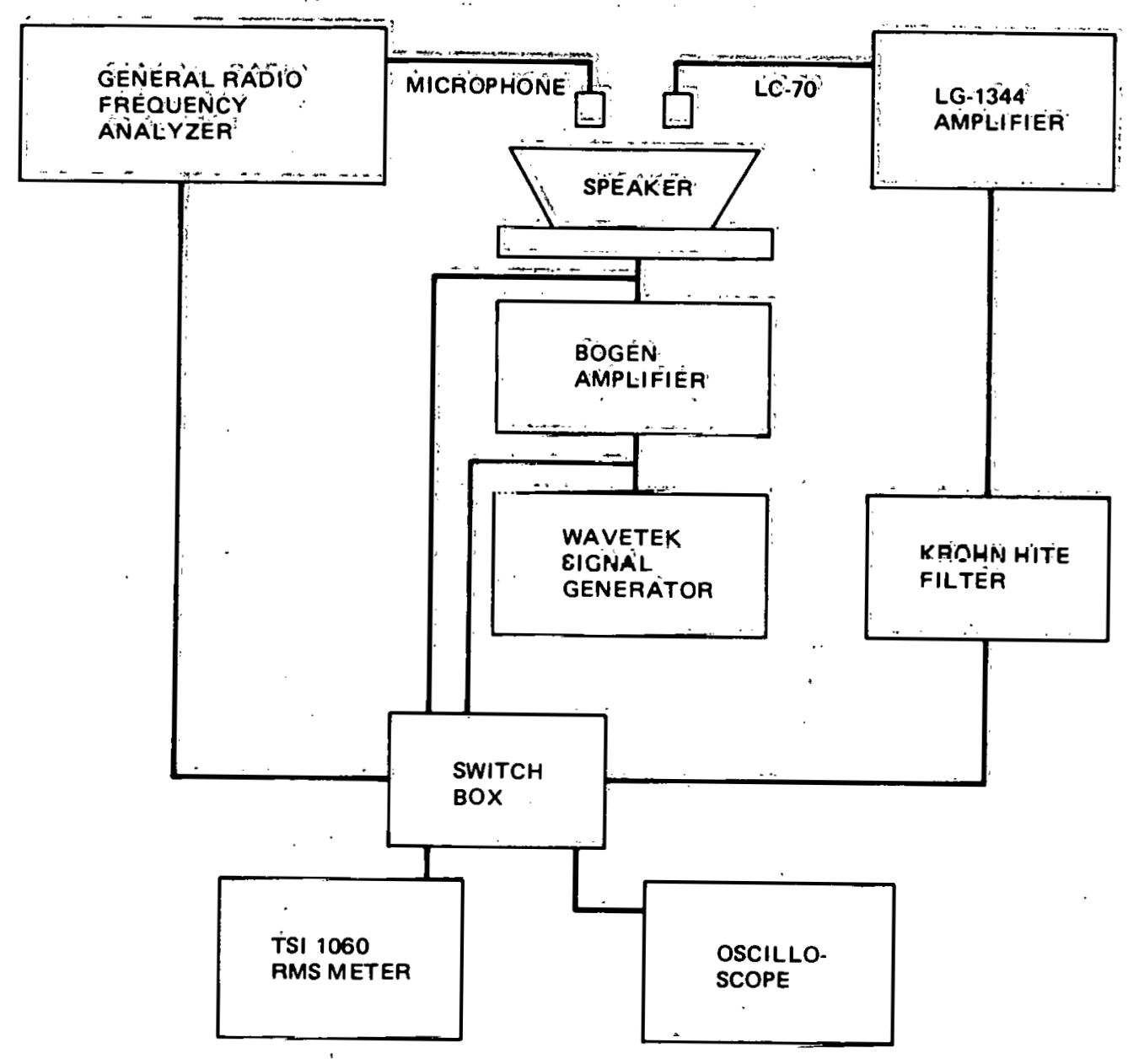

Figure 2-9. Block Dịagram of the Electronics and Tnstrumentation Used in the Wall Pressure Transducer Frequency Response Calibration 


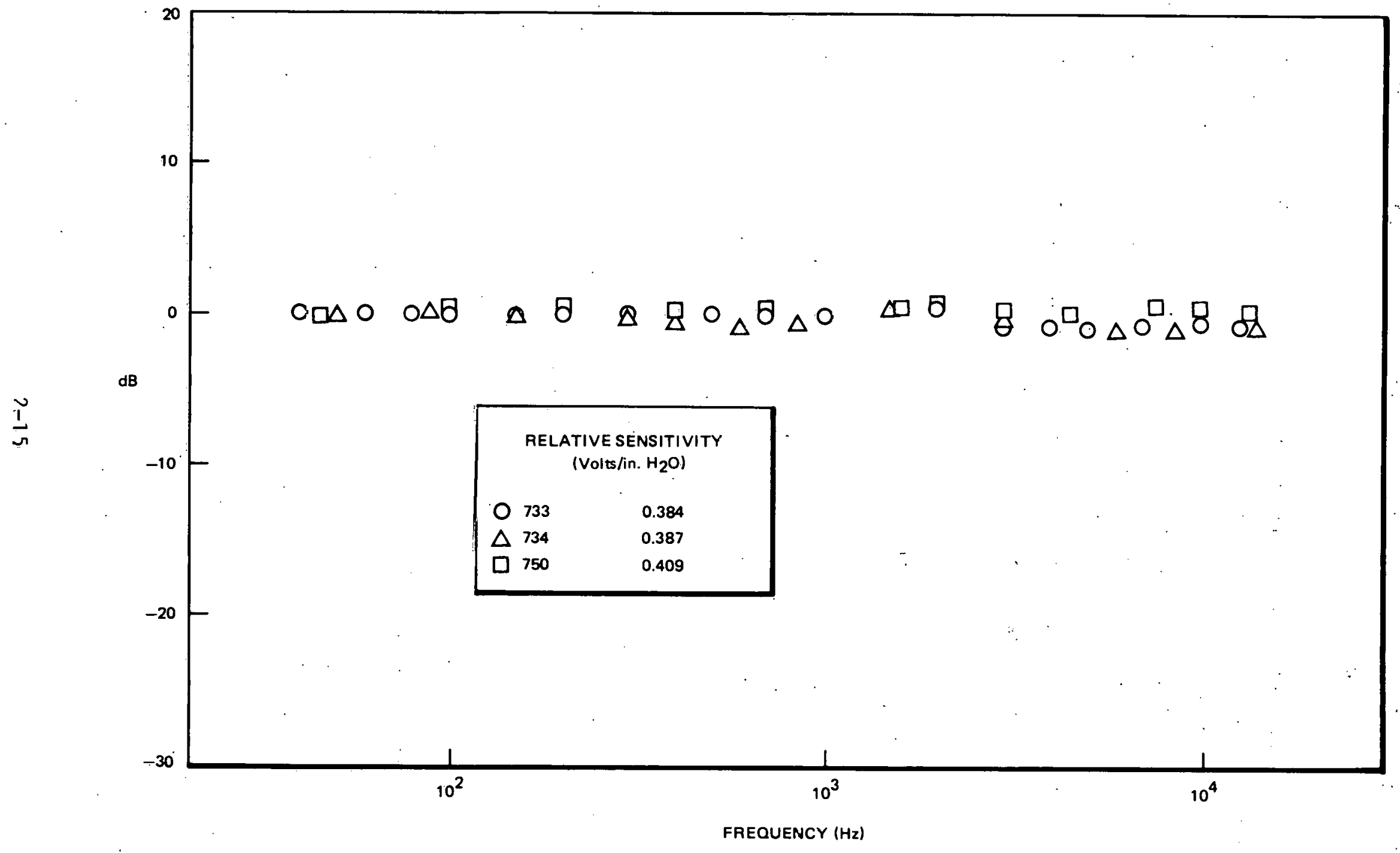

Figure 2-10. Frequency Response of the Wall Pressure Transducers 
checked at several different frequencies with a typical result shown in Figure 2-11 for sensor no. 733 .

This calibration procedure was adequate for the wall pressure transducers which were mounted flush on the outer wall of the flow channel. The inner wall pressure transducer mounting, as noted in Figure 2-8, is geometrically quite different than the outer wall pressure transducer mounting. The cavity coupling the wall pressure fluctuations to the sensitive surface of the pressure transducer will act as a Helmholtz resonating cavity. This resonant frequency can be estimated from the classical relationship for the resonant frequency at." a Helmholtz resonating cavity ${ }^{121}$

$$
f=\frac{e}{2} \sqrt{\frac{T_{11}}{V\left(1_{n}+0.48 r\right)}}
$$

where, $f$ is the resonant frequency, $c$ the sonic velocity of the fluid, $F_{n}$ the area of aperture of the neck, $V$ the volume, $r$ the radius of the neck and $1_{n}$ the length of the neck. Equation (2-1) gives an estimated resonant frequency of $6.5 \mathrm{KHz}$.

The cavity is also capable of being driven at its resonant frequency by a flow past the opening. This single frequency acoustic noise generation is similai lu that obtained by blowing across the open neck of a pop bottle. In order to eliminate this source of acoustic noise, which was observed to dominate the pressure signal, the cavity was filled with porous foan. The toam was fitted to the cavity to give a flow surface tlal was flush with the wall and as smooth as possible. The foam-filled cavity could nnt be acoustically driven by flow over the surface. This arrangement was then calibrated as before. "The resultant frequency response is shown in Figure 2-12, with a sharp resonance at $6.8 \mathrm{KHz}$.

Since this resonance frequency of $6.8 \mathrm{KHz}$ could not be shifted to a higher frequency due to the geometry restrictions on the inner wall pressure transducer, a notch filter was used to reduce the magnitude of the resonance. The filter used was designed by James Bainter of Motorola Semiconductor Products Inc. ${ }^{122}$ The circuit is shown in Figure 2-13. This filter has the advantage 


\section{THIS PAGE}

\section{WAS INTENTIONALLY \\ LEFT BLANK}




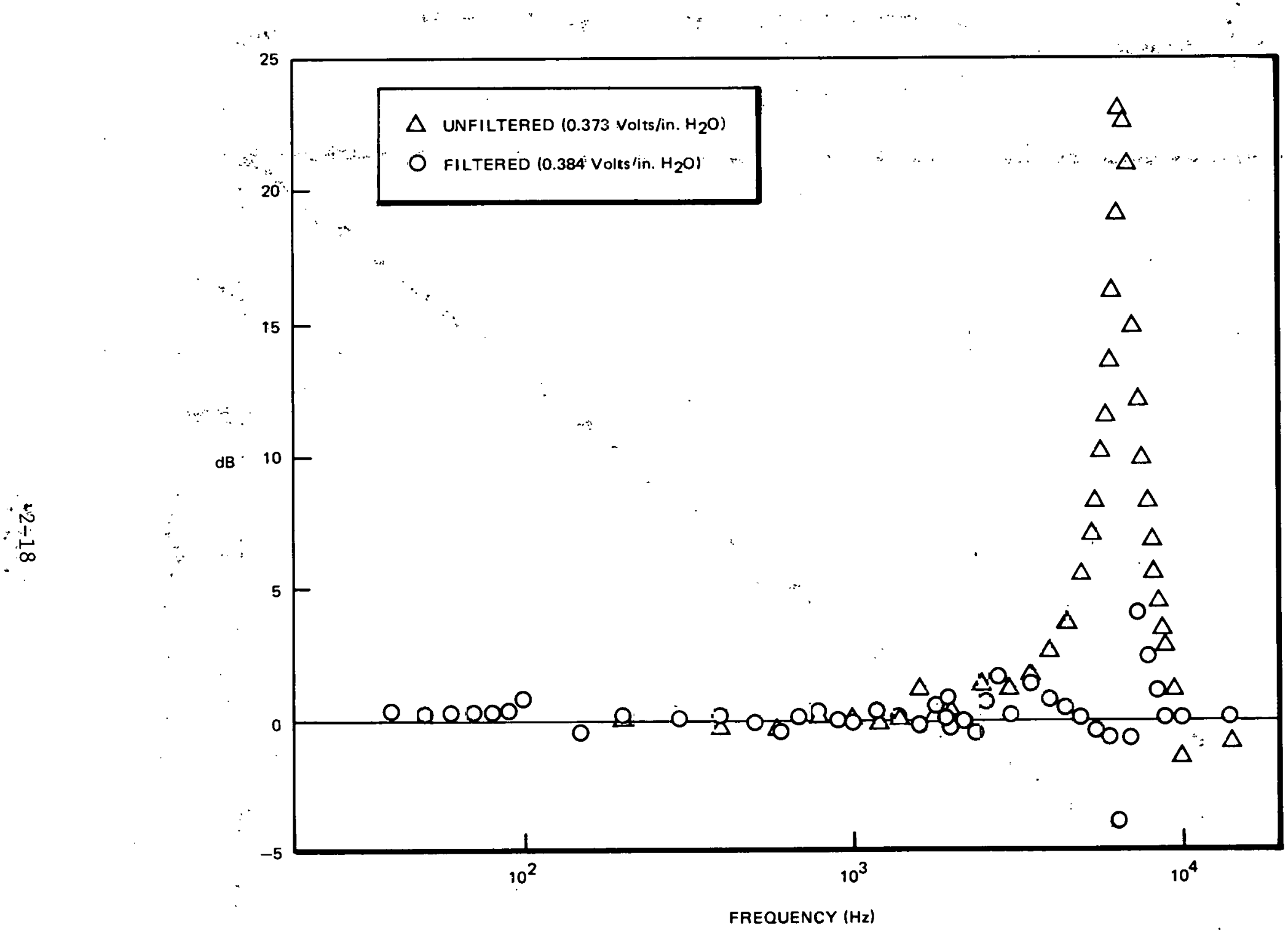

Figure 2-12. Frequency Response of the Inner Wail Pressure Transducer Mounted in the Center Rod With and Without the Notch Bilter 


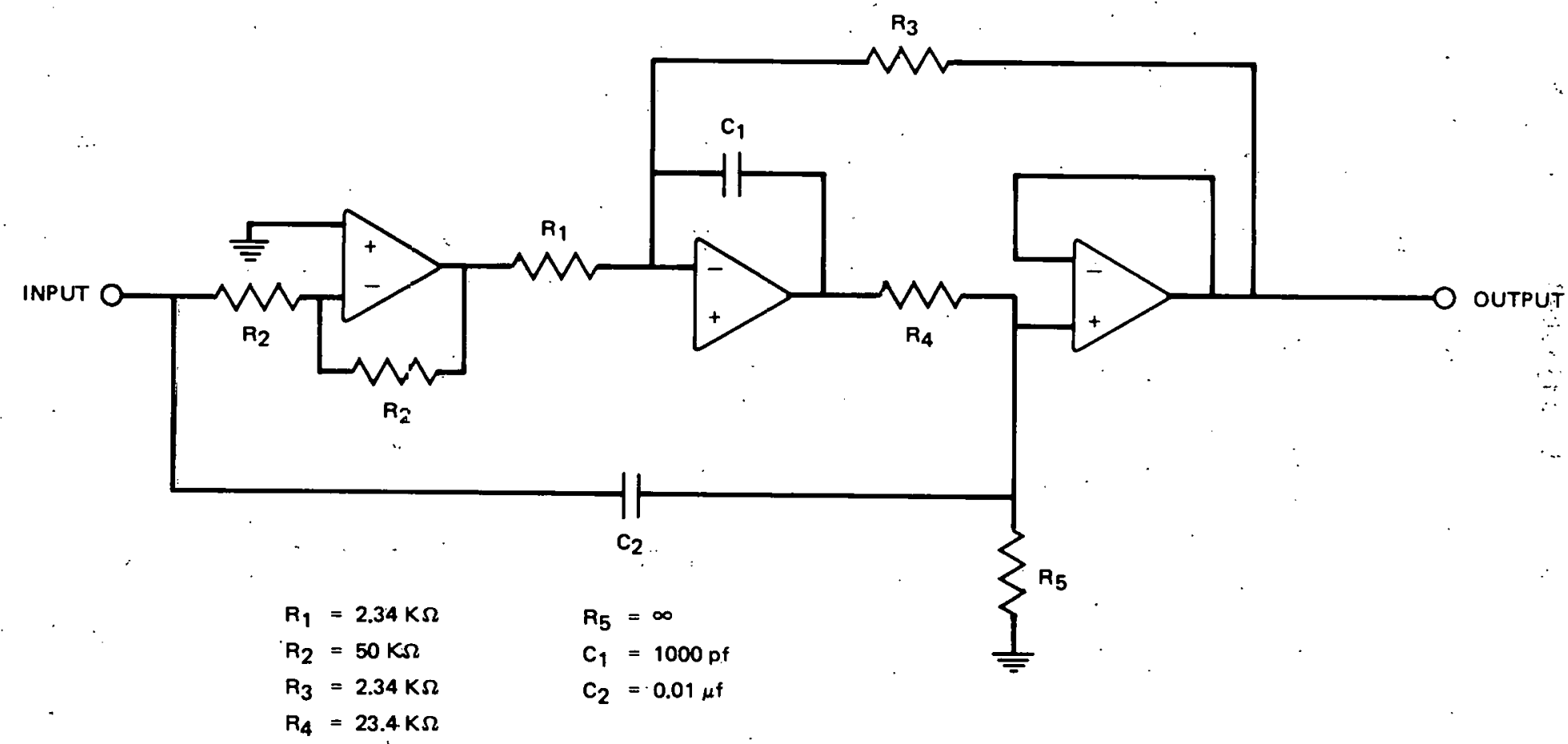

Figure 2-13. Desigr: of the Notch Filter Used to Flatten the Frequency Response of the Inner Wall Pressure Transducer 
of maintaining a constant notch depth, even if resistors and capacitors drift. The notch frequency can be finely adjusted with variable resistors.

The inner wall pressure transducer was then recalibrated with the cavity filled with foam and the notch filter included. This frequency response is also shown in Figure 2-12. The resultant frequency response was relatively flat over the calibration frequency range for the inner wall pressure transducer with a sensitivity of 0.384 volts/in. $\mathrm{H}_{2} 0$. The outer wall pressure transducers exhibit a flat frequency response over the calibration frequency range (Figure 2-10). Thier calibration sensitivities of 0.387 vnlts/in. $\mathrm{H}_{2} 0$ for no. 734 and 0.409 volts/in. $\mathrm{H}_{2} \mathrm{O}$ for no, 750 were recherked with the acnsors in-situ by placing the speaker at the open end of the flow channel and comm paring the relative outputs. of each transducer and found to be unchanged.

The phase angle response between each of the wall pressure transducers was also checked during the in-situ calibration. The phase relationship was less than 10 degrees at all frequencies for all three sensors with the exception of the inner wall pressure transducer at the resonance frequency. This transducer signal was 180 degrees out of phase with respect to the two outer wall pressure transducers at the resonant frequency of $6.8 \mathrm{KHz}$. The phase shift was observable over the frequency range of: the resonance peak, which is about $2 \mathrm{KHz}$ as shown in the response calibration of Figure 2-12.

The location of the wall pressure transducers is important to the data analysis methods which are outlined in Section 3. They are plared at positione, which according to Schumann!s data ${ }^{71}$, are hydrodynamically uncorrelated. This allows the local pressure statistics to be obtained when a significant acoustic pressure contamination is present. This data analysis method is outlined in Section 3 .

Matched phase response is also important in the data analysis as signal subtraction and correlation techniques are used. If there is a difference in phase between two transducers there will be an error introduced when the two signals are subtracted. A 10-degree phase shift represents an error of about $8 \%$; however, the phase shift is much less than 10 degrees with the 
exception of the small frequency range that the resonance peak of the inner wall pressure transducer covers.

The phase and frequency response of the wall pressure transducers is adequate for the data analysis outlined in Section 3. Each of the wall pressure transducers has a very flat response over the frequency range tested (40 $\mathrm{Hz}$ to $10 \mathrm{KHz})$. This frequency range is sufficient for the investigation of the core flow effects of the wall pressure as these effects would be predominantly larger scale, lower frequency fluctuations. The phase matching of the three wall pressure transducers is adequate for the signal subtractions needed to eliminate the acoustic pressure signal which is superimposed on the hydrostatic pressure signal. The improper phase matching from about 6 to $8 \mathrm{KHz}$ will result in an error in the subraction process which will be small as this frequency band is relatively small and the acoustic energy at higher frequencies (above about $400 \mathrm{~Hz}$ ) is much less than it is at the lower frequencies.

\subsection{IN-STREAM BLEED PRESSURE TRANSDUCER}

Measurement of in-stream fluctuating static pressure has long been of interest in experimental research on turbulent flow. The difficulties in obtaining this information have been due to the lack of a suitable sensor. The sensor must be able to measure the static pressure fluctuations while presenting minimal disturbance to the flow. The amplitude frequency response should be flat in the range of interest with a negligible direct response to the fluctuating velocity field or the dynamic head of the mean flow. Finally, the phase lags should be small for simultaneous multi-sensor measurements.

A miniature bleed-type pressure transducer that appears to meet these requirements has been employed to measure the fluctuating static pressure in the flow field region. The structural and design details are laid out by Spencer and Jones ${ }^{123}$. Later modifications by Planchon ${ }^{124}$ included the addition of a static pressure tip to minimize response to velocity fluctuations. Planchon also includes a historical survey of pressure-measuring devices. 
The two pressure transducers used are shown in Figure 2-14. The transducer operates by bleeding air in laminar flow through a capillary tube from a; constant pressure reservoir. Pressure fluctuations at the downstream end of the capillary tube set up fluctuating velocities in the bleed fluid, which., are measured by a 0.001 in. diameter hot-film sensor mounted across the exit of the capillary tube. The bleed fluid flows through the static pressure. tip and exits via six circumferential bleed holes, which are each 0.013 in. in

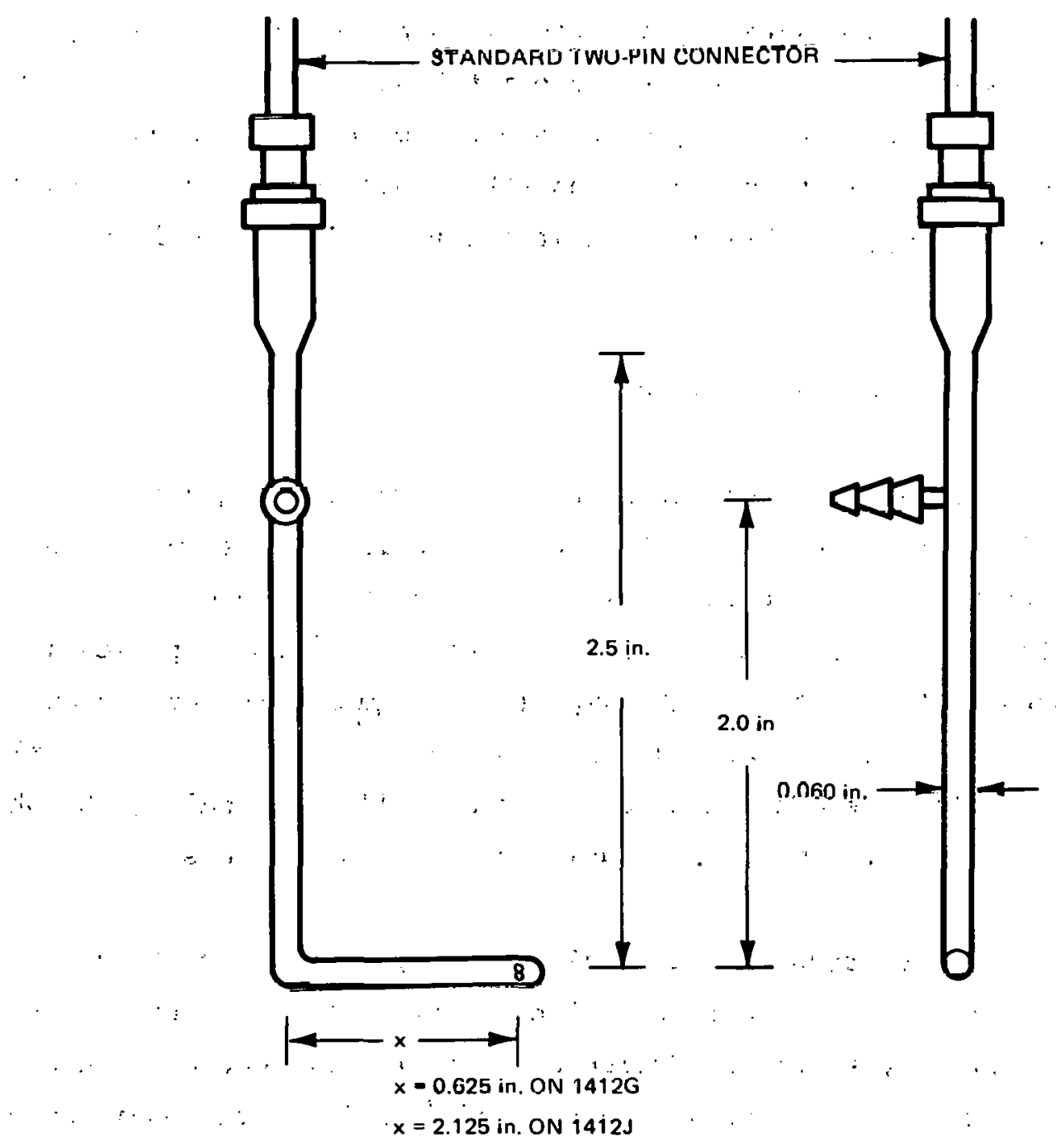

Figure 2-14. Schematic Drawing of the Two Bleed Pressure Transducers With the Essential External Dimensions 
diameter and which are 2.5 diameters downstream of the tip. The hemispherically contoured, highly polished tip presents a minimum disturbance to the flow. The transducer size of $0.060 \mathrm{in}$. in diameter satisfies the requirement that the transducer be smaller than the turbulent space scales of interest as the integral space scales in the radial and azimuthal directions are estimated to be greater than 0.2 in.

The operation of the pressure transducer requires that the bleed fluid, dry bottled air in this case, be injected into the free stream. At the selected operating conditions this volumetric flow rate: is 0.001 cubic:in./sec per bleed hole. Planchon ${ }^{124}$ has shown that at very low free stream velocities (less than $50 \mathrm{ft} / \mathrm{sec}$ ) and high bleed rates ( 0.003 cubic in./sec), the effect of the bleed injection is seen as a thickening of the probe boundary layer which results in a sensitivity to axial velocity fluctuations. However, at free stream velocities above $75 \mathrm{ft} / \mathrm{sec}$ and bleed rates of 0.001 cubic in./sec per bleed hold this: effect is not discernible.

The frequency response of the bleed pressure transducer is fixed by the size of the capillary tube, the hot-film sensor and the bleed fluid properties. These variables are adjusted to give optimum frequency response while maintaining a relatively high sensitivity. The physical characteristics which control the response are matched as closely as possible by the manufacturer, Thermo-Systems, Inc:, to give two pressure transducers with nearly identical frequency responses:

A static sensitivity of the bleed transducers can be obtained by varying the pressure drop across the bleed tube while measuring the d.c. voltage output from the hot-film sensor. The optimum operating conditions were found to be with an overheat ratio of 1.7 and an air bleed back pressure of 6.0 in. $\mathrm{H}_{2} 0$. The voltage output of the pressure transducer is a function of the bleed pressure to the one-half power. However, for low bleed velocities and low pressure fluctuations the output can be assumed to be linear as shown in Figure 2-15. The maximum pressure fluctuation from the mean pressure level was estimated to be less than 1.0 in. $\mathrm{H}_{2} \mathrm{O}$ and was later found to be even less. The data of Figure 2-15 were least squares fitted with a linear function to 


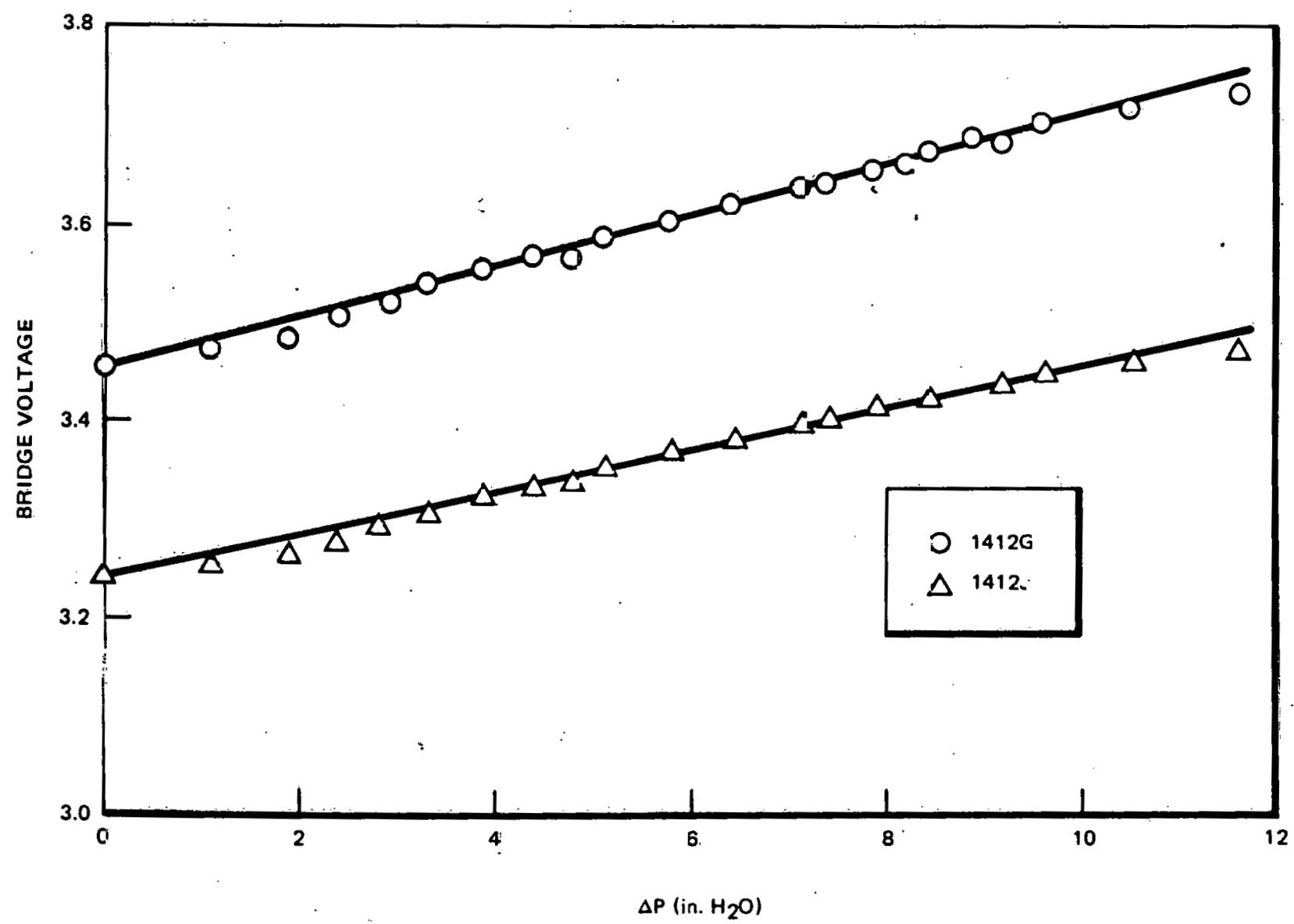

Figure 2-15. Bleed Pressure Transducer Static Calibration Data Yielding the Sensitivity of the Sensors at Low Frequency $\left(25.1 \mathrm{mV} /\right.$ in. $\mathrm{H}_{2} \mathrm{O}$ for $1412 \mathrm{G}$ and $21.4 \mathrm{~m} \nabla / \mathrm{in} . \mathrm{H}_{2} \mathrm{O}$ for $1412 \mathrm{~J}$ ) 
find the sensitivity. The static sensitivity of pressure transducer $1412 \mathrm{G}$ was found to be $25.1 \mathrm{mV} / \mathrm{in}$. $\mathrm{H}_{2} \mathrm{O}$ and that of $1412 \mathrm{~J}$ was $21.4 \mathrm{mV} / \mathrm{in}$. $\mathrm{H}_{2} \mathrm{O}$ at a reservoir pressure of 6.0 in. $\mathrm{H}_{2} \mathrm{O}$ above ambient.

The frequency response was determined by comparing its output to the output of a 1/4-in. capacitance microphone. An acoustic pressure field was generated as outlined in Subsection 2.2. The calibration was carried out in an anechoic. chamber to eliminate reflections and interference. This calibration procedure was identical with that of the wall pressure transducers. The instrumentation block-diagram for this calibration is shown in Figure 2-16. The frequency response of each of the two bleed pressure transducers is shown in Figure 2-17 as the open symbols. The response is non-linear over the frequency range of Interest, 0 to $10 \mathrm{kHz}$. The response was corrected with an RC circuit design shown in Figure 2-18. The resultant frequency response is also shown in Figure 2-17. This corrected response is acceptably flat over the frequency range of interest, and the static sensitivities determined from varying the bleed pressure agree with the dynamic sensitivities determined from the comparison with the microphone. The final sensitivity of the bleed transducers including the $\mathrm{RC}$ correction circuit is $5.2 \mathrm{mV} / \mathrm{in} . \mathrm{H}_{2} \mathrm{O}$ for $1412 \mathrm{G}$ and $4.4 \mathrm{mV} / \mathrm{in}$. $\mathrm{H}_{2} \mathrm{O}$ for $1412 \mathrm{~J}$.

The bleed pressure transducers were mounted on the test section with the instrument holding device shown in Figure 2-19. The device is shown mounted onto the test section with a bleed pressure transducer in place. This allows the preesure transfir.er to be accurately located radially in the channel to $+/-0.001$ in. Rotation of the center section of the test section allows for azimuthal orientation to $+/-1$ degree. Different mounting ports at several axial locations allow the bleed pressure transducer to be mounted at different axial heights. The physical dimensions of the two bleed pressure transducers make it possible to locate one in the rotating section and one in the nonrotating eection while both measure the pressure at the same axial location. This allows azimuthal and radial separation of the bleed pressure transducers. 


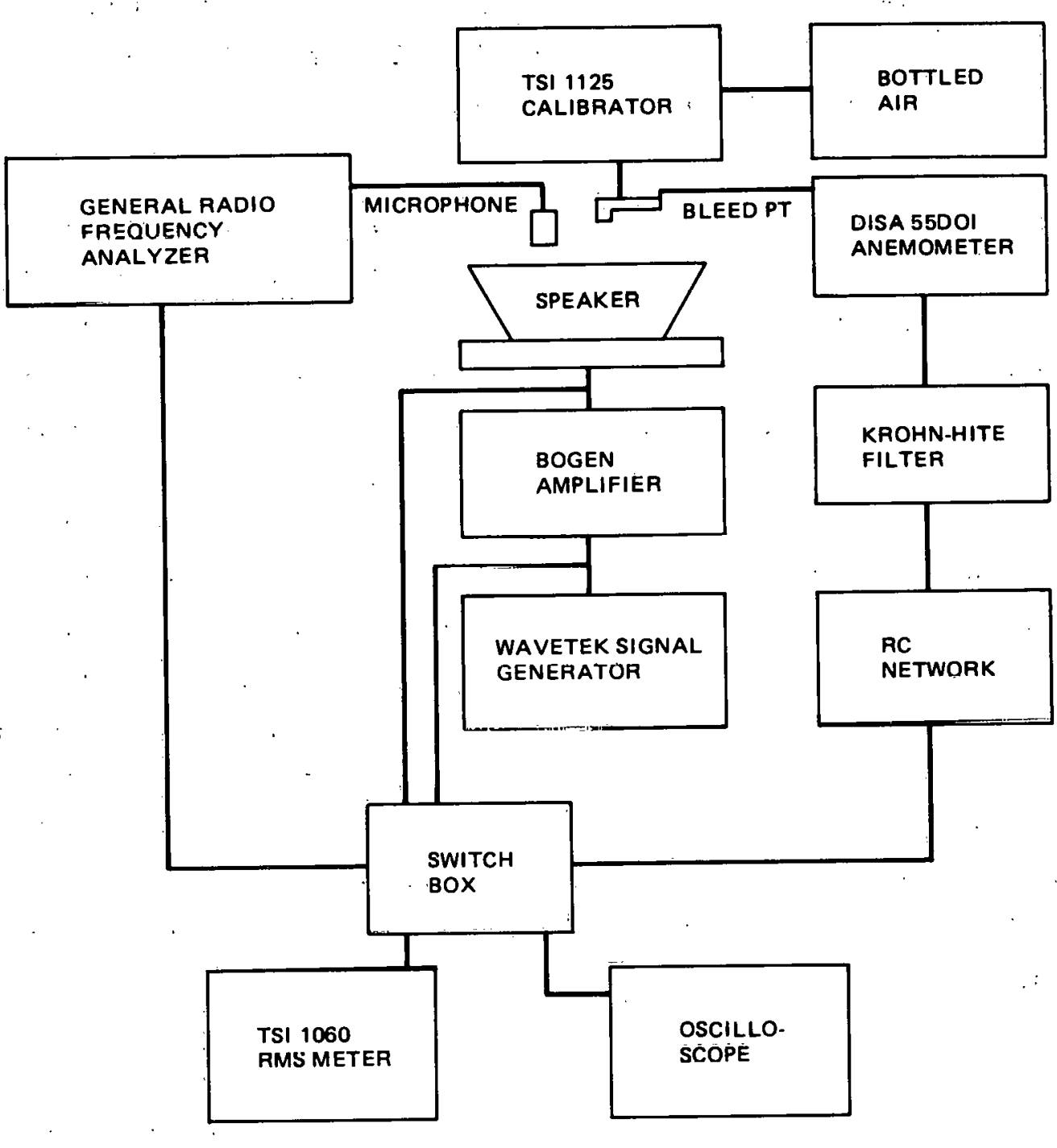

Figure 2-16. Block Diagram of the Electronics and'Instrumentation for the Frequenry Response Galibration of the Blecd Prcsaure Transducers 


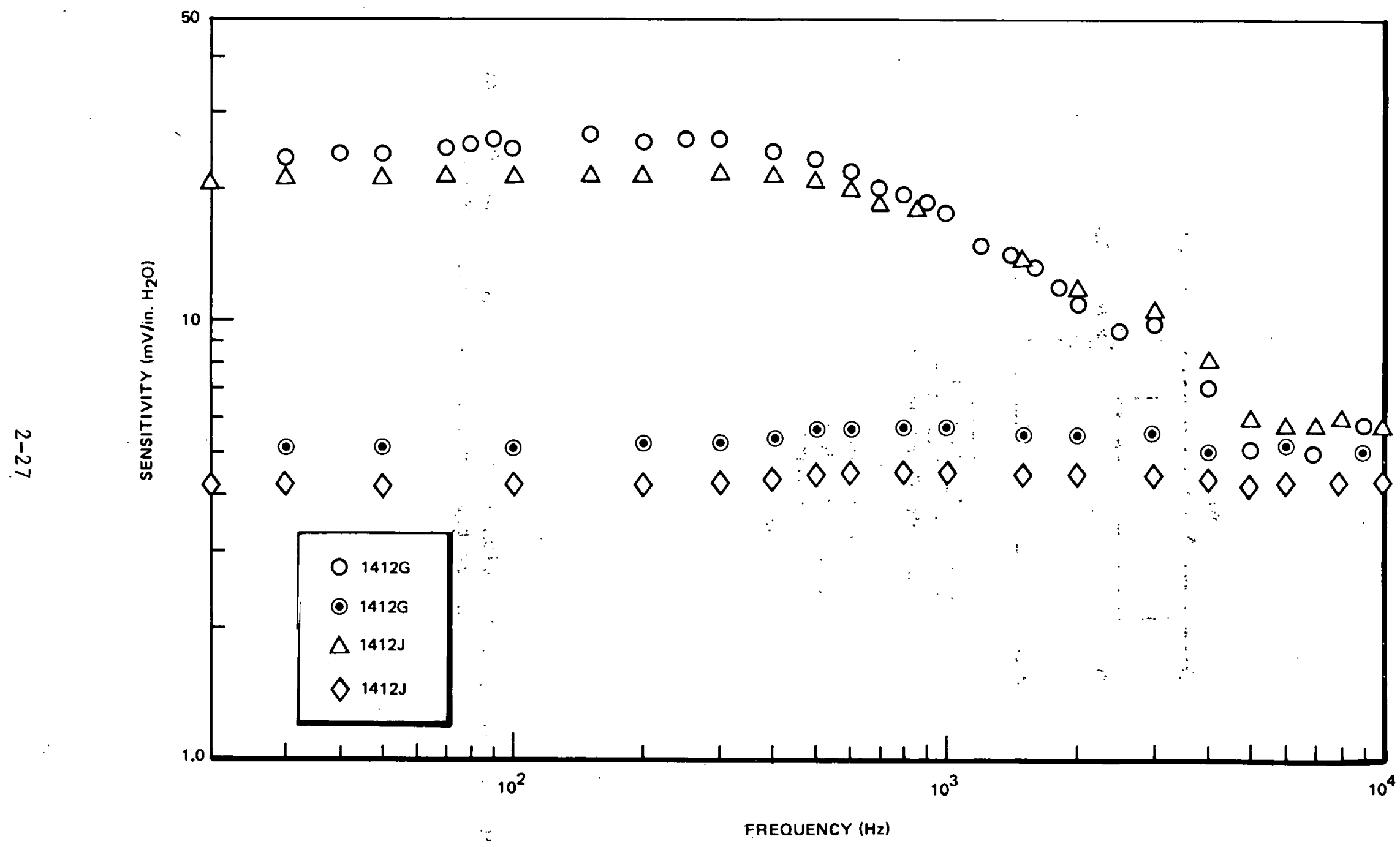

Figure 2-17. Frequency Response of the Bleed Pressure Transducers With and Without the RC Correction Network 


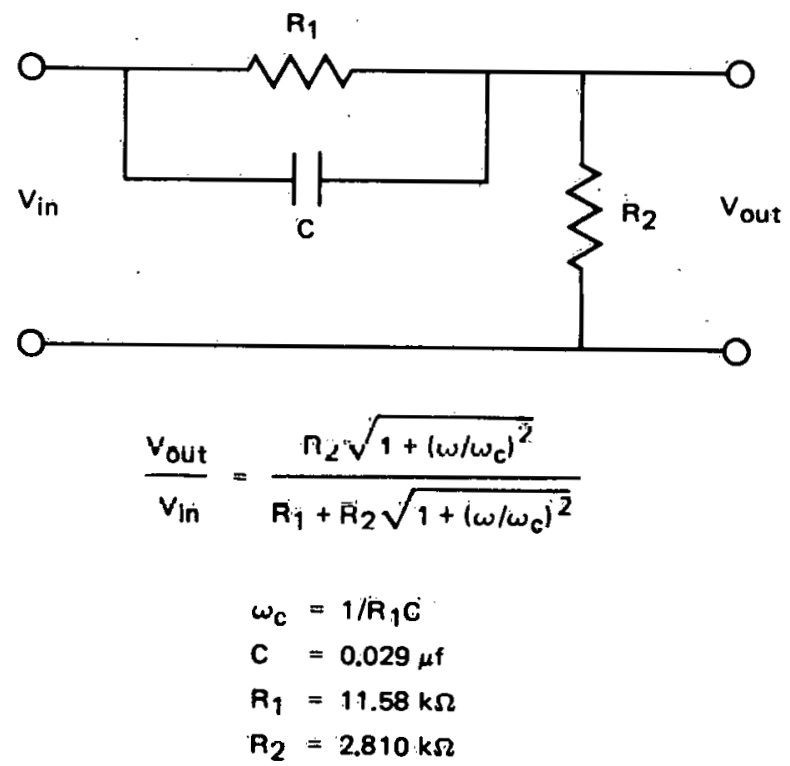

Figure 2-18. RC Correction Network for Flattening the Frequency Responsc of the Bleed Pressure Transducers 


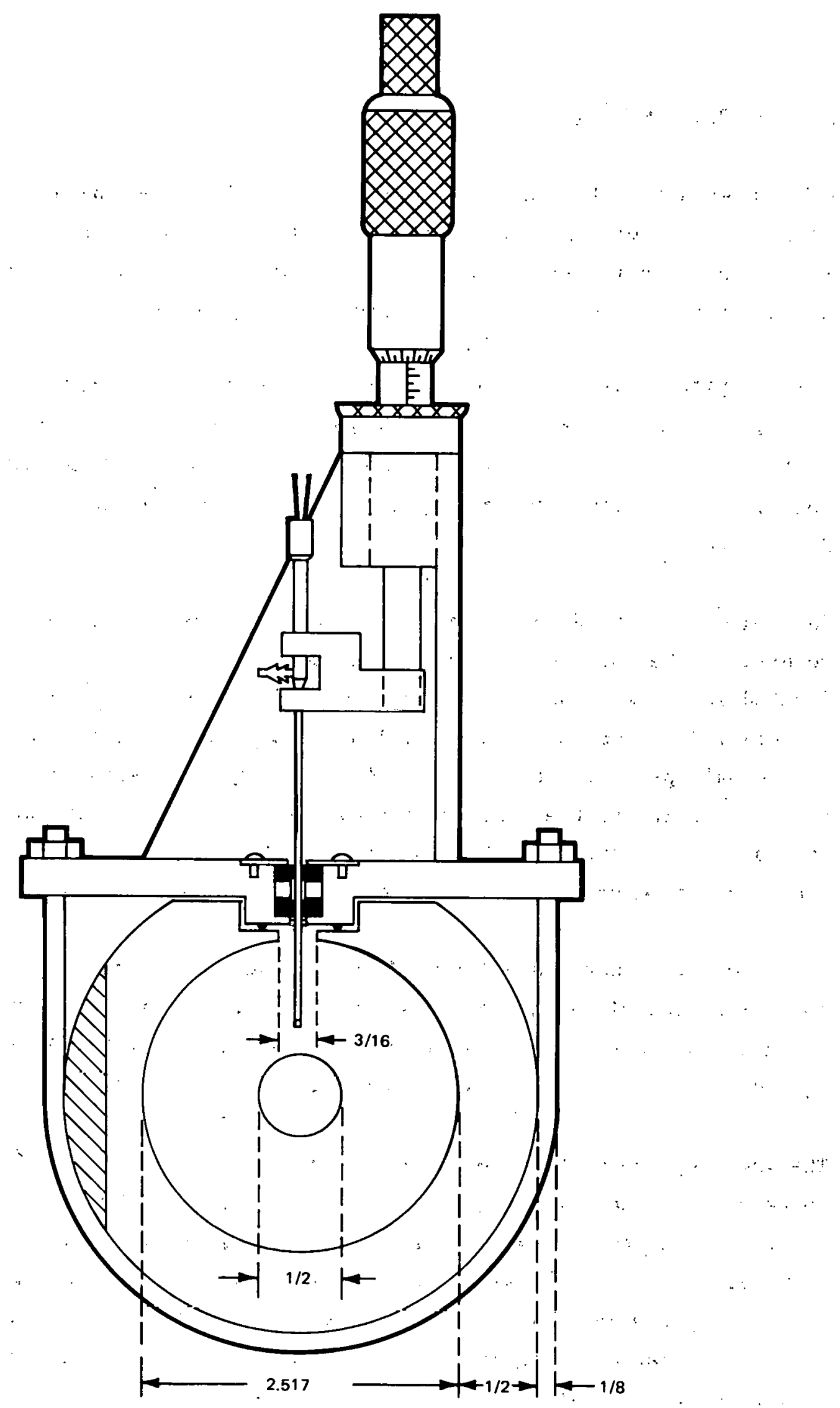

Figure 2-19. Instrument Holder Shown Mounted on the Test Section With a Bleed Pressure Transducer in Place 


\subsection{VELOCITY SENSORS}

A Thermo-Systems, Inc. mode1 $1245 \mathrm{G}-\mathrm{Ti} .5$ cross flow X-probe was used for velocity measurements. The orientation of the $\mathrm{X}$-probe was such that the axial and radial velocity fluctuations could be measured. The $X$-probe was designed to fit into the instrument holder shown in Figure 2-19.

The 0.00015-in. diameter tungsten platinum coated hot-wires were operated at a constant temperature using DISA anemometers and linearizers and an overheat ratio of 1.7 . The sensor length-to-diameter ratio of 333 satisfies the requirement for sine law cooling established by Champagne ${ }^{125}$ and 1 outlined in Section 3.

The sensor, inserted in the instrument holder shown in Figure 2-19 was mounted on the rotating section of the test section. The hot-wires were located in the same axial plane as the wall pressure transducers. This allowed radial and azimuthal location of the velocity sensor with respect to the wall pressure transducers. Positioning the instrument holder in different axial ports on the test section allowed axial separation of the wall pressure transducers and the' $\mathrm{X}$-probe. . In the current test section the $\mathrm{X}$-probe could only be moved downstream from the wall pressure transducers.

The velocity sensor was calibrated in the low turbulence snre nf a suhsnnir. jet. The linearized anemometer output, as a function of mean fluid velocity is shown in Figure 2-20 from which the sensitivity was found, by a least squares linear fit of the data, to be 0.0305 volt $/ \mathrm{ft} / \mathrm{sec}$.

'The yaw response of the X-probe is shown in Figure 2-21 for which the output voltage of the anemometer (E), normalized to the zero yaw output $\left(E_{o}\right)$, is plotted versus the yaw angle $(\alpha)$. The yaw response is shown to be in very good agreement with the sine law cooling hypothesis outlined in Section 3 for the yaw angle present in the flow system. Since the maximum turbulence intensity in the radial or azimuthal directions is about $8 \%$, the corresponding instantaneous velocity vector of a three standard deviation fluctuation will yield only a 15-degree angle: to the mean velocity. Thus, the velocity vector will be within $+/-15$ degrees of the mean flow velocity vector about $98 \%$ of the time. 


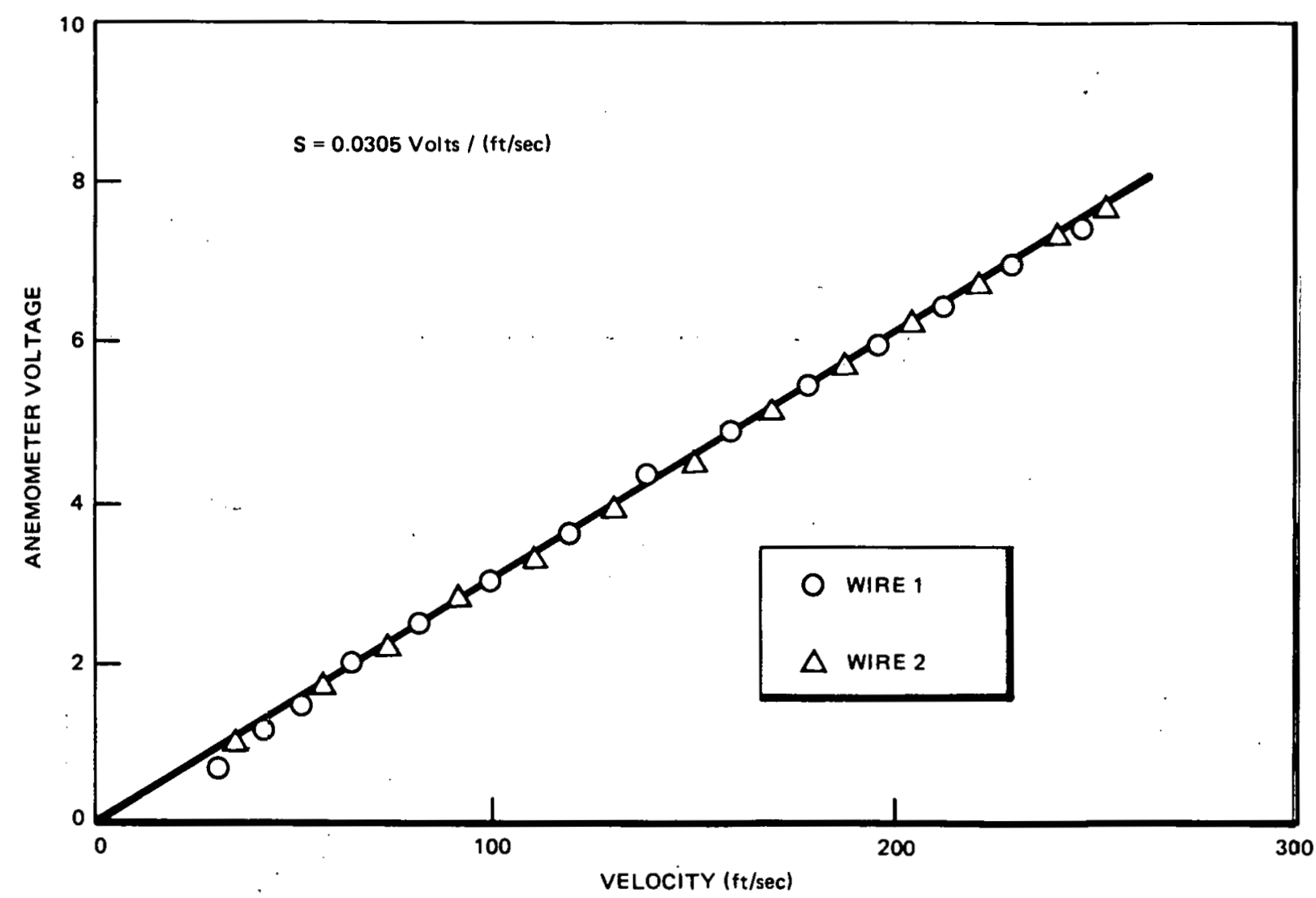

Figure 2-20. Linearized Calibration Curve of X-Probe 1249G-T1.5 


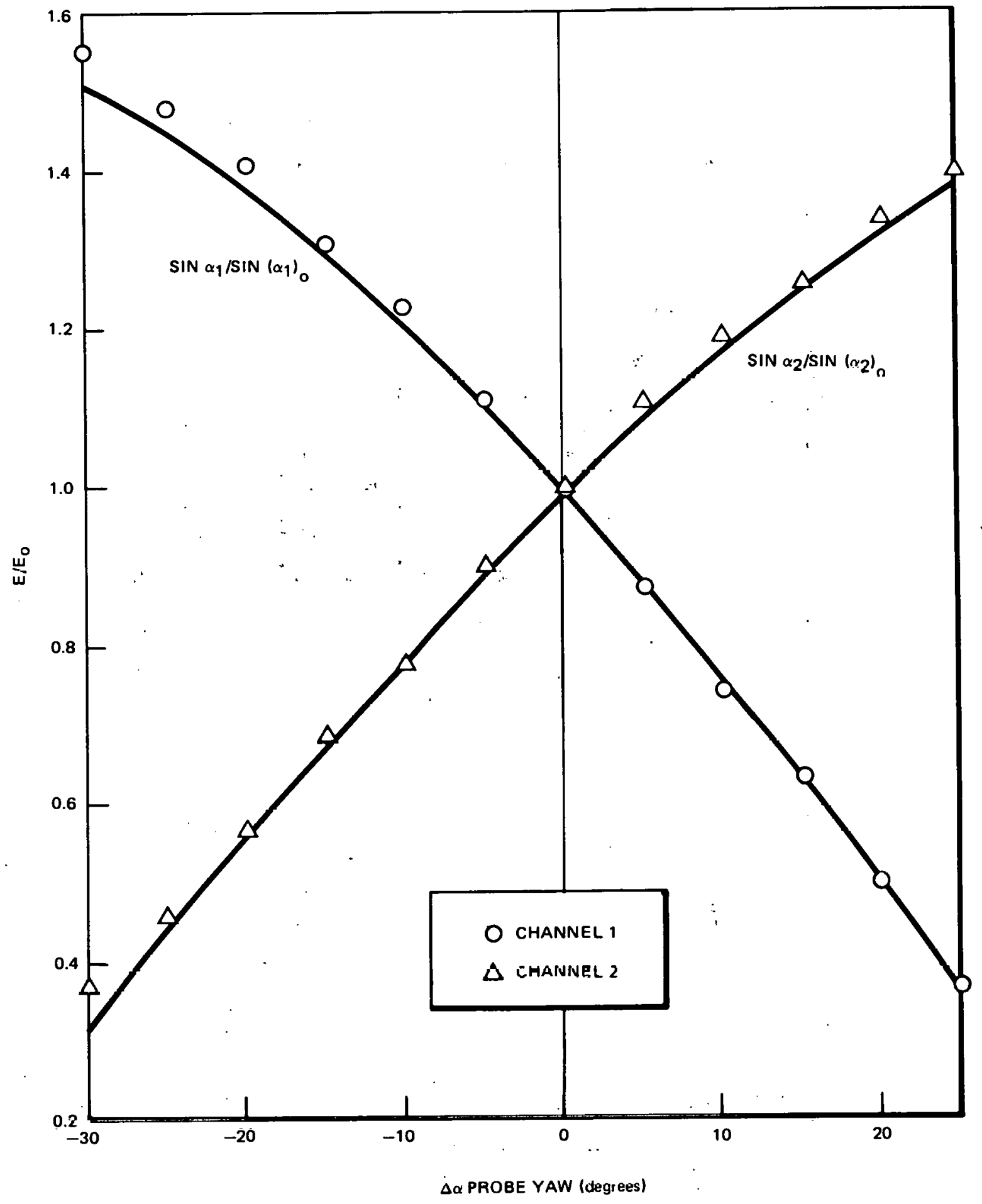

Figure 2-21. Yaw Response of X-Probe 1249G-T1.5 With $\left(\alpha_{1}\right)_{0}=38^{\circ}$ and $\left(\alpha_{2}\right)_{0}=42^{\circ}$ for a Relative Angle of $40^{\circ}$ Between the Two Wires and the Mean Velocity $\bar{U}$ 
The resultant calibration of the $\mathrm{X}$-probe shows a very linear response to velocity in the velocity range of interest (100 to $300 \mathrm{ft} / \mathrm{sec}$ ). The yaw test verifies the sine law cooling hypothesis and gives the relative angle between the two sensors to be 40 degrees: This angle is necessary to obtain the proper separation of the two velocity components being measured by the $\mathrm{X}$-probe.

\subsection{INSTRUMENTATION}

The wall pressure measurements were made with Celesco Industries, Inc. model LC-70 flush mounting pressure transducers. The output from these transducers was fed to Celesco Industries, Inc. model LG-1344 high impedance, $40 \mathrm{~dB}$ gain amplifiers. This signal was then adequate for either data analysis or signal recording.

The in-stream fluctuating pressure measurements were made with a ThermoSystems Inc. model 1412 bleed-type pressure transducer. This transducer was internally modified and a specially designed static pressure tip was added to minimize response to velocity fluctuations. ${ }^{124}$ These modifications were detailed by Planchon. ${ }^{124}$ A DISA model 55D01 constant temperature anemometer was used to operate the bleed pressure transducer. The bleed fluid was bottled air and the flow to the transducer was regulated with a Thermo-Systems Inc. model 1125 calibrator. The anemometer output was fed into a Krohn-Hite model 3750 filter where it was amplified by a factor of 10 and band passed through a bandwidth of $0.1 \mathrm{~Hz}$ to $20 \mathrm{KHz}$ with $24 \mathrm{~dB} /$ octave filters. The output signal from the filter was adequate for data analysis or signal recording.

The frequency calibration of the pressure transducers was performed with a Bogen "Challenger" amplifier and a University model ID-60T driver unit as the source of a single frequency pressure fluctuation. A Wavetek model 110 signal generator was used as an input to the amplifier. The signal generator was used to control the driver output frequency. The driver pressure field was measured with a Bruel and Kjoer 1/4-in. capacitance microphone which was powered by a General Radio mode1 $1564-\mathrm{A}$ sound and vibration analyzer. 
The velocity measurements were made with a Thermo-Systems Inc. model 1249G-T1.5 cross flow X-probe. DISA model 55D01 constant temperature anemometers were used to operate the two hot-wires. The anemometer output was fed into DISA model 55D10 linearizers. The linearization constants and voltage offset were selected from anemometer bridge output data as described in the unit operating manual. The linearizer output was then adequate for data analysis or signal recording.

A Sangamo model 3564, 14-track, frequency modulated magnetic tape recorder was used to store the on-line signals for later processing and analysis. It

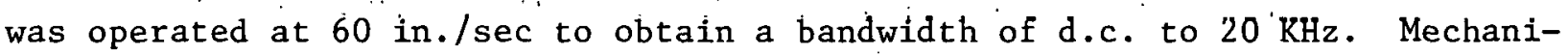
cal difficulties with the tape recorder forced some of the data analysis to be done on-line. In either case the analysis methods and instrumentation were the same.

All d.c. voltages were measured with a Hewlett-Packard model 3465B multimeter. The RMS voltages were measured with a Thermo-Systems Inc. model 1060 true RMS voltmeter. All voltage signals were monitored on a Tektronix model 5112 oscilloscope. A Thermo-Systems Inc. model 1015-C correlator was used with the $\mathrm{X}$-probe to separate the velocity components. This same correlator, or another identical one, was used for some of the zero-time correlations and for the shear stress measurements. Correlations requiring time delays were performed with a 400 point Saicor model SAI-43 digital correlation and probability analyzer. The correlation was output to a Houston Instrument mode1 $2000 \mathrm{X}-\mathrm{Y}$ recorder for analysis. Ihe digital correlätion was alsó fed directly to d Saicor model SAI-470 Fourier transtorm analyzer for spectral analysis. 


\section{DATA ANALYSIS TECHNIQUES}

\subsection{INCLINED HOT-WIRE RESPONSE FOR VELOCITY MEASUREMENTS}

The response of a hot-wire in a flow field is a function of the effective cooling velocity, $\mathrm{U}_{\text {eff }}$. The geometrical orientation of the $\mathrm{X}$-probe hot-wires in a flow field is shown in Figure $3-1$. With mean velocity $\bar{U}_{1}$ in the $x_{1}$ direction and a flow velocity $\vec{U}(t)$, the effective cooling velocity, $U_{\text {eff }}(t)$, for each sensor can be defined.

If a sine-law cooling relation is assumed $U_{\text {eff }}$ is

$$
U_{\text {eff }}=|\vec{U}| \sin \phi
$$

with the implication that all velocities are a function of time unless denoted by an overbar which implies a long time average. Sine-law cooling inherently assumes that the component of $\vec{U}$ parallel to the $x_{3}$ direction is negligible in terms of cooling the wire. Champagne 125 has shown that the correction for deviation from sine-law cooling due to the velocity component in the $\mathrm{x}_{3}$ direction is negligible for hot-wires with a length-to-diameter ratio greater than 300. The length-to-diameter ratio for the hot-wire probe, used in this study is 333. However, the best indication of the validity of sine-law cooling is obtained from an angular calibration of the probe being used. As was shown in Figure 2-21 the sine-1aw cooling is quite adequate for the $\mathrm{X}$-probe which was used.

From Figure 3-1 it can be seen that

$$
\vec{U} \cdot \vec{W}=|\vec{U}| \cdot|\vec{W}| \dot{c o s} \phi=|\vec{U}| \cos \phi .
$$

With the use of the trig identity $\sin ^{2} \phi=1-\operatorname{cus}^{2} \psi$, Equation (3 2) becomes

$$
\sin ^{2} \phi=1-\left(\frac{\vec{U} \cdot \vec{W}}{|\vec{U}|}\right)^{2}
$$


$\vec{w}=\sin \alpha \hat{i}+\cos \alpha \hat{j}:|\vec{w}|=1$

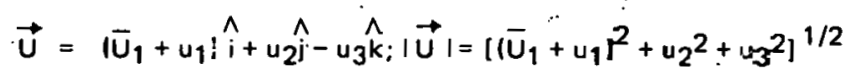

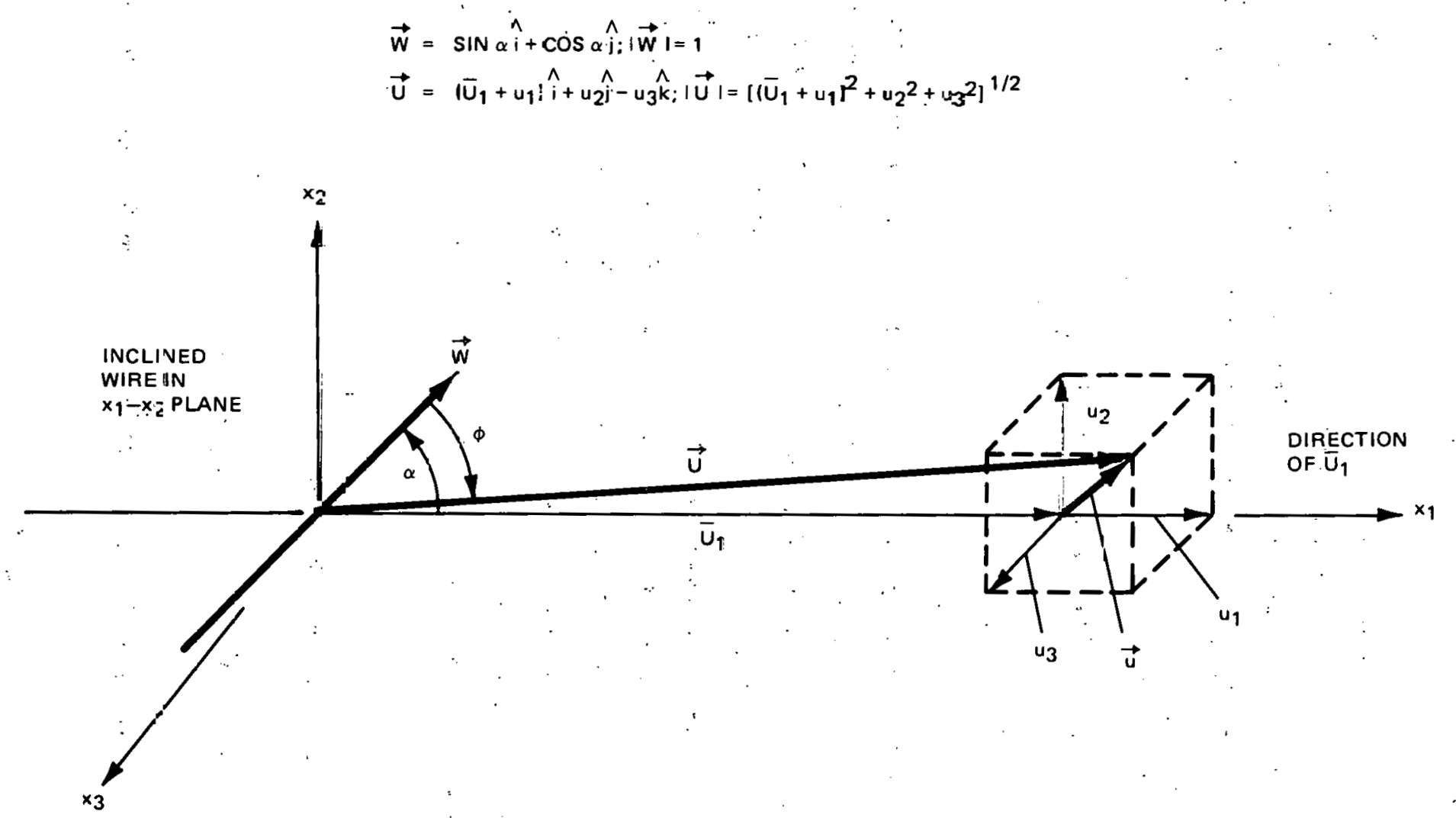

Figure 3-1. Vector Representation of Hot-Wire and Instantaneous Velocity Vectors 
Substitution of Equation (3-3) into the relationship for the effective cooling velocity along with the identities of Figure $3-1$ yields:

$$
U_{\text {eff }}^{2}=|\vec{U}|^{2}-\left[\left(\bar{U}_{1}+u_{1}\right) \cdot \cos \alpha+u_{2} \sin \alpha\right]^{2} .
$$

Substituting for $|\overrightarrow{\mathrm{U}}|$ and rearranging gives the following exact form for the effective cooling velocity based on sine-law cooling:

$$
\mathrm{U}_{\mathrm{eff}}=\overline{\mathrm{U}}_{1} \sin \alpha\left(1+\frac{\mathrm{u}_{1}}{\overline{\mathrm{U}}_{1}}-\frac{1}{\tan \alpha} \frac{\mathrm{u}_{2}}{\overline{\mathrm{U}}_{1}}\right)\left[1+\frac{\mathrm{u}_{3}^{2} /\left(\overline{\mathrm{U}}_{1}^{2} \sin ^{2} \alpha\right)}{\left(1+\frac{\mathrm{u}_{1}}{\overline{\mathrm{U}}_{1}}-\frac{1}{\tan \alpha} \frac{\mathrm{u}_{2}}{\overline{\mathrm{U}}_{1}}\right)^{2}}\right]^{1 / 2} .
$$

This exact form for the effective cooling velocity can be simplified for the conditions of low turbulence intensities and normal X-probe orientation of alpha equal to 45 degrees. Binomlal expansion of Equation (3-5) gives

$$
U_{\text {eff }}=\bar{U}_{1} \sin \alpha(1+y)\left[1+\frac{1}{2} x-\frac{1}{8} x^{2}+\frac{1}{16} x^{3}-\ldots\right] \text {, }
$$

where

$$
\mathrm{x}=\frac{\mathrm{u}_{3}^{2} /\left(\overline{\mathrm{U}}_{1} \sin \alpha\right)^{2}}{1+\mathrm{y}^{2}}
$$

and

$$
\mathrm{y}=\frac{\mathrm{u}_{1}}{\overline{\mathrm{U}}_{1}}-\frac{1}{\tan \alpha} \frac{\dot{\mathrm{u}}_{2}}{\overline{\mathrm{U}}_{1}}
$$

Under the assumption of alpha equal to 45 degrees and a turbulence intensity of less than $15 \%$, an error of less than $1 \%$ is introduced by keeping only the first 
two terms in the expansion of Equation (3-6). This approximation gives the following result for the effective cooling velocity:

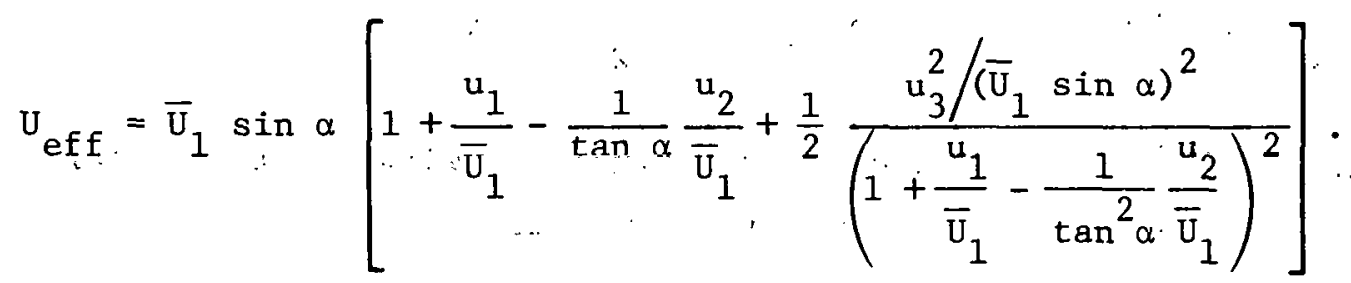

The fourth term of Equation (3-9) is approximately equal to $u_{3}^{2} / \bar{U}_{1}^{2}$ since the denominator fluctuates about ' 1 for low turbulence intensities, and $1 /\left(2 \sin ^{2} \alpha\right)$ is approximately 1 for alpha. equal to 45 degrees. Thus for low level turbulence intensities (i.e., $u_{i}{ }^{2} / U_{1}{ }^{2} \ll u_{i} / U_{1}$ ) we may neglect the third term of Equaftoñ (3-9), yielting:

$$
\tilde{U}_{\text {eff }}=\dot{\bar{U}}_{1} \sin \alpha\left(1+\frac{\mathrm{u}_{1}}{\overline{\mathrm{U}}_{1}}-\frac{1}{\tan \alpha} \frac{\mathrm{u}_{2}}{\overline{\mathrm{U}}_{1}}\right)
$$

The hot-wire response based on Equation (3-10) is then given as

$$
E=E_{0}+S^{\prime} \bar{U}_{1} \sin \alpha\left(1+\frac{u_{1}}{\ddot{U}_{1}}-\frac{1}{\tan \alpha} \frac{u_{2}}{\bar{U}_{1}}\right) \text {, }
$$

where $E$ is the hot-wire response in volts, $E_{0}$ is the iero velocity intercept of the best straight line fit through the calibration data points in the velucity range of interest and $S^{\prime}$ the slope of the best fit straight line through the calibration data points in the velocity range of interest with the wire normal to the mean flow direction, (i.e. sensitivity (volts/ft/sec)). By defining a new sensitivity constant as $S=S^{\prime} \sin (\alpha)$ the $X$-probe can be calibrated in its operating orientation with respect to the flow field. A calibration of the d.c. output of the hot-wire response in the core region of a subsonic jet makes it possible to obtain $S$, and a yaw test makes it possible to accurately determine alpha. The response in the core of the jet is just $E=E_{0}+S_{1}$ as $u_{1}=u_{2}=0$. 
The mean velocity can then be found in the flow field from Equation (3-11). by. time averaging the hot-wire response. This yields a measured mean velocity of

$$
\overline{\mathrm{U}}_{\text {meas }}=\frac{\overline{\mathrm{E}-\overline{\mathrm{E}}_{\mathrm{O}}}}{\mathrm{S}} \text {. }
$$

The error introduced into $\overline{\mathrm{U}}_{\text {meas }}$ by the fluctuating components can be estimated by substituting the actual mean velocity, $\overline{\mathrm{U}}_{\text {act }}$, into Equation (3-11) and then substituting Equation (3-11) into Equation (3-12). This yields the following " result:

$$
\overline{\mathrm{U}}_{\text {meas }}=\overline{\mathrm{U}}_{\text {act }}\left\{1+\frac{1}{2}\left[\frac{\left.\frac{\left.\mathrm{u}_{2}^{2} / \overline{\mathrm{U}}_{\text {act }} \sin \alpha\right)^{2}}{\left(1+\frac{\mathrm{u}_{1}}{\overline{\mathrm{U}}_{\text {act }}}-\frac{1}{\tan \alpha} \frac{\mathrm{u}_{3}}{\overline{\mathrm{U}}_{\text {act }}}\right)^{2}}\right]}{\ldots}\right] .\right.
$$

Since the bracketed term is always equal to or greater than 1 , the actual mean veloc1ty will be equal to or less than the measured mean velocity. Expansion of Equation (3-13) with alpha equal to 45 degrees gives

$$
\frac{\overline{\mathrm{U}}_{\text {meas }}}{\overline{\mathrm{U}}_{\text {act }}}=1+\frac{\overline{\mathrm{u}_{2}^{2}}}{\overline{\mathrm{U}}_{\text {act }}^{2}}-\frac{\overline{\mathrm{u}_{2}^{2} \mathrm{u}_{1}}}{\overline{\mathrm{U}}_{\text {act }}^{3}}+\frac{1}{2} \frac{\overline{\mathrm{u}_{3} \mathrm{u}^{2}}}{\overline{\mathrm{U}}_{\text {act }}^{3}}+\left[\begin{array}{c}
\text { terms of } \\
\text { order }\left(\frac{\mathrm{u}_{\mathrm{i}}}{\overline{\mathrm{U}}}\right) \\
\text { and higher }
\end{array}\right] \text {. }
$$

For a turbulence intensity of $15 \%$ the error in the measured mean velocity is approximately $2 \%$.

The fluctuating velocity component can be found by taking the a.c. component of Equation (3-11) for each hot-wire of the X-probe. This gives two coupled equations for the fluctuating velocity components which are

$$
\begin{aligned}
& e_{1}=S u_{1}-\operatorname{ctn} \alpha S u_{2}, \\
& e_{2}=S u_{1}+\operatorname{ctn} \alpha S u_{2} .
\end{aligned}
$$


Solving Equations (3-15) and (3-16) simultaneously for $u_{1}$ and $u_{2}$ yields:

$$
\begin{aligned}
& u_{1}=\left(e_{2}+e_{1}\right) / 2 s \\
& u_{2}=\left(e_{2}-e_{1}\right) / 2 \operatorname{sctn} \alpha .
\end{aligned}
$$

Equations (3-17) and (3-18) can be used to obtain instantaneous velocity components $\mathrm{u}_{1}$ and $\mathrm{u}_{2}$ in the $\mathrm{x}_{1}$ and $\mathrm{x}_{2}$ directions, respectively. The RMS level, autocorrelation and power spectral density of each velocity component can then be obtained from these signals.

\subsection{TECHNIQUES FOR PRESSURE MEASUREMENTS AND PRESSURE-PRESSURE CORRELATION MEASUREMENTS}

As noted in Section 1, the acoustic noise generation and propagation is very difficult to control in confined flow systems. The fluctuating pressure measured at any point in the flow field, including at the walls, will consist of both an acoustic pressure fluctuation which is transported at the speed of sound relative to the flitid velocity and a local turbulent fluid pressure fluctuation which is transported at a fluid convection velocity which is close to the mean fluid velocity. In the case where the acoustic pressure wave is significant in magnitude with respect to the turbulent fluid fluctuation, it is virtually impossible to directly obtain correct autocorrelations, power spectral densities and RMS pressure levels due to the local pressure fluctuations in the fluid turbulence. Since the acoustic pressure waves (i.e., noise) arise from flow field turbulence far upstream (i.e., flow past obstructions, through restrictions and through expansions), the frequency bandwidth lies within the trequency range of the local hydrostatic pressure fluctuations which are to be measured. This makes it impossible to separate the hydrostatic and acoustic pressure signals from a single sensor. This necessitates the use of more than one sensor to separate the superimposed acoustic and hydrostatic pressure information. The necessary signal manipulation is outlined in the following development. 
The instantaneous pressure, $F(\vec{x}, t)$, measured at any position $\vec{x}$ and time $t$ is

$$
F(\vec{x}, t)=P(\vec{x}, t)+A(\vec{x}, t)+E(t)
$$

where $P(\vec{x}, t)$ is the signal due to the local fluid pressure fluctuations at $\vec{x}$ and time $t ; A(\vec{x}, t)$ is the signal due to the acoustic pressure fluctuations at $\vec{x}$; and time $t$ and $E(t)$ is the signal due to the electronic noise of the instrumentation at time $t$. If, as we have assumed, the acoustic noise is generated far upstream or downstream (i.e., several fluid integral scale lengths) from the point of measurement, then $A(\vec{x}, t)$ will be equal in both magnitude and phase for all radial and azimuthal locations for any. given axial location [i.e.; $A(\vec{x}, t)$ becomes a function of only the axial variable $z, A(\vec{x}, t)=A(z, t)]$. This assumption makes it possible to remove the acoustic pressure contribution from the total pressure signal by subtracting two pressure signals. If the two signals are hydrodynamically uncorrelated the resultant signal will contain the complete information of the hydrostatic pressure from one point superimposed on that of the other point. The problem then is to process the signal such that the necessary information (i.e., autocorrelation, power density and RMS level) is obtained at each measured location. This becomes possible by using the coherence technique which is developed below. This approach is an extension of the subtraction method used by Wambsganss and Zaleski. (101) It has the advantage over their approach in as much as it is not limited to homogeneous flow conditions.

The autocorrelation $R_{P_{i}} P_{j}$ is defined as .

$$
\frac{\mathrm{L}^{\top} \operatorname{jm}_{\infty} \frac{1}{T} \int_{0}^{T} r_{i}(t) P_{j}(t+\bar{\tau}) d t}{\frac{1}{T}\left[\int_{0}^{T} P_{i}^{2}(t) d t\right]^{1 / 2}\left[\int_{0}^{T} P_{j}^{2}(t) d t\right]^{1 / 2}}
$$

with $i=j$. The numerator of Equation (3-20) is defined as the covariance $\left[\operatorname{Cov}_{\mathrm{p}_{i} \mathrm{p}_{j}}(\tau)\right]$ and the subscripts, $i$ and $j$, refer to the position in the flow 
channel at a specified axial location. The subtraction of two hydrodynamically uncorrelated pressure signals will yield the following covariance:

$\operatorname{Cov}_{F_{2}-F_{1}}(\tau)=T^{1+\frac{i m}{\rightarrow} \infty} \frac{1}{T} \int_{0}^{T}\left[F_{2}(t)-F_{1}(t)\right]\left[F_{2}(t+\tau)-F_{1}(t+\tau)\right] d t$.

Substitution from Equation (3-19) yields:

$$
\begin{aligned}
\operatorname{Cov}_{F_{2}-F_{1}}(\tau)= & T^{l i m} \rightarrow \frac{1}{T} \int_{0}^{T}\left\{\left[P_{2}(t)+A_{2}(t)+E_{2}(t)-P_{1}(t)-A_{1}(t)-E_{1}(t)\right]\right\} \\
& {\left[P_{2}(\tau+\tau)+A_{2}(\tau+\tau)+E_{2}(\tau+\tau)-P_{1}(\tau+\tau)-A_{1}(t+\tau)\right.} \\
& \left.-E_{1}(t+\tau)\right] d t .
\end{aligned}
$$

Applying the following assumptions to Equation (3-22):

1. The acoustic pressure is equal in magnitude and,phase at all points in any axial plane, i.e., $A_{1}(t)=A_{2}(t)$ and $A_{1}(t+\tau)=A_{2}(t+\tau)$;

2. There is no correlation between the hydrostatic pressure, $P_{i}$, and the electronic instrument noise, $E_{i}$, i.e.,

$$
\int_{0}^{T} P_{i}(t) E_{j}(t) d t=0 \text { for all } i \text { and } j
$$

3. There is no correlation between the hydrostatic pressures at positions 1 and 2 , 1.e.,

$$
\int_{0}^{T} P_{i}(t) P_{j}(t+r) d t=0 \text { for } i \neq j
$$


4. There is no correlation between any two electronic noise signals, i.e.,

$$
\begin{aligned}
& \int_{0}^{T} E_{i}(t) E_{j}(t+\tau)=0 \text { for } i \neq j, \\
& \text { reduces it to the form: } \\
& \operatorname{Cov}_{F_{2}-F_{1}(\tau)=} T^{1 i_{\rightarrow}} \frac{1}{T} \int_{0}^{T}\left[P_{2}(t) P_{2}(t+\tau)+E_{2}(t) E_{2}(t+\tau)\right. \\
& \left.\quad+P_{1}(t) P_{1}(t+\tau)+E_{1}(t) E_{1}(t+\tau)\right] d t .
\end{aligned}
$$

The right side of Equation (3-23) can be separated into four separate integrals of which each is a simplified covariance:

$$
\operatorname{Cov}_{F_{2}-F_{1}}(\tau)=\operatorname{Cov}_{P_{2} P_{2}}(\tau)+\operatorname{Cov}_{P_{1} P_{1}}(\tau)+\operatorname{Cov}_{E_{2} E_{2}}(\tau)+\operatorname{Cov}_{E_{1} E_{1}}(\tau) \ldots
$$

By using a third pressure signal which is hydrodynamically uncorrelated with either of the other two signals and taking the three possible signal subtractions, two more equations similar to Equation (3-24) are-obtained:

$$
\begin{aligned}
& \operatorname{Cov}_{F_{3}-F_{1}}(\tau)=\operatorname{Cov}_{P_{3} P_{3}}(\tau)+\operatorname{Cov}_{P_{1} P_{1}}(\tau)+\operatorname{Cov}_{E_{3} E_{3}}(\tau)+\operatorname{Cov}_{E_{1} E_{1}}(\tau), \\
& \operatorname{Cov}_{F_{3}-F_{2}}(\tau)-\operatorname{Cov}_{P_{3} P_{3}}(\tau)+\operatorname{Cov}_{P_{2} P_{2}}(\tau) 1 \operatorname{Cov}_{E_{3} E_{3}}(\tau)+\operatorname{Cov}_{E_{2} E_{2}}(\tau) .
\end{aligned}
$$

Equations (3-24), (3-25) and (3-26) result in three equations with three unknown covariances, $\operatorname{Cov}_{\mathrm{P}_{3} \mathrm{P}_{3}}(\tau), \operatorname{Cov}_{\mathrm{P}_{2} \mathrm{P}_{2}}(\tau)$ and $\operatorname{Cov}_{\mathrm{P}_{1} \mathrm{P}_{1}}(\tau)$. Solving for these three $\operatorname{Cov}_{P_{1} P_{1}}(\tau)=\frac{1}{2}\left[\operatorname{Cov}_{F_{2}-F_{1}}(\tau)-\operatorname{Cov}_{F_{3}-F_{2}}(\tau)+\operatorname{Cov}_{F_{3}-F_{1}}(\tau)-2 \operatorname{Cov}_{E_{1} E_{1}}(\tau)\right]$, 
$\operatorname{Cov}_{P_{2} P_{2}}(\tau)=\frac{1}{2}\left[\operatorname{Cov}_{F_{2}-F_{1}}(\tau)-\operatorname{Cov}_{F_{3} F_{1}}(\tau)+\operatorname{Cov}_{F_{3}-F_{1}}(\tau)-2 \operatorname{Cov}_{E_{2} E_{2}}(\tau)\right]$,

$\operatorname{Cov}_{P_{3} P_{3}}(\tau)=\frac{1}{2}\left[\operatorname{Cov}_{F_{3}-F_{1}}(\tau)-\operatorname{Cov}_{F_{2}-F_{1}}(\tau)+\operatorname{Cov}_{F_{3}-F_{2}}(\tau)-2 \operatorname{Cov}_{E_{3} E_{3}}(\tau)\right]$.

Equations (3-27), (3-28) and (3-29) can be used to calculate the autocorrelation of the fluctuating turbulent fluid pressure from the three independent sensors, $F_{1}(t), F_{2}(t)$ and $F_{3}(t)$ for inhomogeneous turbulent pressure fields contaminated with acoustic noise. The power spectral density is then obtained by Fourier transforming the autocorrelation:

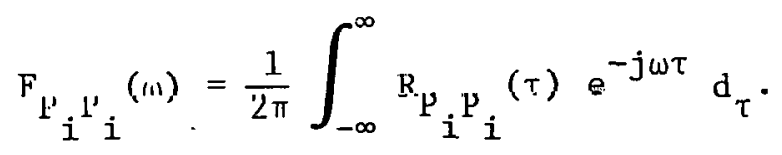

The RMMS level of the hydrostatic pressure fluctuations can be obtained by taking the square-root of the covariance at tau equal to zero:

$$
\left(\overline{\left(\mathrm{P}_{i}^{2}\right.}\right)^{1 / 2}=\left[\operatorname{Cov}_{\mathrm{P}_{i} \mathrm{P}_{i}}(\tau=0)\right]^{1 / 2}
$$

'I'he cross-correlation of two pressure signals is given by Equation (3-20) with $i$ not equal to $j$ and can be found by a method similar to that for the autocorrelation. The cross-correlation of the hydrostatic pressure at points 1 and 2 can be found by subtracting a third hydrodynamically uncorrelated pressure signal $F_{3}(t)$ from both $F_{2}(t)$ and $F_{1}(t)$. The covariance of these two difference signals gives

$\operatorname{Cov}_{F_{1-3} F_{2-3}}(\tau)=T^{\lim } \rightarrow \frac{1}{T} \int_{0}^{T}\left[F_{1}(t)-F_{3}(t)\right]\left[F_{2}(t+\tau)-F_{3}(t+\tau)\right] d t$.

Subst1tuting $F_{i}(t)$ from Equation (3-19) into Equation (3-32) and applying assumptions $1 ., 2 ., 4 .$, and 
5. there is no correlation between the hydrostatic pressures at positions 1 and 3 , and at positions 2 and 3 , i.e.,

$$
\int_{0}^{T} P_{1}(t) P_{3}(t+\tau) d t=\int_{0}^{T} P_{2}(t) P_{3}(t+\tau) d t=0
$$

yields:

$$
\operatorname{Cov}_{1-3} F_{2-3}(\tau)-\operatorname{Cov}_{P_{1} P_{2}}(\tau)+\operatorname{Cov}_{P_{3} P_{3}}(\tau)+\operatorname{Cov}_{E_{3} E_{3}}(\tau)
$$

Rearranging Equation (3-33) gives the covariance of the hydrostatic pressure between points 1 and 2 in terms of the known covariances, i.e. the covariance of the two difference signals, the covariance of the electronic noise and the covariance of the hydrostatic pressure as found from Equation. (3-29):

$$
\operatorname{Cov}_{P_{1} P_{2}}(\tau)=\operatorname{Cov}_{F_{1-3} F_{2-3}}(\tau)-\operatorname{Cov}_{E_{3} E_{3}}(\tau)-\operatorname{Cov}_{P_{3} P_{3}}(\tau)
$$

The cross-correlation of the in-stream fluctuating static pressure with the fluctuating static pressure at the wall can be obtained by this general method as well as the cross-correlation of the in-stream fluctuating static pressure at two separate locations.

\subsection{TECHNIQUES FOR PRESSURE-VELOCITY CORRELATION MEASUREMENTS .}

In order to get a better understanding of the effect of the flow field on the wall pressure it is of interest to measure the fluctuating velocity components and their correlation to the wall pressure. An approach similar to that of subsection 3.2 was used to obtain these correlations. The cross correlation of the wall pressure and velocity is given as

$$
\mathrm{R}_{\mathrm{Pu}}(\tau)=\frac{\mathrm{T}_{i}^{\lim } \rightarrow \frac{1}{\mathrm{~T}} \int_{0}^{\mathrm{T}} \mathrm{P}(\mathrm{t}) \mathrm{u}_{i}(t+\tau) \mathrm{dt}}{\mathrm{T}^{\lim _{\rightarrow}}\left[\frac{1}{\mathrm{~T}} \int_{0}^{\mathrm{T}} \mathrm{P}^{2}(\mathrm{t}) \mathrm{dt}\right]^{1 / 2}\left[\frac{1}{\mathrm{~T}} \int_{0}^{\mathrm{T}} \mathrm{u}_{i}^{2}(t) \mathrm{dt}\right]^{1 / 2}},
$$


where $u_{i}(t)$ is the velocity component in the ith-direction which is being correlated with the wall pressure $P(t)$. The covariance of the pressure signal as given by Equation (3-19) and the velocity signal yields:

$$
\operatorname{Cov}_{F u_{i}}(\tau)=T^{\lim } \rightarrow \infty \frac{1}{T} \int_{0}^{T}[P(t)+A(t)+E(t)]\left[u_{i}(t+\tau)+u_{E}(t+\tau)\right] d t, \quad(3-36)
$$

where $u_{E}(t+\tau)$ is the electronic noise of the velocity signal. Application of the following assumptions:

1. There is no correlation between any two electronic noise signals, i.e.,

$$
\int_{0}^{T} E(t) u_{E}(t+\tau) d \cdot t=0
$$

2. The electronic noise is uncorrelated with either the velocity field or pressure, i.e.,

$$
\int_{0}^{T} P(t) u_{E}(t+\tau) d t=\int_{0}^{T} A(t) u_{E}(t+\tau) d t=\int_{0}^{T} E(t) u_{i}(t+\tau) d t=0
$$

3. The acoustic field originates far from the point of measurement and is uncorrelated with the local velocity components, i.e.,

$$
\int_{0}^{T} A(t) u_{1}(t+T) \dot{d} t=0
$$

yiclds

$$
\operatorname{Cov}_{\mathrm{Fu}}(\tau)=\mathrm{T}^{\lim }+\frac{1}{\mathrm{~T}} \int_{0}^{\mathrm{T}} \mathrm{F}(L) \mathrm{u}_{i}(L+\tau) d \tau
$$


The right side of Equation (3-37) is just the covariance of the hydrostatic pressure and velocity component of interest. Thus, the covariance of the pressure signal $F(t)$ and the velocity signal $u_{i}(t)$ will give the desired covariance of the hydrostatic pressure and the velocity. 


\section{SINGLE POINT MEASUREMENTS}

\subsection{SINGLE POINT MEASUREMENTS}

Preliminary velocity measurements were made with a pitot tube. The pitot tube was constructed out of a 0.060-in. diameter SS support tube with a number 25 hypodermic needle mounted in the end. The static pressure was monitored on the outer wall of the flow channel wi.th a flush mounted wall tap at the same axial position as the pitot tube tip. The dynamic pressure head was measured with a $\mathrm{U}$-tube water manometer using the static pressure tap as the second leg of the manometer.

Mean velocity profiles were measured at several axial locations and several azimuthal locations. These measurements were made to ensure that the flow was fully developed and that the spiders supporting the central rod were not affecting the mean velocity profile.

The mean velocity profiles taken at different axial positions showed no perceivable change in their shape. This information infers that the flow field is fully developed as the profile is not changing as the flow proceeds down the channel.

The mean velocity profile at different azimuthal positions also showed no perceivable change. This implies that the supports for the central rod are not affecting the mean flow at the downstream locations where velocity and pressure measurements were to be taken.

Figure 4-1 shows the mean velocity profile measured with the pitot tube. The transverse shown was taken at axial position 2 which is between the two diametrically opposed wall pressure transducers. Also included in Figure 4-1 is the mean velocity profile measured at the same position with the $\mathrm{X}$-probe 1249G-T1.5. The pitot tube and $\mathrm{X}$-probe data agree with each other to $\pm 2 \%$. Also shown in Figure 4-1 is the mean velocity profile measured by Brighton and Jones 126 under similar flow conditions. 


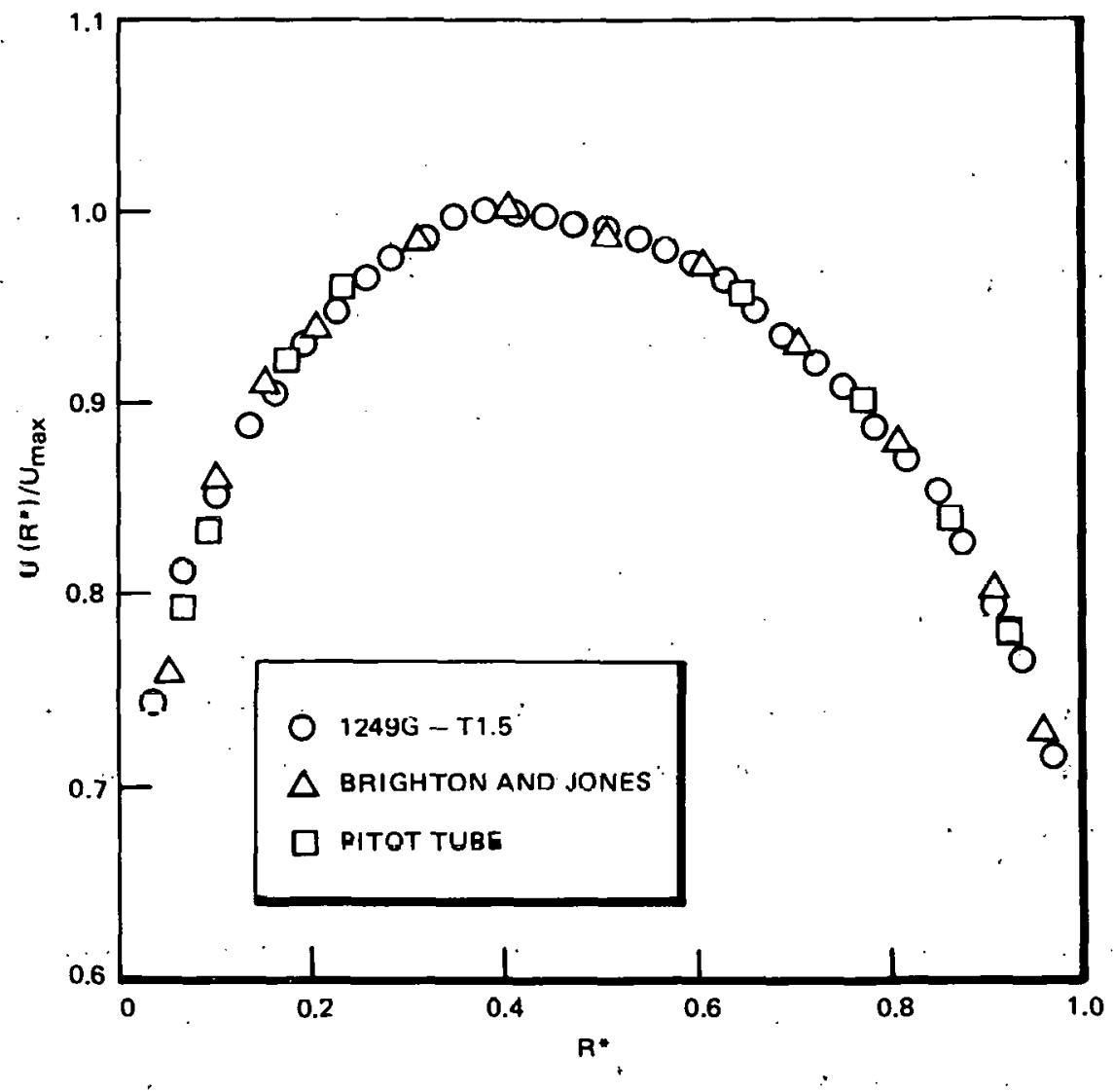

Figure 4-1. Average Velocity Profile at a Mean Velocity of $200 \mathrm{ft} / \mathrm{sec}$

The mean velocity profile is relatively flat with a mean to peak velocity ratio of

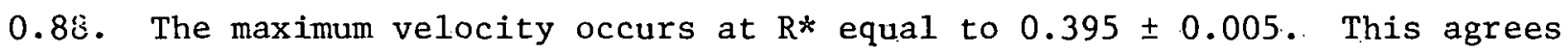
quite well with the data given hy Rrighton and Jones ${ }^{126}$ of. $U_{\max }$ at Rh eyual LU 0.38 for $R_{1} / R_{2}=0.232$. Kays and Leung ${ }^{127}$ give the Jocation $n$ if the maximum velocity in an annular flow field as $\left(R_{m}-R_{1}\right) /\left(R_{2}-R_{m_{m}}\right)=\left(R_{1} / R_{2}\right)^{0.343}$ : This corresponds to $\mathrm{R}^{*}$ equal to 0.377 .

Figure 4-2 shows the mean velocity profile nondimensionalized by the friction velocity, $u_{\tau}$, versus the nondimensional variable $y^{*} u_{\tau} / v$ where $y$ is the distance from the wall, $v$ the kinematic viscosity of the fluid and $u_{\tau}$ the friction velocity at the inner and outer walls respectively. The friction velocity measurements and values are detailed in Subsection 4.2 . 


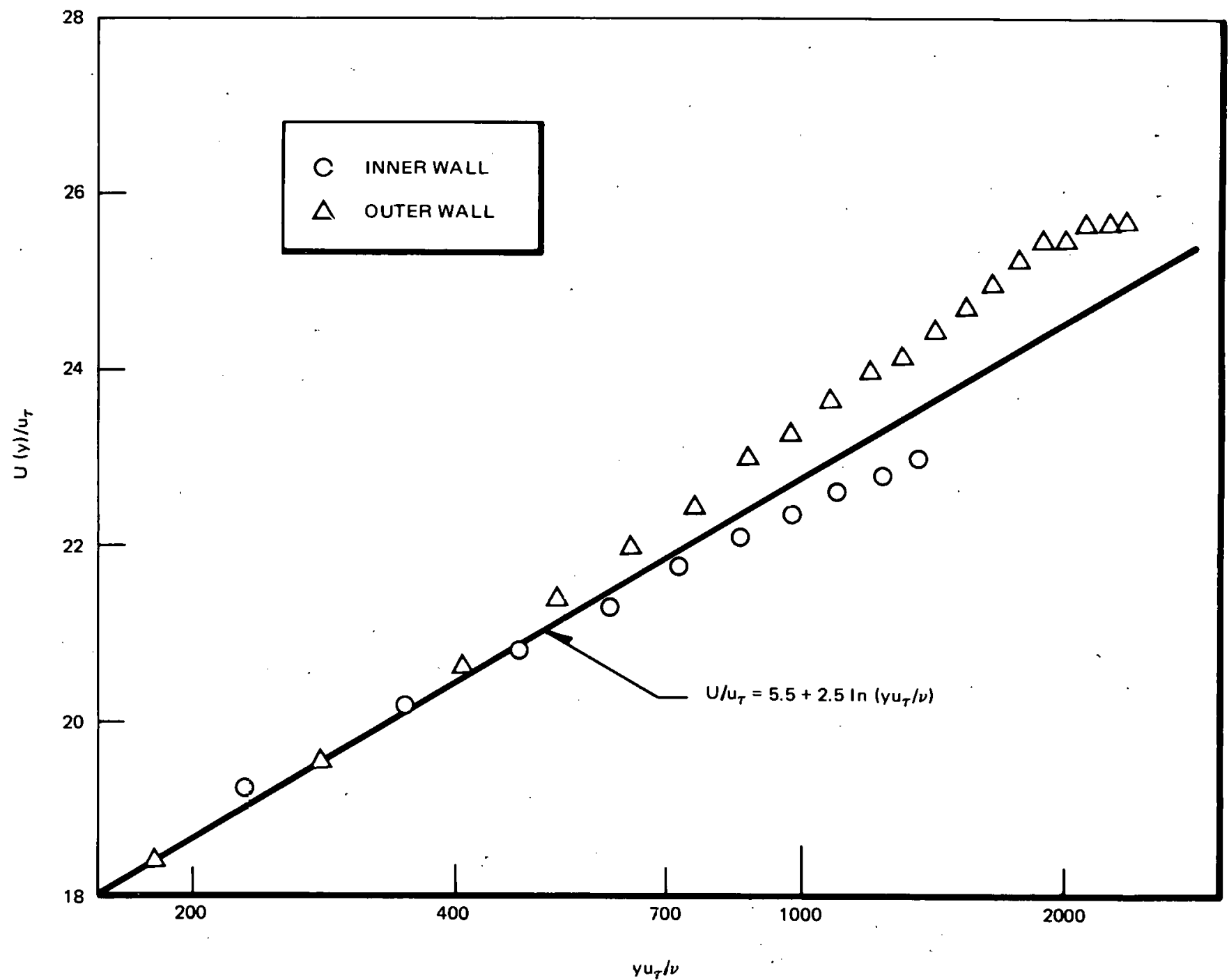

Figure 4-2. Law of the Wall Mean Velocity Profile 
The solid line on Figure 4-2 is the universal velocity distribution law for very large Reynolds numbers which is given as $U(y) / u_{\tau}=5.5+2.5 \ln \left(y * u_{\tau} / v\right)$ by Schlichting ${ }^{128}$. The data are in good agreement with this universal velocity distribution. The trends for the outer wall profile to rise a little faster than the universal velocity distribution and for the inner wall to fall a little faster than the universal velocity distribution were also noted by Brighton and Jones $^{126}$ and Lawn and El1iott. ${ }^{129}$ These changes from the universal velocity distribulion are dependent upon the amount of curvature of the wall. Brighton and Jones $^{126}$ and Lawn and Elliott ${ }^{129}$ demonstrate the change in the velocity profile near the inner wall for different radius ratios. The profile near the inner wall drops off faster as $\mathrm{R}_{1}$ becomes smaller.

The mean velocity profile and universal velocity distribution data demonstrates a fully developed flow field which agrees quite well with puhlished information on fully developed flow in annular. flow channels.

\subsection{SINGLE POINT TURBULENT VELOCITY MEASUREMENTS}

The single point turbulent velocity measurements were made with a TSI model 1249G-T1.5 X-probe. The calibration and mounting were detailed in Subsection 2.4. The acquistitions of the voltage signals which are proportional to the instantaneous velocity are outlined in Subsection 3.1. A block diagram of the velocity acquisition system is shown in Figure 4-3.

The velocity components measured with $\mathrm{X}$-probe $1249 \mathrm{G}-\mathrm{T} 1.5$ were those in the radial and axial directions which are $u_{r}(t)$ and $u_{z}(t)$, respectively.

The Reynolds stress distribution, defined as $\overline{u_{z}(t) * u_{r}(t)}$, is shown in Figurc 4-4. The solid line is the distribution calculated by Brighton and .Tnnes. 126 The data are extrapolated to the wall using the curvature of the calculated values to obtain the wall shear stresses and the friction velocities. The friction velocity is defined as $u_{\tau}\left(R^{*}\right)=\left[\overline{\left(u_{z}(t) u_{r}(t)\right.}\right]^{1 / 2}$. The friction velocity of the inner wal1, $R^{*}=0.0$, is denoted as $u_{\tau_{1}}$ and that of the outer wall, $R^{*}=1.0$, as $u_{\tau}$. The wall shear stress is given as $\tau_{i}=\left(u_{\mathrm{T}}^{2} \rho_{i}\right) / g_{c}$ where the subscript i. is 1 for the inner wall and 2 for the outer wall, $\rho$ is the density of the fluid and $\mathrm{g}_{\mathrm{c}}$ the conversion constant equal to $32.21 \mathrm{~b}_{\mathrm{m}}-\mathrm{ft} / 1 \mathrm{~b}_{\mathrm{f}}-\mathrm{sec}^{2}$. 


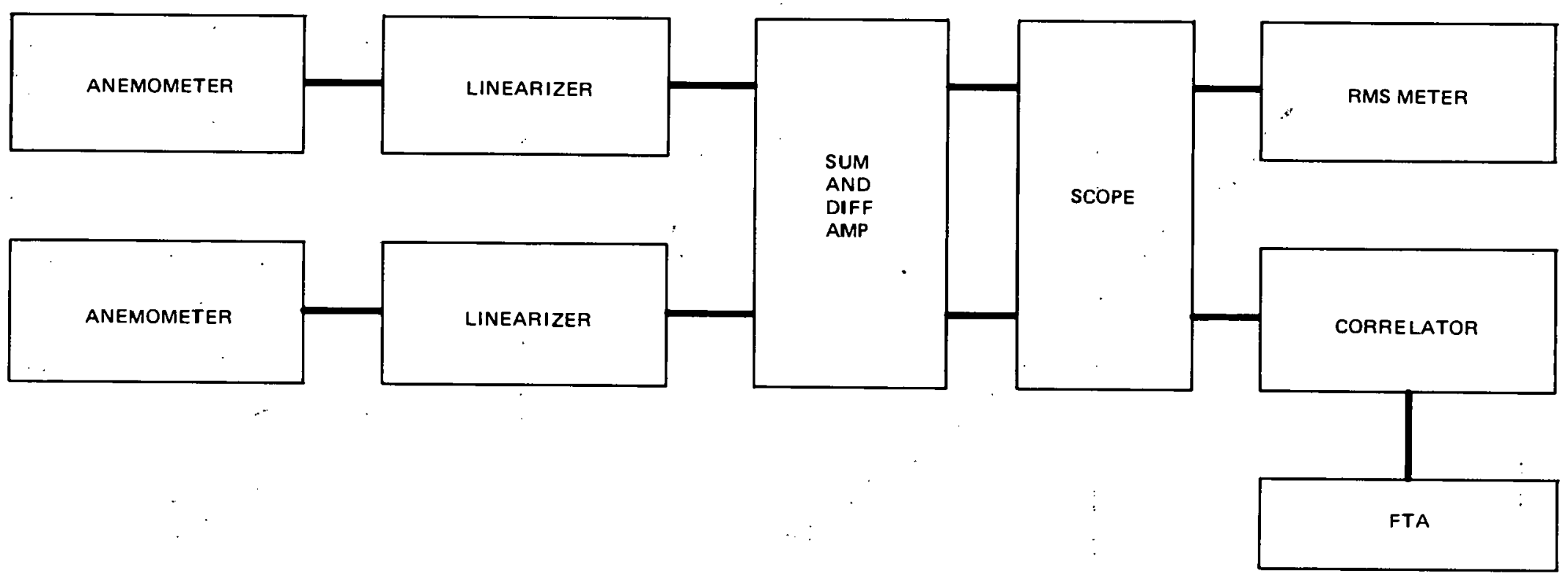

Figure 4-5. Block Diagram of the Velocity Data Acquisition System 


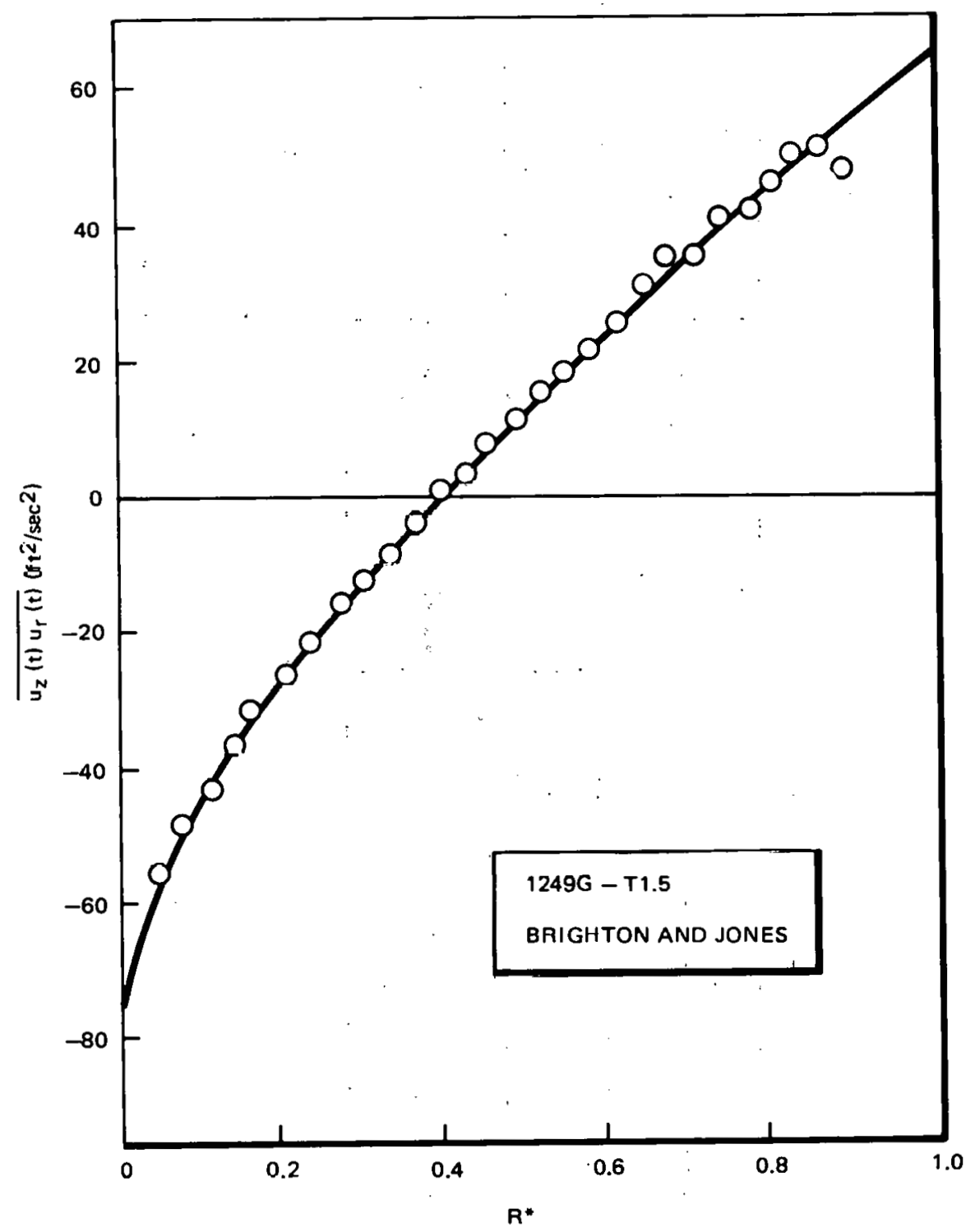

Figure 4-4. Reynolds Stress Distribution

The measured friction velocity at the inner wal1 is $8.83 \pm 0.15 \mathrm{ft} / 3 \mathrm{ec}$ and that at the outer wall is $7.94 \pm 0.15 \mathrm{ft} / \mathrm{ser}$. The reeultant wall slieai slress at the inner wall is $0.0349 \pm 0.0012$ in. $\mathrm{H}_{2} \mathrm{O}$ and that at the outer wall is 0.0282 \pm 0.0010 in. $\mathrm{H}_{2} \mathrm{O}$.

It has been shown by Lawn and Elliott ${ }^{129}$ that the zero shear stress and the point of maximum velocity do not coincide for fully developed annular flow. Their data show a consistent difference of about $2 \%$ in the location of zero 
shear stress and maximum velocity with the latter being closer to the outer wall for smooth surfaces. They also note that the location of these two maximum can be switched such that the zero shear position lies closer to the outer wall by roughening the center rod.

The location of $\overline{u_{z}(t) u_{r}(t)}=0.0$ is $R^{*}=0.385 \pm 0.005$ for this study. This is. consistent with the information given by Lawn and Elliott ${ }^{129}$ for smooth surfaces, as the point of maximum velocity, $R^{*}=0.395 \pm 0.005$ is slightly greater than the location of zero shear stress.

It appears that the rclative locations of the point of maximum velocity and the point of zero shear are highly dependent on the condition of the walls of the flow channel. Therefore, it is impossible to draw any conclusive information about the relative positions without quantifying the surface conditions. The data presented here are not intended to clarify the relative positions of the maximum velocity and zero shear as no detailed study toward this goal was undertaken. It does polnt out that the velocity flow field data are in agreement with other reported measurements on fully developed turbulent flow in annular flow channels. Therefore, the hydrostatic pressure fluctuation statistics in this same flow field should be typical of those found in any fully developed turbulent annular flow field.

The single point velocity data are scaled by the algebraic mean friction velocity and the distance between the walls. The algebraic mean friction velocity is defined as

$$
u_{\tau_{o}}=\frac{R_{1} u_{\tau_{1}}+R_{2} u_{\tau_{2}}}{R_{1}+R_{2}}
$$

An algebraic mean wall shear stress is simllarly defined as

$$
\tau_{0}=\frac{R_{1} \tau_{1}+R_{2} \tau_{2}}{R_{1}+R_{2}}
$$


The mean friction velocity, $u_{\tau_{0}}$, is $8.11 \pm 0.15 \mathrm{ft} / \mathrm{sec}$ and the mean wall shear stress, $\tau_{0}$, is $0.0295 \pm 0.0011$ in. $\mathrm{H}_{2} \mathrm{O}$.

The above mean value of $\tau_{0}$ gives $\tau_{1} / \tau_{0}=1.18 \pm 0.06$ and $\tau_{2} / \tau_{0}=0.956 \pm 0.05$. The values calculated by Schumann 71 are $\tau_{1} / \tau_{0}=1.24$ and $\tau_{2} / \tau_{0}=0.953$ which are in quite good agreement: with the measured values. Lawn and E1liott ${ }^{129}$ give $\tau_{1} / \tau_{2}=1.42$ For $R_{1} / R_{2}=0.176$ and $\tau_{1} / \tau_{2}=1.16$ for $R_{1} / R_{2}=0.396$. The measured values for $\tau_{1}$ and $\tau_{2}$ give $\tau_{1} / \tau_{2}=1.24 \pm 0.05$ which is consistent with the data of Lawn and E1liott 130 but somewhat lower than an interpolated value of 1.33 from Lawn and Elliott's data.

One of Schumann's control parameters was $\bar{U} / u_{\tau_{0}}=30$. This is considerably larger than the measured value of $24.7 \pm 0.7 .^{\circ}$ The discrepancy is not clear, bul it is thought that Schumann's value is too large as the measured values of $u_{\tau_{1}}$ and $u_{\tau_{2}}$ are in agreement with other published experimental data. 126,128 Schumann's friction velocity based on his value of $\bar{U} / u_{\tau_{0}}$ is about $20 \%$ smaller than that measured experimentally.

The RMS intensity level of the fluctuating velocity components 1 s shown in Figures 4-5 and 4- $f$. Rnth the axial component, ${ }_{z}$, dud Lhe radial component, $u_{r}$, are normalized by the algebraic mean friction velocity. $u_{\tau}$. The profiles agree wel1 wich the measurements of Brighton and Jones ${ }^{126}$, Tiawh and Elliott ${ }^{130}$ and Rehme ${ }^{1.31}$, but are well helow thnse of Schumann ${ }^{71}$. The axial cumpunent of velocity calculated by Schumann is 20 to $30 \%$ greater than that measured experimentally, while the radial component is 60 to $80 \%$ larger. The genera1 shape and the location of the minimum value agree well, but the magnitude is coneidcrably different. The data of Brighton and Jones ${ }^{126}$ are shown in Figures 4-5 and 4-6 for comparison. Brighton and Jones data are for a radius ratio of 0.562 which is larger than that used in this study. They show no Reynolds number dependency on the profile, but do show slightly lower intensity level for $u_{z}$ for increasing $R_{2} / R_{1}$ and a shift of the maximum toward $R^{*}=0.5$ for increasing $R_{2} / R_{1}$. They alsu show a slightly higher intensity level for $u_{r}$ as $R_{2} / R_{1}$ increases. All of these trends are in agreement with the data shown in Figures 4-5 and 4-6.

Figures 4-7 and 4-8. show the power spectral density of the two velocity components $u_{z}$ and $u_{r}$. The data are normalized by the wave number, $k$, and an 


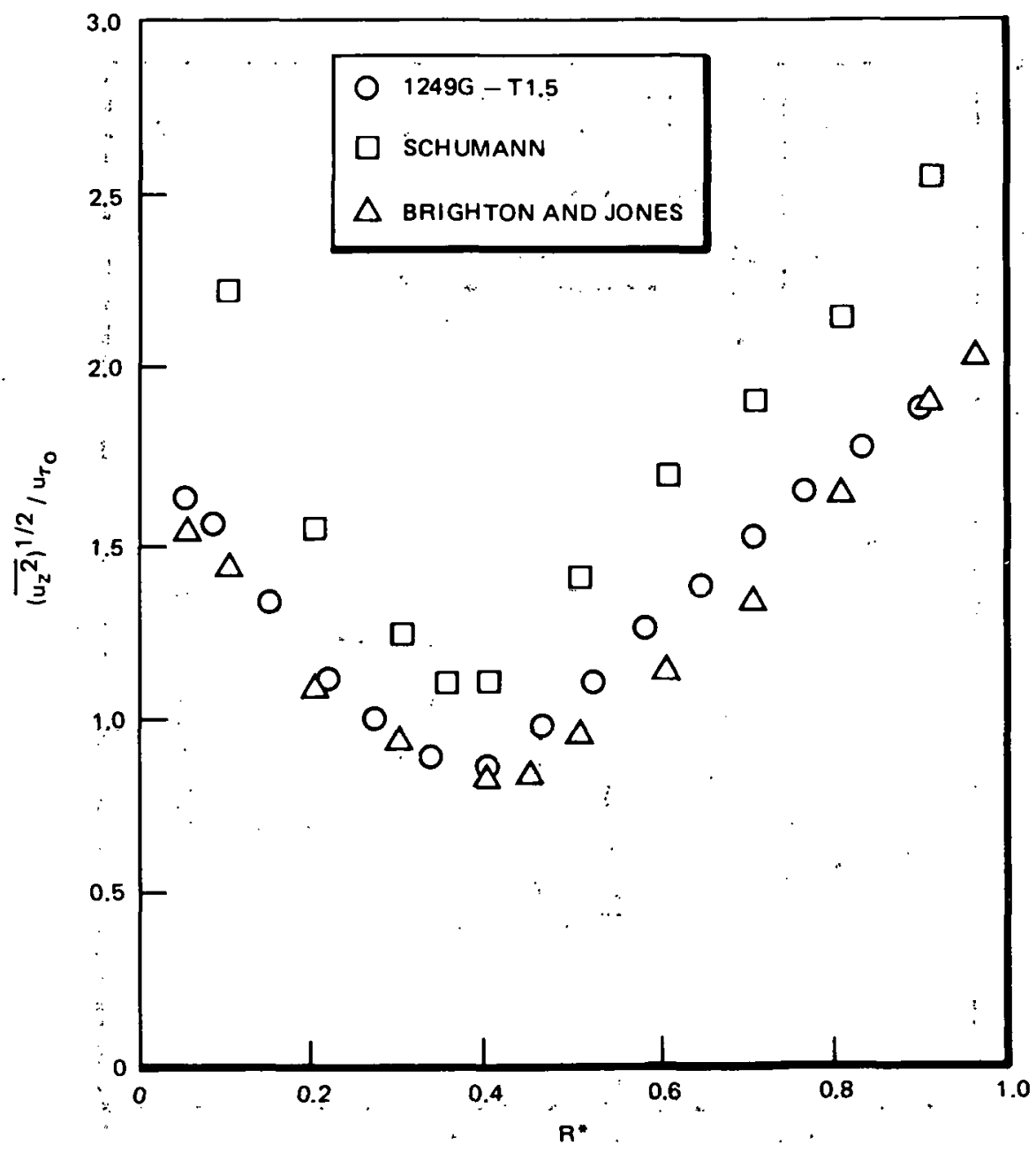

Figure:4-5. Radial Distribution of the Turbulent Axial Velocity Component

equivalent pipe radius a. The value a is defined as the distance from the wall to the point of maximum velocity, which corresponds to the radius of a pipe for fully developed pipe flow. : This value was used sc that a comparison with existing spectra from pipe flow data could be made.

The data of Figures 4-7 and 4-8 are at radial locations between the outer wall , and the maximum.velocity. Also.shown are the data of Lawn $^{132}$ for fully developed flow in a pipe. There is reasonably good agreement between the two measurements. The familiar $-5 / 3$ slope, indicative of the inertial subrange in the kolmogorov range of local similarity, is in very good agreement with the pipe flow data of 


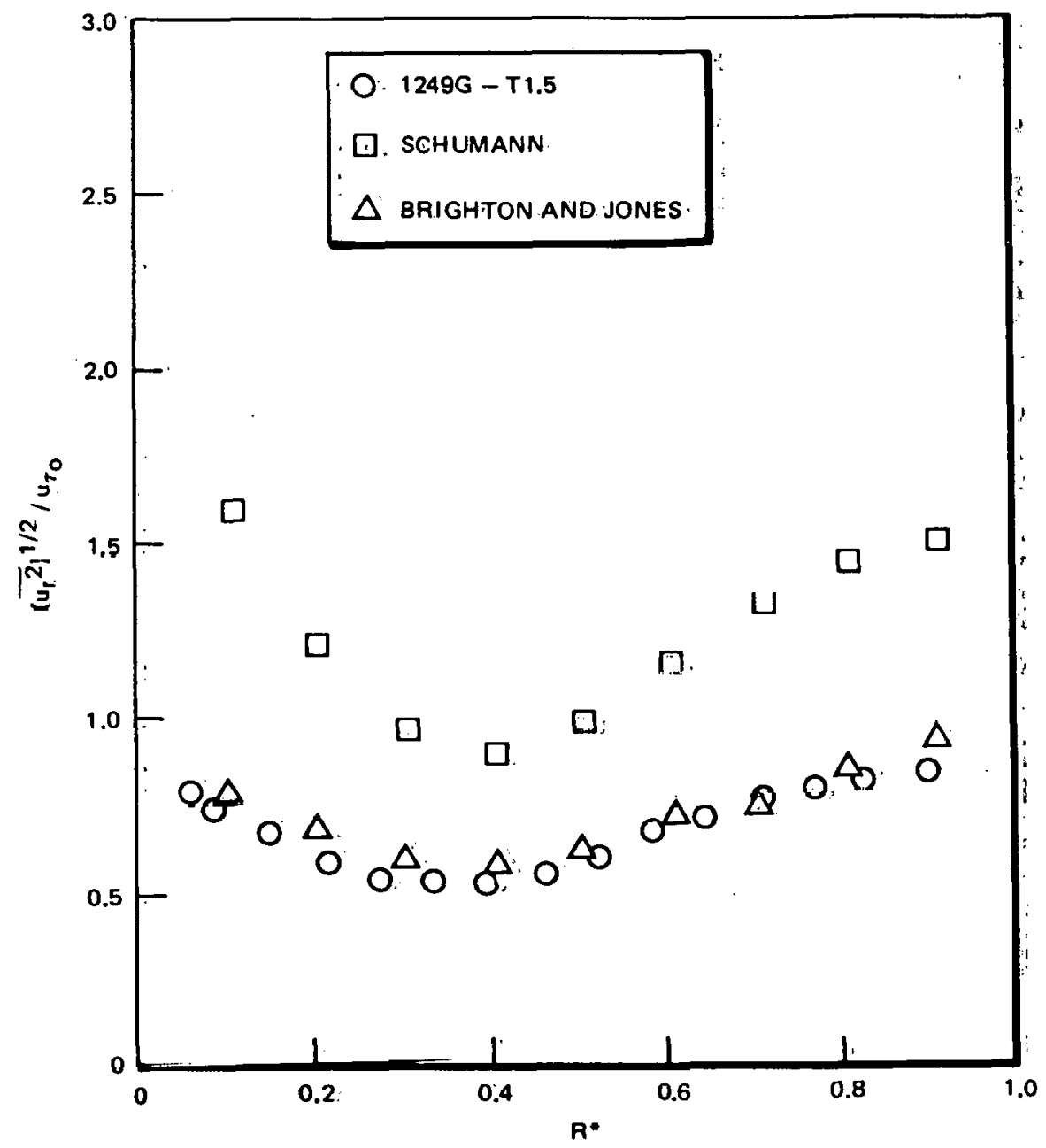

Figure 4-6. Radial Distribution of the Turbulent Radial Velocity Component

Lawn. The lower frequency shows that the annular flow gcometry cunlains more energy in this region. This may be due to the differences in curvature between the annular flow system outer wall and the wall of the pipe as defined here. The nondimensionalization used for the annular flow system will have the effect of making the equivalent point of measurement in a pipe move toward the center of the pipe. This will tend to give a larger contribution to the low frequency spectra as more large scale eddies are in existence as the point of measurement is moved toward the center. The result will be a larger contribution to the lower frequency end of the spectra. Lawn's data in general show the low frequency end increasing as the point of measurement moves toward the center of the 


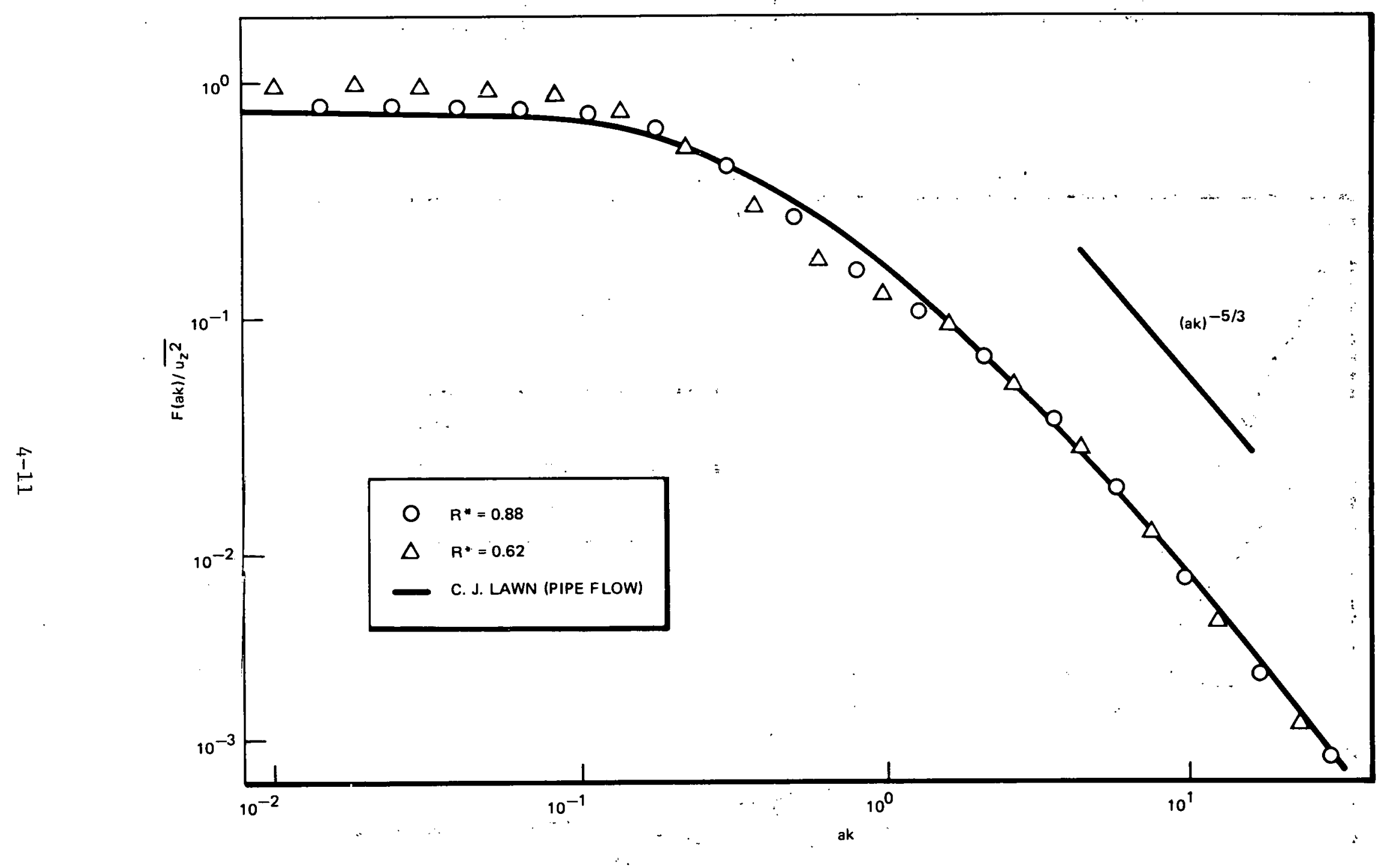

Figure 4-7. Power Spectral Density of the Axial Component of the Turbulent velocity Field at $\overline{\mathrm{U}}=200 \mathrm{ft} / \mathrm{sec}$ 


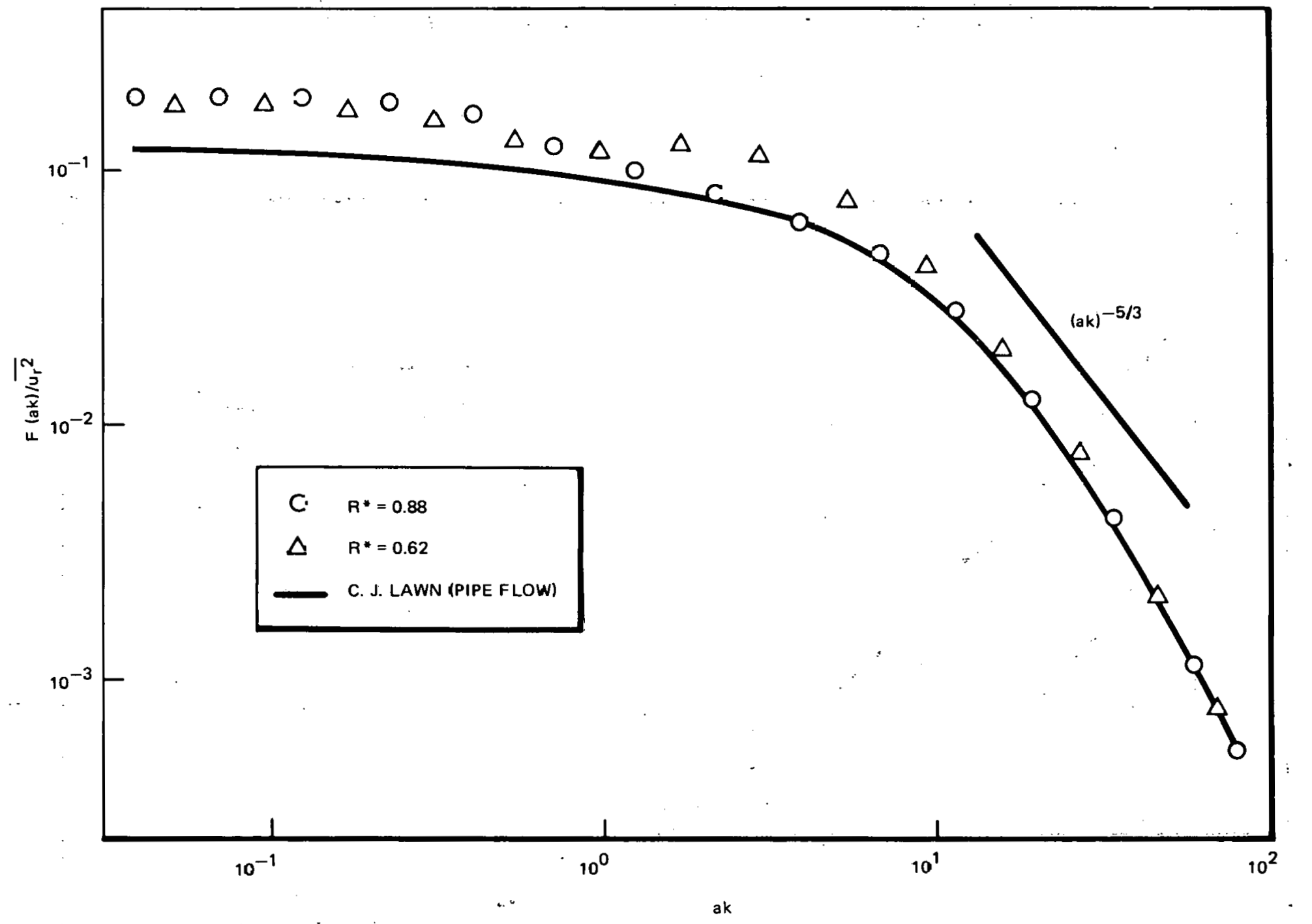

Figure 4-8. Power Spectral Density of the Radial Component of the Turbulent Ve-ccity Field at $\bar{U}=200 \mathrm{ft} / \mathrm{sec}$ 
pipe, which supports this argument for the difference in the PSD's at low frequency.

Only the spectra at "two locations are shown $\left(\mathrm{R}^{*} \stackrel{\dot{=}=}{=} 0.62\right.$ and 0.88$)$. "No extensive investigation of the velocity spectra was undertaken. The data shown in Figures 4-7 and 4-8 are representative of the measurements made. The intent of the velocity. spectra measurements were to show that there were no perturbations in the flow field turbulence due to the supporting structure of the center rod and that the velocity field was consistent with known information on spectral content.

The single point turbulent velocity meașurements show RMS magnitudes', RMS profiles and shear stress distributions which are in good agreement with existing experimental information. There is, however; a significant discrepancy with the RMS magnitudes calculated by Schumann. ${ }^{71}$ The general shape of Schumann's RMS profiles are in good agreement with the experimental measurements presented here. The results of these measurements indicate a completely developed turbulent flow field which contains turbulent velocity statistics which agree with existing information within the limits of the measurement errors.

\subsection{SINGLE POINT FLUCTUATING STATIC PRESSURE MEASUREMENTS}

The single point fluctuating static pressure measurements were made with piezoelectric pressure transducers at the wall and a bleed type pressure transducer in the flow field. The wall pressure transducer mounting and calibration is detailed in Suberction 2,2. and that of the in-stream bleed pressure transducer in Subsection 2.3. A block diagram of the wall pressure data acquisition system is shown in Figure 4-9 and that of the bleed pressure transducer in Figure 4-10. The data analysis methods used for the single point pressure measurements are outlined in Subsection 3.2.

The RMS pressure level is typically nondimensionalized by either the dynamic pressure, $q$, or the wall shear stress, $\tau$. Typical ranges as reported by Willmarth $(104,108,110)$ are $4.5-7.5 \times 10^{-3}$ for $\mathrm{P}_{\mathrm{rms}} / \mathrm{q}$ and 2.0 to 3.5 for $\mathrm{P}_{\mathrm{rms}} / \tau$. These results are for measurements on flat plates, on the outer surface of a rod in $a$ wind tunnel and pipe flow. 


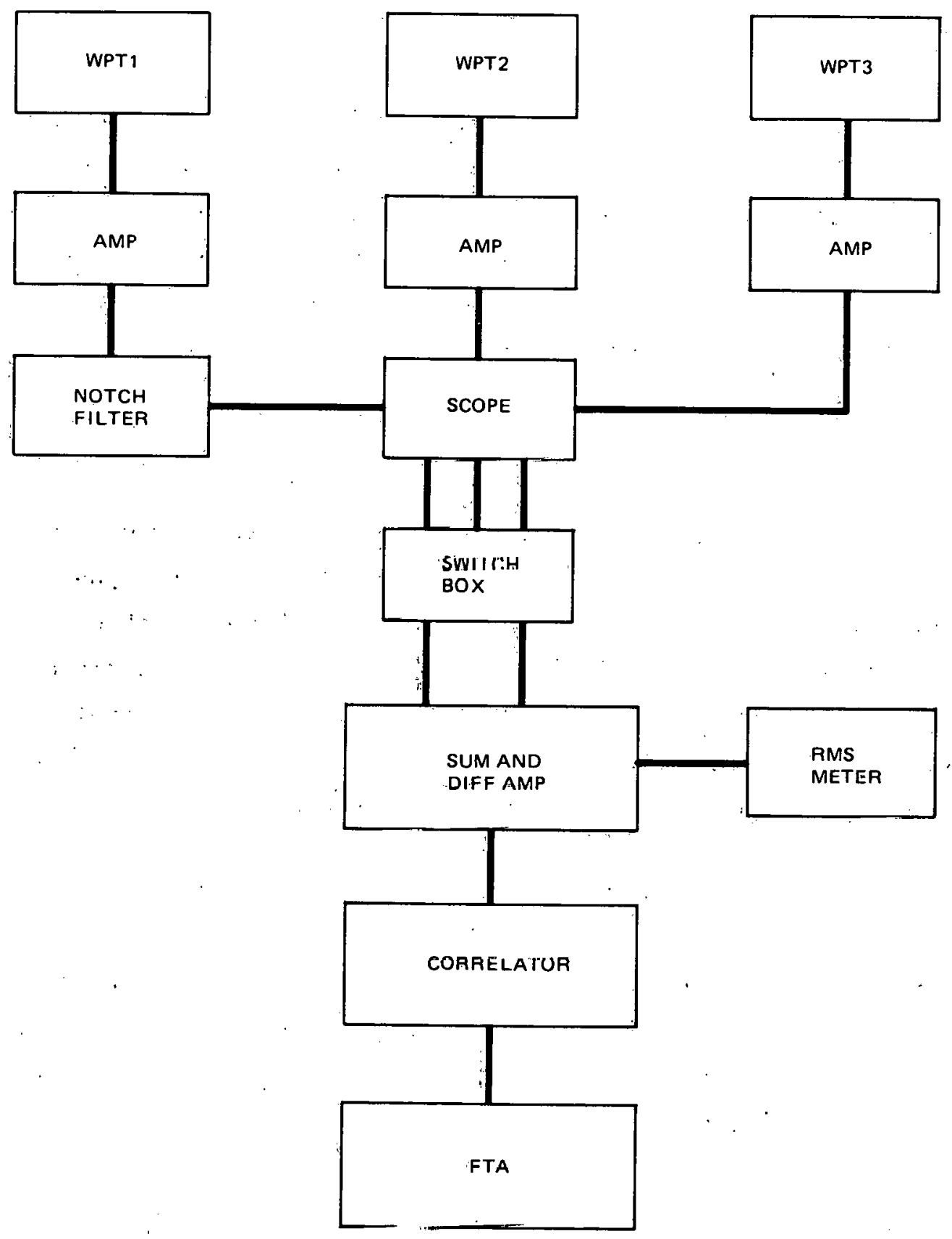

Figure 4-9. Block Diagram of the Wall Pressure Data Acquisition System 


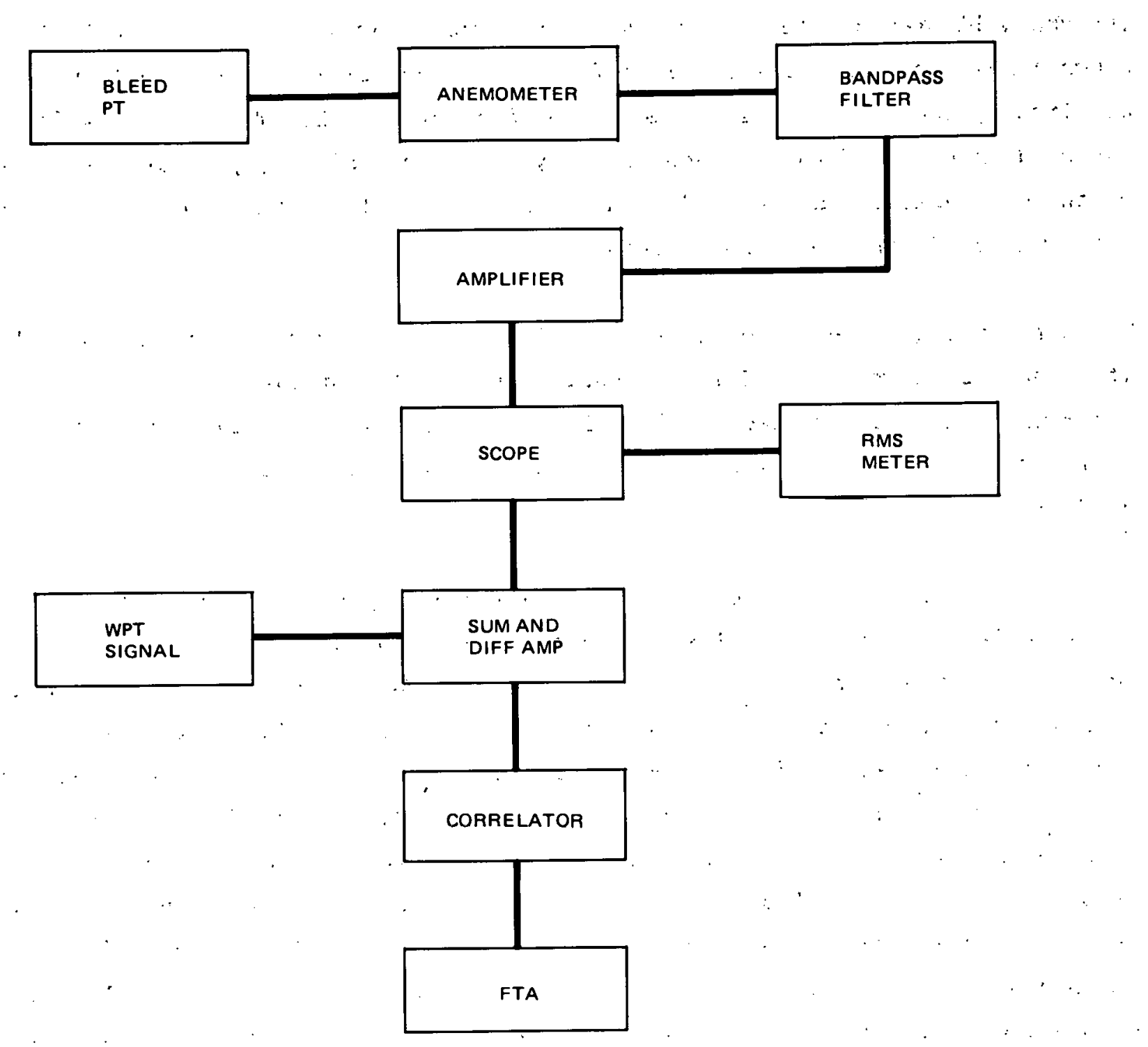

Figure 4-10. Block Diagram of the In-Stream Pressure Data Acquisition System

The initial single point measurements resulted in RMS levels that were significantly higher than the accepted values. It was found that the discrepancy could nnly he accounted for by the presence of a significant acoustic pressure field in the flow channel. The data aralysis outlined in Subsection 3.2 was then used to eliminate the superimposed acoustic pressure field from the total pressurc field. 
The RMS wall pressure level divided by the dynamic pressure $\left(q=1 / 2 \rho U_{\max }^{2}\right.$ ) is shown in Figure 4-11 for several different flow velocities. These data were acquired by use of the analysis method of Subsection 3.2. The maximum velocity was used in place of the average velocity as it most closely parallels the use of the mean velocity at large distances from the wall in the boundary layer work which was used for comparison purposes.

The data of Figure 4-11 show that the RMS level is lower on the inner wall than it is on the outer wal1. This is consistent with the predicted result of Schumann ${ }^{71}$ and the measured results of Brighton and Jones ${ }^{126}$ that the pressure is lower at the inner wall than at the outer wall with the difference increasing as $R_{1} / R_{2}$ decreases.

At large values of $U$, Figure 4-11 shows an increasing difference in the RMS pressure level at the two walls. It can not be assumed that this is an accurate portrayal as there is a significant uncertainty associated with each data point. This uncertainty as noted by the error bar in Figure 4-11 is due to the large acoustic contamination of the pressure field. The error bar of Figure 4-11 is an average uncertainty over the velocity range. This value is not constant as it is a function of the acoustic pressure level. For $\bar{U}=200 \mathrm{ft} / \mathrm{sec}$ the acoustic pressure level and the hydrostatic pressure level are approximately equal. However, for larger velocities the acoustic pressure level will increase faster than the hydrostatic pressure level as the acoustic prcosure is plupurtinnal to the mcan velocity Lu the 6th to 8 th power while the hydrostatic pressure is approximately proportional to the mean velocity to the 2 nd power. This will result in larger uncertainties at increasing mean flow velocities due to the increase in the signal rat1o of acoustic pressure to hydrostatic pressure. Part of this uncertainiy was the accuracy with which the acoustir signal could bc autracled out of the total signal. As the acoustic signal becomes dominant the subtraction process becomes one of subtracting two relatively large numbers to get a relatively small number. Thus any error in the subtraction process is increased when the acoustic pressure level is significantly larger than the hydrostatic pressure leve1. There is no data presented above $\bar{U}=250 \mathrm{ft} / \mathrm{sec}$ even though the syscem is capable of mean velocities as high as $400 \mathrm{ft} / \mathrm{sec}$ because the acoustic pressure signal becomes dominant and the accuracy of the signal subtraction becomes questionable. 


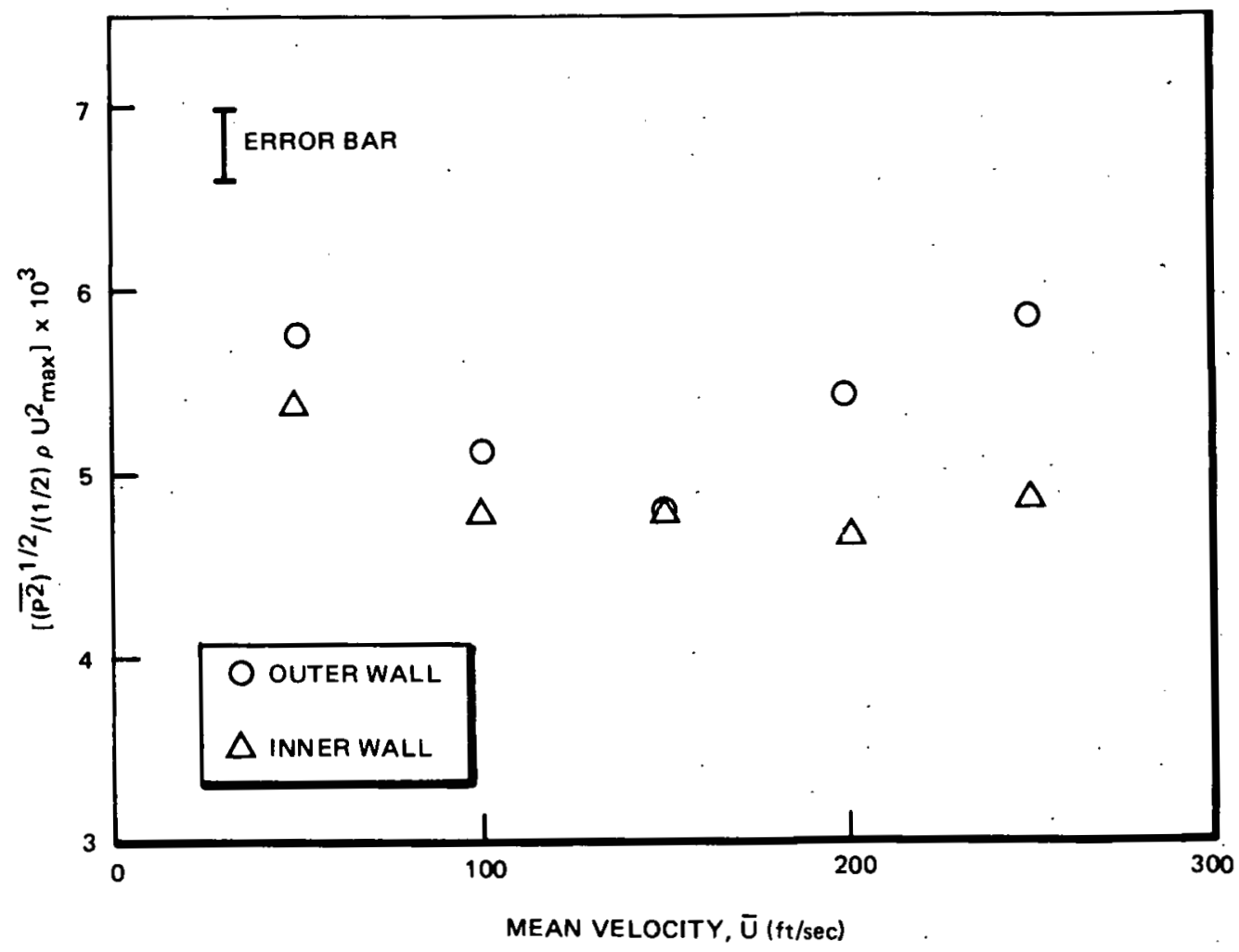

Figure 4-11. Wall Pressure Intensity Level as a Function of Mean Flow Velocity 
Measurement of the PSD of the acoustic pressure field is shown in Figure 4-12. This was done by cross-correlating the two outer wall pressure transducers which are assumed to be hydrodynamically uncorrelated and Fourier transforming the cross-correlation. A PSD of the signal from a single outer wall pressure transducer is also shown in Figure 4-12 for comparison purposes. The cross-spectra show peaks which correspond with peaks in the single sensor spectra which implies that these peaks are due to acoustic pressure fluctuations rather than hydrostatic pressure fluctuations. These peaks were observed to increase in magnitude with increasing mean flow velocity, but they did not shift in frequency with a change in mean flow velocity. This too implies that these peaks are acoustic in origin and probably caused by a Helmoltz resonation somewhere in the flow system. Axial separation and cross-correlation also showed that the convection velocity of these peaks was equivalent to the sonic velocity in air, which clearly implies that it is acoustic in nature rather than hydrodynamic.

The acoustic pressure field is seen to be mainly low frequency fluctuations of less than $400 \mathrm{~Hz}$ with spikes at $3.4 \mathrm{KHz}$ and $7.4 \mathrm{KHz}$. The source of the $3.4 \mathrm{KHz}$ and $7.4 \mathrm{KHz}$ acoustic noise could not be located. The lower frequency, broad band acoustic noise is thought to originate in the flow through the piping which leads to the flow development section.

In theory it would be possible to use the known acoustic PSD to obtain the hydrostatic pressure PSD from the signal of a single wall pressure transducer. However, in practice this approach proved difficult as it entails the subtraction of two large numbers which are nearly equal. The error associated with this approach was greater than the signal subtraction method which was used for all if the hydrostatic pressure fluctuation measurements in this investigation.' The cross-spectra demonstrate that the peaks in the single sensor data are due to the acoustic pressure field and that there is a large acoustic contribution to the FSU. In the low trcquency range.

The PSD of the wall pressure as measured with the signal subtraction analysis method is shown in Figure 4-13 for the inner wall, the outer wall and in-stream pressure transducer at $R^{*}=0.90$ for $\bar{U}=200 \mathrm{ft} / \mathrm{sec}$. The PSD, $F_{p p}(f)$, is nondimensionalized by the fluid density, $\rho$, the maximum velocity, $U_{\max }$, and the hydraulic diameter, $d_{h}$. This is the nondimensionalization used by Willmarth 


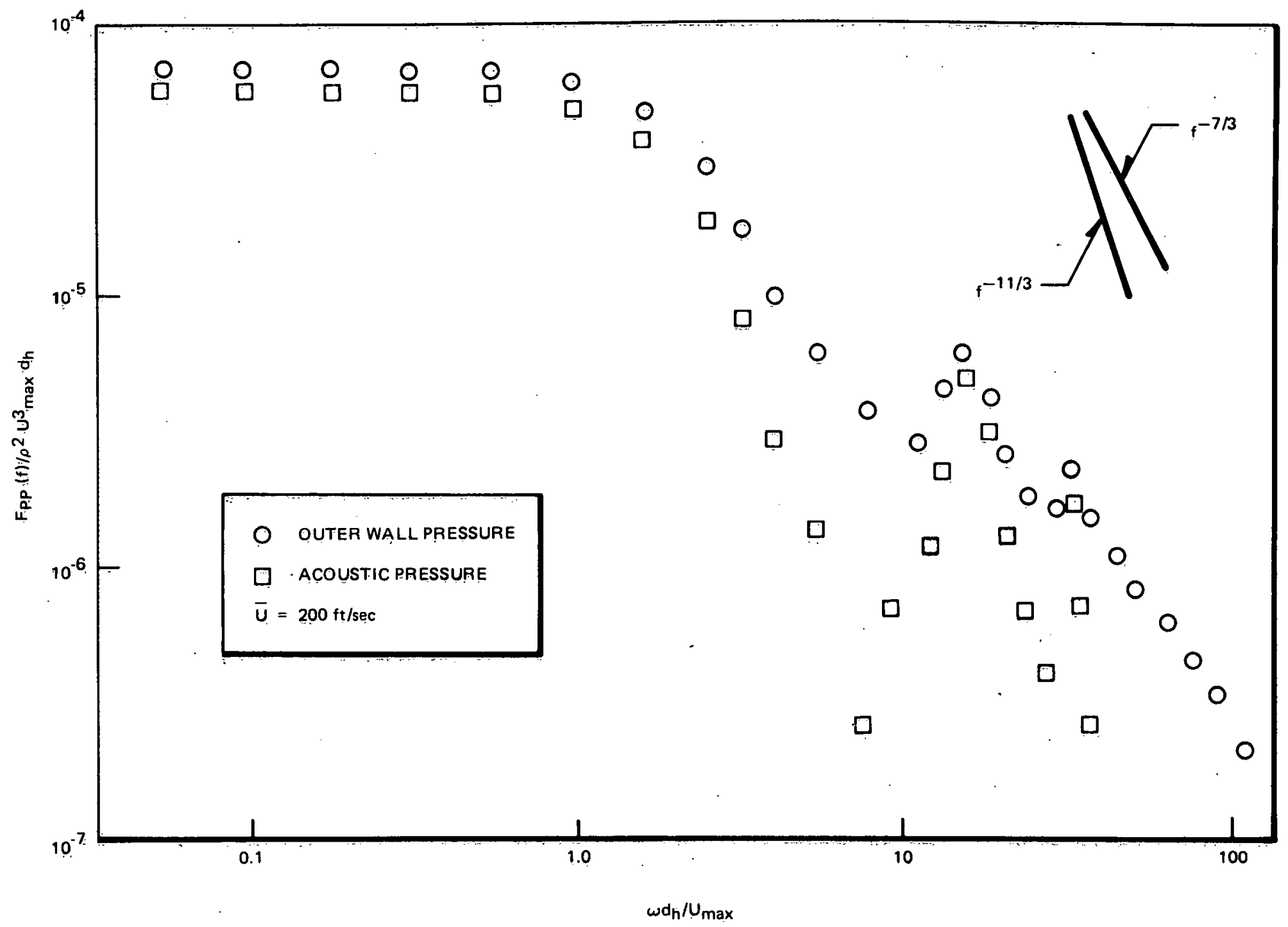

Figure 4-12: Power Spectral Density of a Single Wal1 Pressure Transducer and That of the Acoustic Pressure. Field 


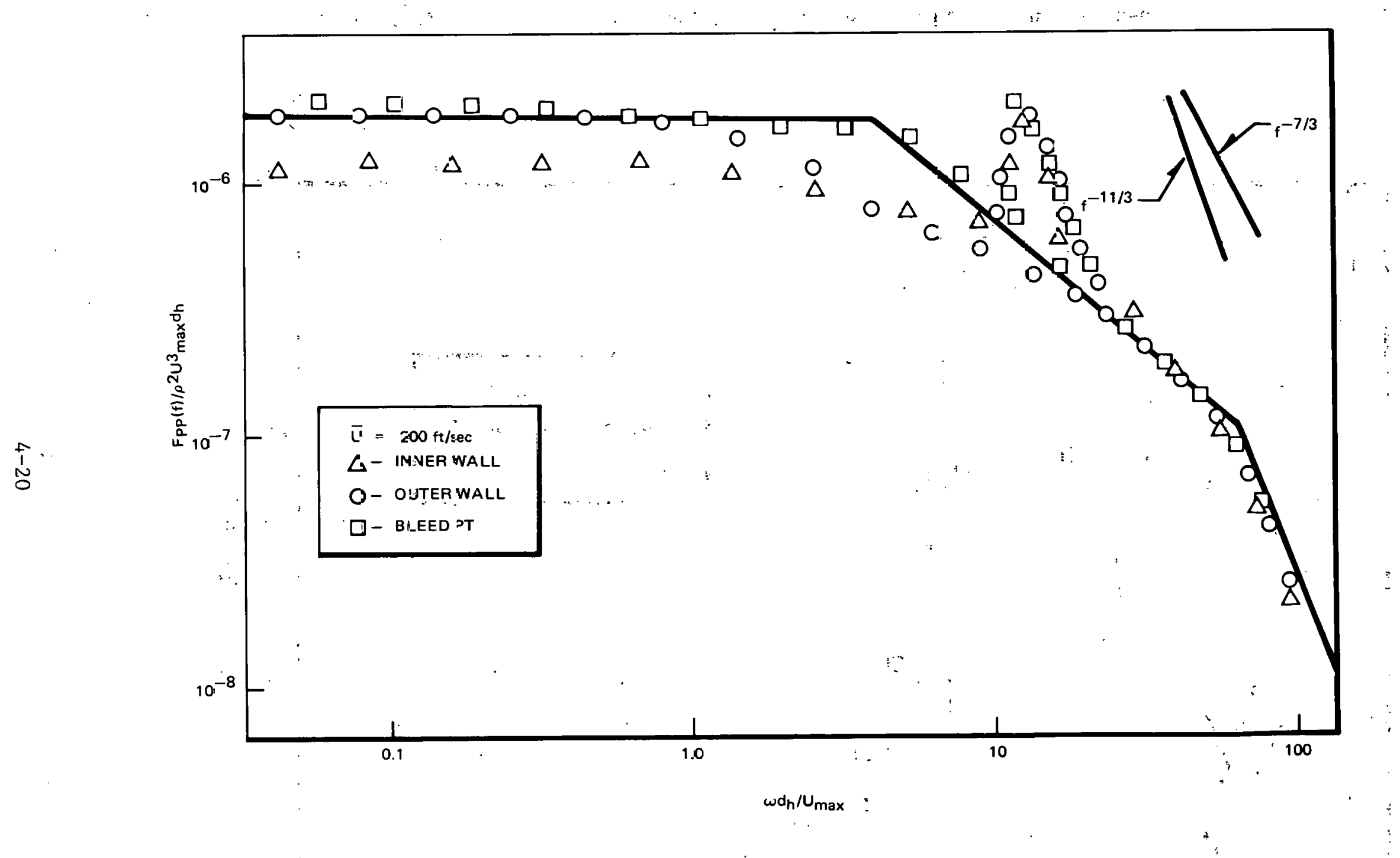

Figure 4-13. Power Spectral Density of the Hyjrostatic Wall Pressure and the In-Stream Pressure Showing the $3.4 \mathrm{KHz}$ Acoustic Peak Which. Could Not Be Electronically Renoved 
and Wooldridge 76 and is used here for comparison purposes and to show that the spectra adequately collapses for different flow velocities using these variables.

As can be seen in Figure 4-13, the peak at $3.4 \mathrm{KHz}$ is still in existence using the signal subtraction data analysis method. It is denoted by the solid symbols to infer that it exists from the data but is acoustic in origin as noted above. The signal subtraction would not eliminate this acoustic contribution to the sensor signal. The only explanations for this are either that the sensors were out of phase at this frequency or that the acoustic pressure wave was originating too close to the point of measurement to be of the same magnitude and phase at all radial and azimuthal locations in the axial plane of the wall pressure transducers. Since the phase response of the transducers was checked and no discrepancies were evident at this frequency it was assumed that the latter explanation was the case even though no source could be identified. The co-spectra measurements of Figure 4-12 and the axial separation cross correlation measurements were valuable in identifying this peak as an acoustic pressure source. The Fourier transform of the cross-correlation will yield the acoustic frequency components regardless of their phase relationship at the two transducers.

Since the $3.4 \mathrm{KHz}$ peak was due to acoustic contamination it was removed from all measurements by assuming the PSD of the hydrostatic pressure fluctuations was smooth and continuous across the frequency range of the acoustic resonance peak. All of the PSD's of the pressure measurements were corrected to eliminate this resonance peak.

The solid line drawn in Figure 4-13 is the assumed normalized PSD used by Chen and Wambsganss. ${ }^{30}$ This is a representative PSD taken from the data of Bakewe $11^{133}$ for a body of revolution in water flow, Clinch ${ }^{134}$ for water pipe flow, Willmarth and Wooldridge ${ }^{76}$ for air flow on a flat plate and Wambsganss and Zaleski ${ }^{73}$ for annular water flow. The agreement between the assumed PSD and that of the outer wall is quite good. However, the measured inner wall PSD is somewhat lower in the low frequency end of the PSD than the assumed PSD.

The PSD measured by the bleed pressure transducer of $R^{*}=0.90$ is in good agreement with the PSD measured at the outer wall $\left(\mathrm{R}^{*}=1.0\right)$. The edge of the displacement thickness on the outer wall was estimated to be at $R^{*}=0.86 \pm 0.05$. 
This agreement supports the use of this bleed pressure transducer for measurement of fluctuating static pressure in internal flows. Only one PSD measured with the bleed pressure transducer is shown, as the signal-to-noise ratio made it very difficult to obtain this information. "This probelm will be discussed in more detail later.

Figures 4-14 and 4-15 show the PSD of the pressure fluctuations at the inner and outer walls, respectively, for five different mean flow velocities. The PSD's collapse in magnitude for the nondimensionalization previously noted and in frequency for the Strouhal number based on the hydraulic diameter (i.e., $\left.\omega_{h} / U_{\text {max }}\right)$. The inncr wall PSD has a somewhat 1 nwer magnitule in the low fre= quency. range than the outer wall PSD. This could be due to the different curvatures of the two walls which yield different effective flow volumes. The point of maximum velocity lies nearer to the inner wall than to the outer wall. It would appear that the larger eddies would be of the order of the distance from the wall to the point of maximum velocity. If this is the case, there would be more larger scale, lower frequency, fluctuations near the outer wall than there would be near the inner wall.

The PSD of the inner wall has a much sharper cut-off frequency than that of the outer wall PSD. The cut-off frequency corresponds to $\mathrm{fd}_{\mathrm{h}} / \mathrm{U}_{\max }=2.5$ which is in agreement with the information given by $\operatorname{Shin}^{135}$. The outer wall pressure PSD also cuts-off near $\mathrm{fd}_{\mathrm{h}} / \mathrm{U}_{\max }=2.5$, but is not as pronounced as that of the inner wall.

Both the inner and outer wall pressure PSD's are very flat in the. low frequency end of the spectrum. The measurements of Figures 4-13, 4-14 and 4-15 are for frequencies as low as $10 \mathrm{~Hz}$ (high pass filters were set at $2 \mathrm{~Hz}$ ). At a mean velocity of $200 \mathrm{ft} / \mathrm{sec}$, this corresponis to $\omega_{\mathrm{h}} / \mathrm{U}_{\max }=0.036$ or $\delta / \mathrm{U}_{\max }=0.0025$ where $\delta$ is the displacement thickness.

Chen and Wambsganss ${ }^{30}$ point out that there is no dependable data below $\delta / U_{\text {max }}<0.07$. The reason for thic for the moat part: is the acoustic pressure field which severely contaminates the low frequency end of the PSD's. In many cases (i.e., Willmarth and Wooldridge ${ }^{76}$ ) the low frequency end is cut off with a high pass filter. 


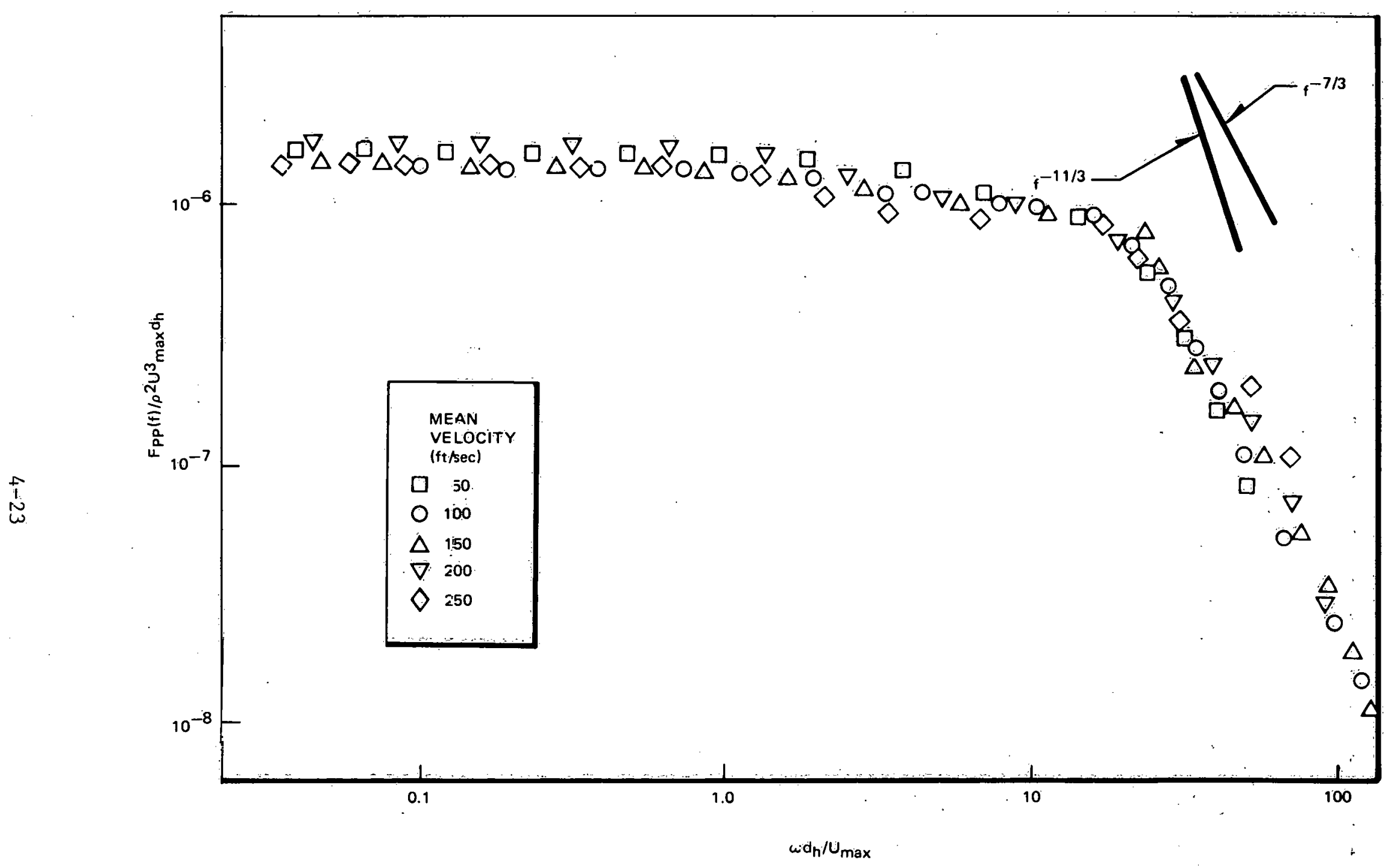

Figure 4-14. Power Spec-ral Density of the Hydrostatic Pressure Fluctuations at the Inner Wall for Several Different Mean Flow Velocities 


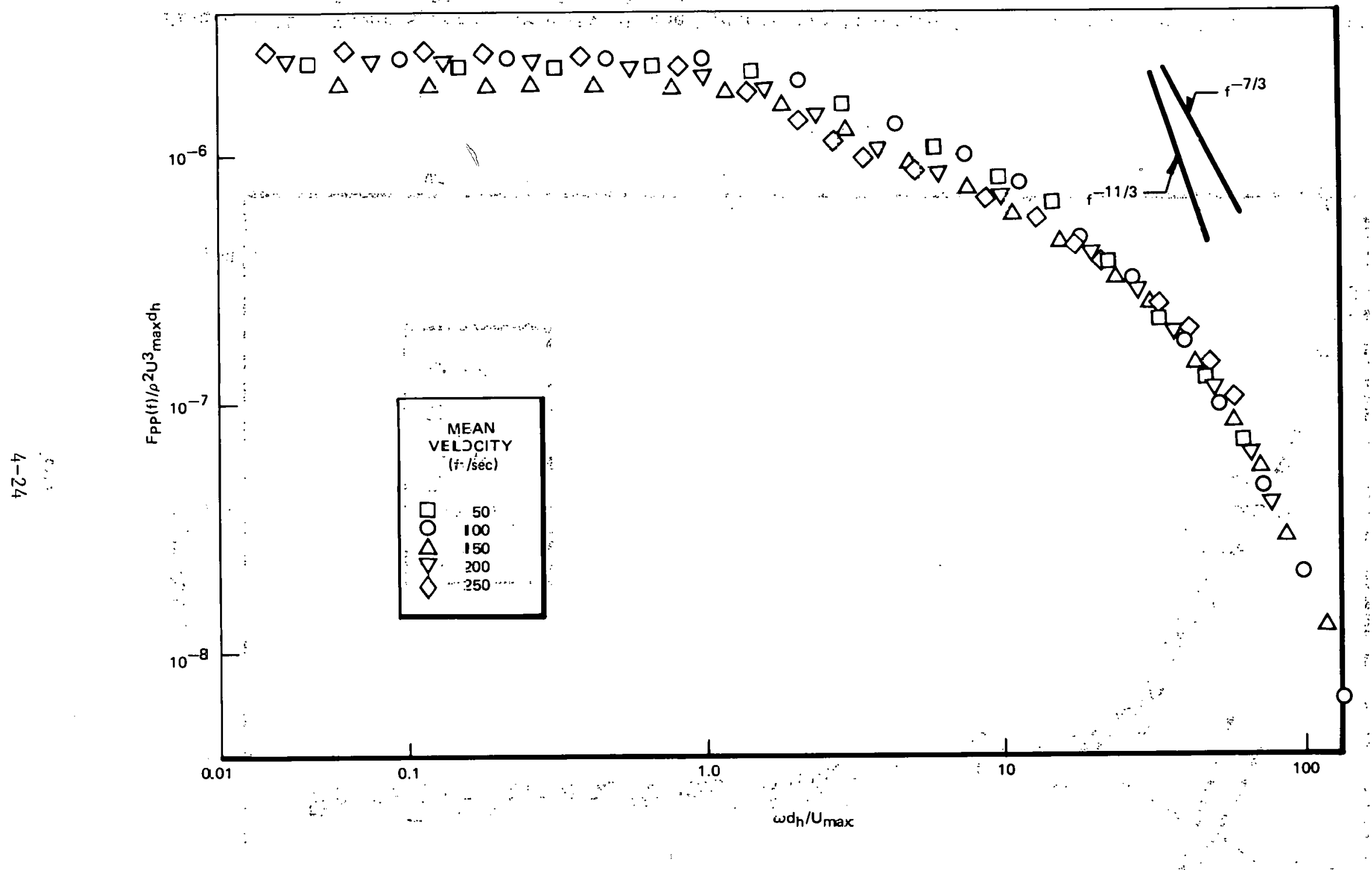

Figure 4-15. Power Spectral Density of the Hydrostatic Pressure F1jctuations at the Outer Wall for Several Differ 2 nt Mean Flow Velocities 
It is felt that the low frequency end of the PSD's of Figures 4-13, 4-14 and,4-15 accurately portray the frequency content of the hydrodynamic pressure fluctuations, as the system was designed specifically for the low frequency, measurements and there is a high confidence level in the data at low frequencies. Thus it would appear that the PSD's used for input to vibration models should be flat in the low frequency range, similar to the model used by Chen and Wambsganss. 30 This information would appear to be crucial to vibration models as this is the frequency range in which the fundamental frequencies of flow-induced vibrations occur. Since the origin of the driving mechanism lies in the fluid turbulence, it would seem that the frequency range of the turbulence which is of most importance to the excitation of flow-induced-vibrations would be of the same order of magnitude as that of the fundamental structural vibration.

Both the inner wall and outer wall pressure PSD's collapse at high frequencies. The slope is approximately $\mathrm{f}^{-9 / 3}$ above the cut-off frequency. This slope is consistent with the PSD information given by Chen and Wambsganss 30 which shows. the high frequency fluctuations dropping of at about $\mathrm{f}^{-9 / 3}$ for several different wall pressure measurements.

It should be noted that these PSD's have not been corrected for high frequency attenuation as discussed by Wilmarth ${ }^{82}$ and noted in Subsection 2.2. A correction would have the effect of increasing the high frequency end of the spectra which would shift the slope slightly towards $\mathrm{f}^{-7 / 3}$.

The measurement of the in-stream fluctuating static pressure was complicated by the lnw signal-to-noise ratio. The relatively low sensitivity of the bleed pressure transducers $\left(21.4 \mathrm{mV} / \mathrm{in}\right.$. $\mathrm{H}_{2} \mathrm{O}$ for $1412 \mathrm{G}$ and $25.1 \mathrm{mV} / \mathrm{in}$. $\mathrm{H}_{2} \mathrm{O}$ for $\left.1412 \mathrm{~J}\right)$, allowed the electronic noise to be a significant fraction of the total signal. This coupled, with the acoustic pressure contamination gave a significant uncertainty to the measurement of the fluctuating hydrostatic pressure field in the flow field region. The signal ratio for the measurement of the fluctuating hydrostatic pressure near the wall gave a mean square signal ratio of about 1:3:1 for the hydrostatic pressure signal to electronic noise signal to the acoustic pressure signal. This ratio was even worse near the center of the flow channel where the fluctuating hydrostatic pressure level was even lower. 
The RMS level of the in-stream fluctuating hydrostatic pressure was found with the analysis method outlined in Subsection 3.2. The acoustic noise level was assumed to be of equal magnitude at all points in the axial plane of the bleed pressure transducers. The resulting intensity profile of the fluctuating hydrostatic pressure field as a function of $R^{*}$ is shown in Figure 4-16. Two radial traverses are shown one with each bleed pressure transducer. The two traverses are also at two different azimuthal locations separated by 90 degrees. The relative uncertainties are shown in Figure 4-16 for transducer 1412J. This uncertainty would be approximately the same for transducer 1412G. The two traverses are in agreement within the uncertainty of the measurements.

Also showll in Figure 4-16 is the calculated RMS pressure profile of Schumann. ${ }^{71}$ The excellent agreement between the measured RMS pressure profile and the compucer generated protile is probably deceiving in as much as the computer generated RMS velocity profiles are significantly higher in magnitude than those which were experimentally measured. One would also expect Schumann's RMS pressure profile to be larger. in magnitude than that which was measured, but this is not the case. The discrepancy between the RMS velocity profiles and RMS pressure profile appears to be due to the computational models used to generate the turbulent fluid statistics, as all available.comparisons with published data show that the experimental data presented here are correct within the uncertainty of the measurements.

Considering the fact that Schumann's computational approach is the first effort specifically designed to generate information on the fluctuating static pressure field in channel flows and that the measurements presented here on the RMS profile of the fluctuating hydrostatic pressure are the first of their kind, there is a reasonably good agreement hetween the two. The gencral profiles whichi sliuw maximums near. the wall region similar to the RMS velocity profiles and minimums which occur near the point of maximum veinrity are in excellent agrccment.

The data obtained with the bleed pressure transducer are in good agreement with that of the wall pressure transducers as the bleed transducer is brought to the wa11. The PSD's of the two are in excellent agreement as shown in Figure 4-13, which lends a high level of confidence in the measurements taken with the bleed 


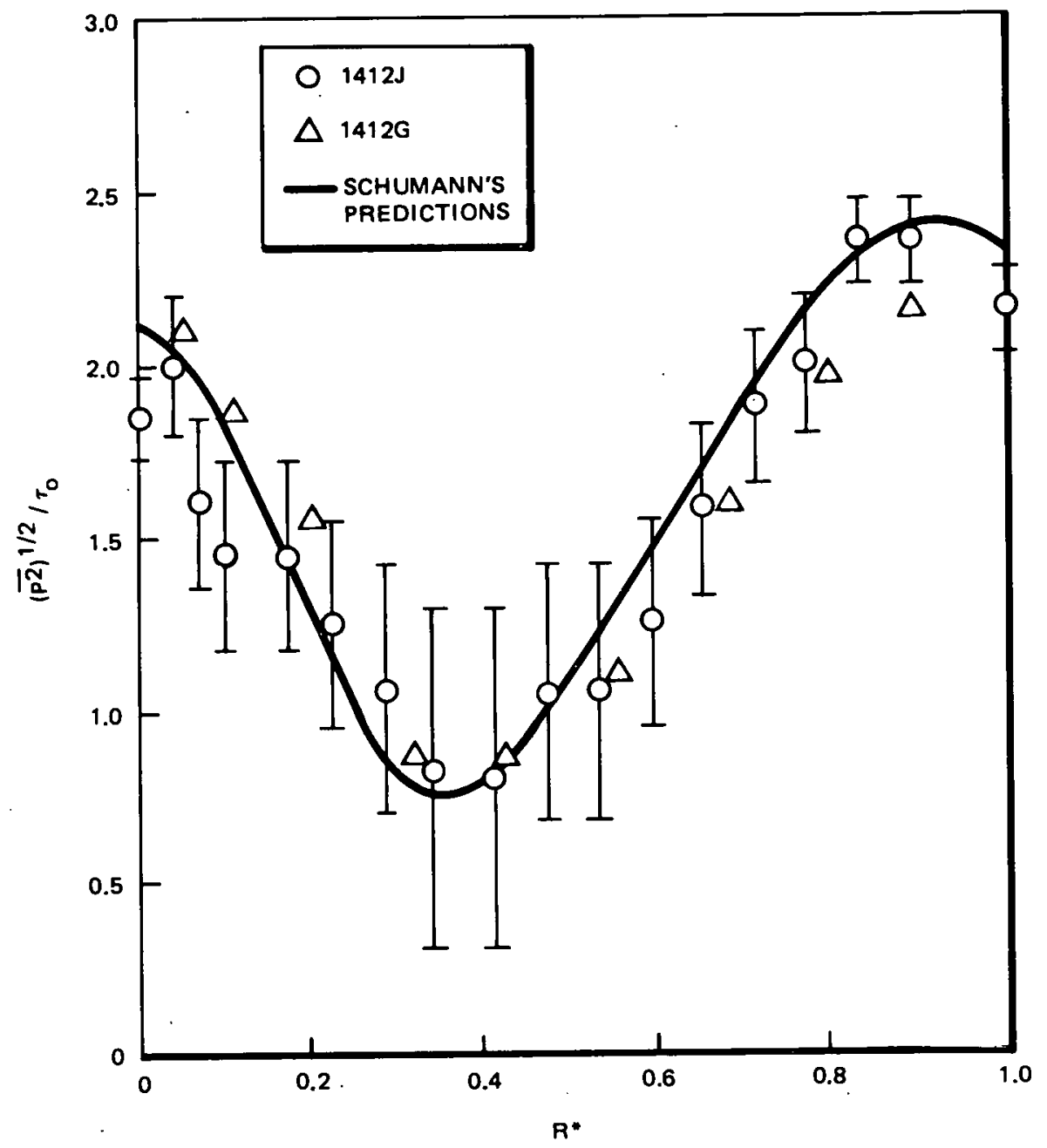

Figure 4-16. Profile of the In-Stream Hydrostatic Pressure Fluctuation Intensity Level

pressure transducer. Schumann's computer generated flow fields appear to generate information which is qualitatively correct, but is only an order of magnitude result in terms of the quantitative values. 


\section{TWO POINT MEASUREMENTS}

\subsection{PRESSURE-PRESSURE CORRELATIONS}

The two point pressure-pressure correlations were measured with the wall pressure transducers and the bleed pressure transducers or two bleed pressure transducers. The pressure-pressure correlations with the wall pressure transducers and the bleed pressure transducer allow for the investigation of the core flow pressure fluctuations influence on the wall pressure fluctuations. The two in-stream bleed pressure transducers were used to measure the correlation between pressure fluctuations in the flow field. The data analysis method used to measure the pressure-pressure correlations is outlined in Subsection 3.2.

The correlations between the in-stream bleed pressure transducer and the wall pressure transducers as a function of $R^{*}$ are shown in Figure 5-1 for the inner wall and Figure 5-2 for the outer wall. The two correlations are quite different for the two different walls with the inner wall correlation dropping off quickly and smoothly toward zero while the outer correlation has a significant plateau near the center of the flow channel. The difference between the two spatial correlations must be due to the different geometries of the two walls, but the exact cause is not fully explainable. The plateau of the outer wall correlation profile falls off to zero rapidly as the position of the in-stream bleed pressure transducer crosses the point of maximum velocity. This effect is not discernible on the inner wall correlation profile.

The integral spatial scale is defined as the integral of the spatial correlation over the full extent of the orthogonal direction. The correlation data was least squares fitted with arbitrary functions to give an adequate representation of the pressure-pressure correlation and these functions were then used to calculate the integral spatial scale. The fitted function for the inner wall correlation takes the form $R_{p p}\left(R^{*}\right)=0.0562\left(R^{*}+0.08\right)^{-1.14}$ and that of the outer wall correlation $R_{p p}\left(R^{*}\right) \stackrel{p p}{=} 0.22 \sin \left(\pi R^{*}\right)+R^{*}{ }^{7.02}$. These functions are shown in Figures $5-1$ and $5-2$ as the solid lines. 


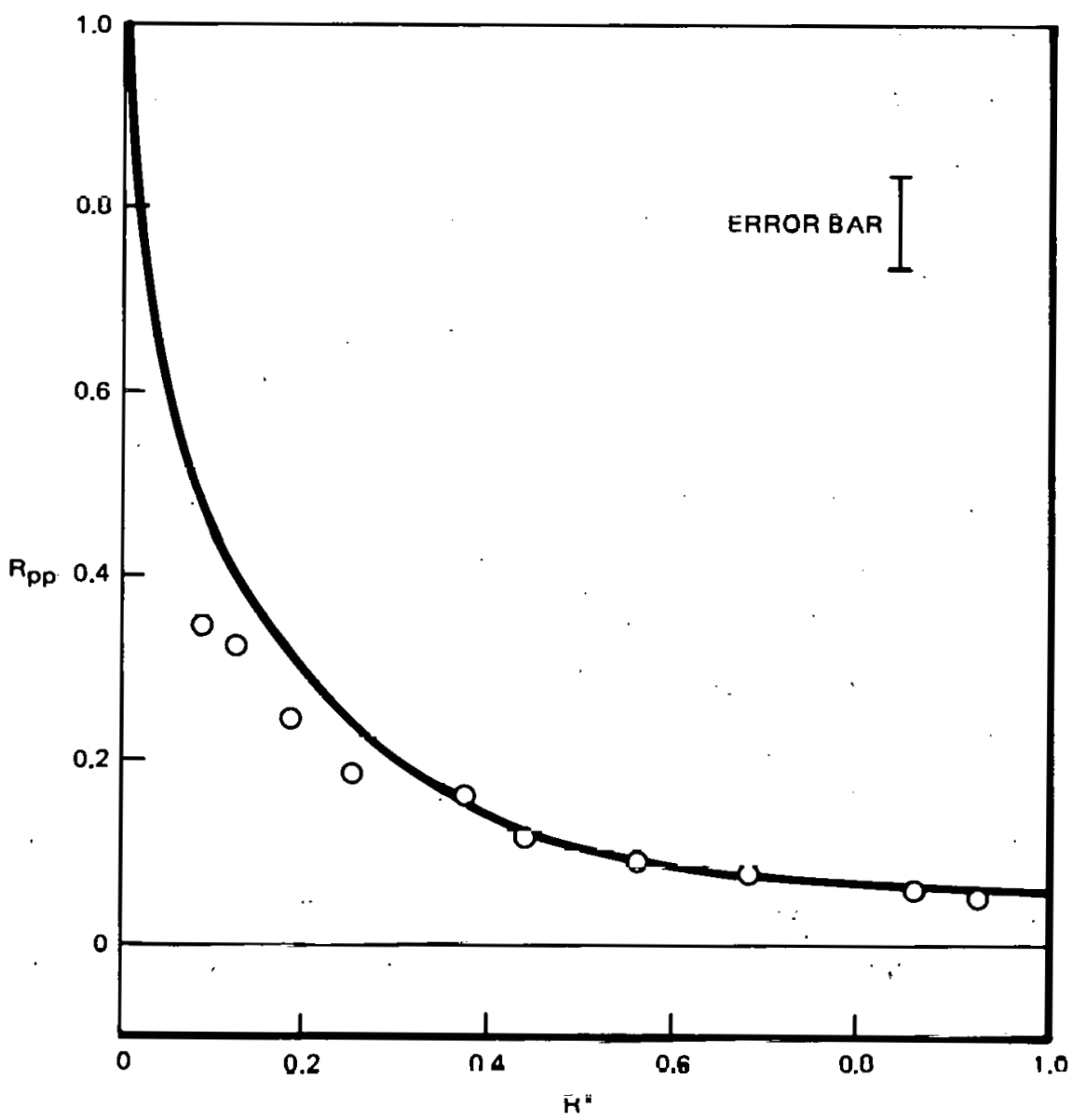

Figure 5-1. Correlation between the Inner Wall Pressure Fluctuations and the In-Stream Pressure Fluctuations as a Function of Radial Separation. 


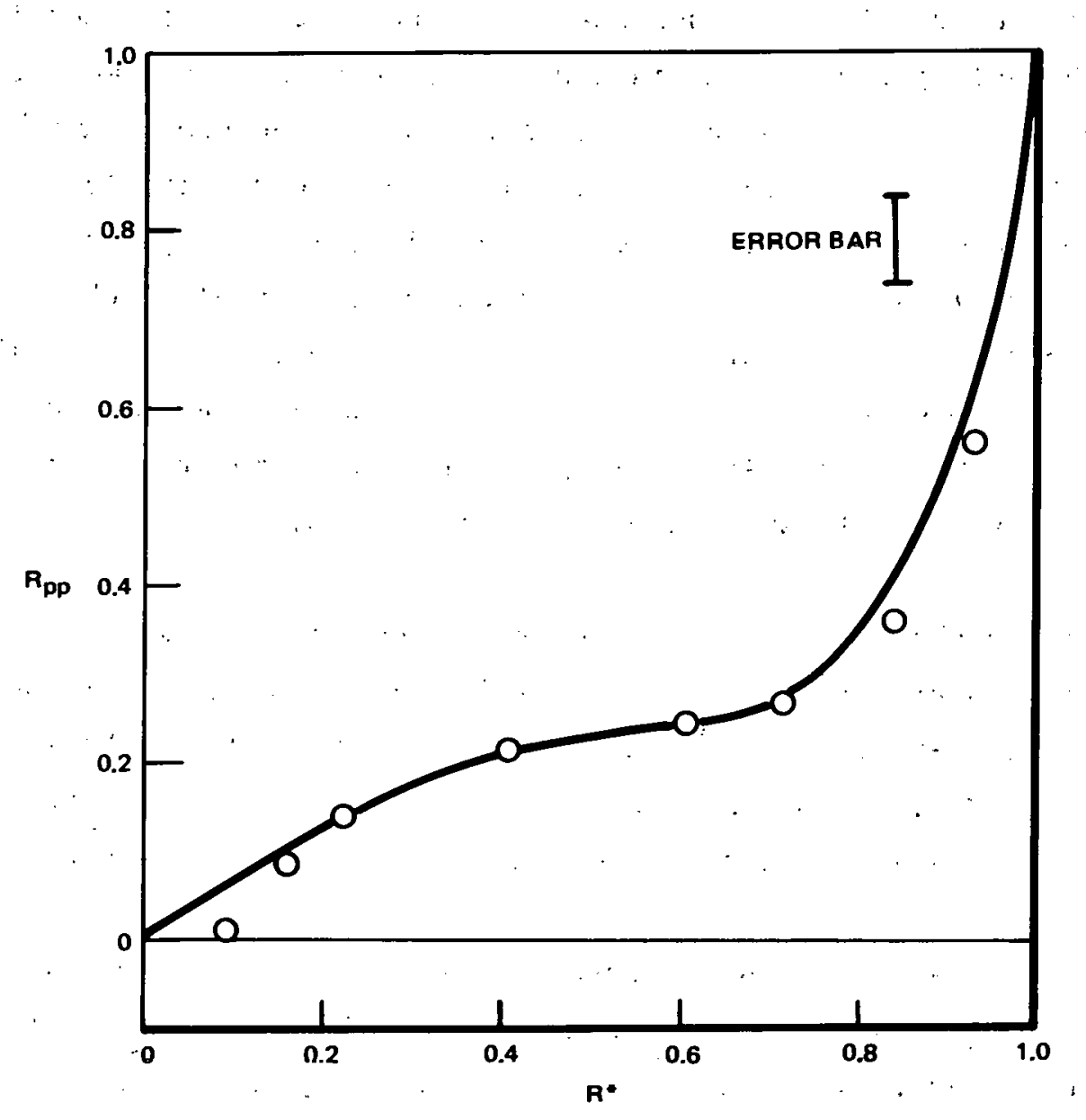

Figure 5-2. Correlation between the Outer Wall Pressure Fluctuations and the In-Stream Pressure Fluctuations as a Function of Radial Separation 
These functions give integral space scales of 0.175 for the inner wall correlation and 0.265 for the outer wall correlation. In both cases the fraction of the integral space scale that occurs within the displacement thickness is approximately $30 \%$. This would suggest that a significant amount of the wall pressure fluctuation is due to the core flow turbulence. No narrow frequency.band cross-correlations were performed, but such measurements may be useful in determining the in-stream turbulence structure which is correlating with the wall pressure. It is assumed at this time that most of this correlation is due to large scale, low frequency structure. Thus it is possible that the driving force for parallel flow-induced vibrations arises in the core region of turbulent channcl flows. Tlls would suggest that reduction of the large scale structure in the core region of turbulent channel flows would reduce the potential of flow-induced vihrations occurring. This could be achieved by reducing spacing between structures in the turbulent flow and thus reducing the maximum size of large scale structure or artificially altering the large scale structure with honey combs.

The aximuthal pressure-pressure correlations between the two in-stream bleed pressure transducers are shown in Figure 5-3 for $R^{*}=0.11$, Figure 5-4 for $R^{*}=0.25$, Figure 5-5 for $R^{*}=0.66$ and Figure 5-6 for $R^{*-0.89 . ~ M e a s u r e m e n t s ~}$ at $R^{*}<0.11$ or $R^{*}>0.89$ could not be performed as the wall-probe interaction in these regions altered the response of the blead procoure transducers.

The dzimuthal pressure-pressure correlations were fitted with simple exponential functions: With the exception of the azimuthal pressure-pressure correlation at $\mathrm{R}^{*}=0.11$, the exponential function gave a reasonibly good fit to the data. The fit is shown to improve with increasing values of $R^{*}$.

The azimuthal pressure-pressure correlation at $R^{\star}=0.11$ shows a minimum correlation at an angle of 120 degrees with increasing correlation from 120 degrees to 180 degrees. These data show a significant positive correlation between the pressure fluctuations occurring near the wall of the center rod for angular separations of 180 degrees. Azilluthal pressurepressure correlation measurements on the surface of a rod in a wind tunnel by Willmarth and Yang ${ }^{80}$ do not show a similar effect. However, Schumann's ${ }^{71}$ 


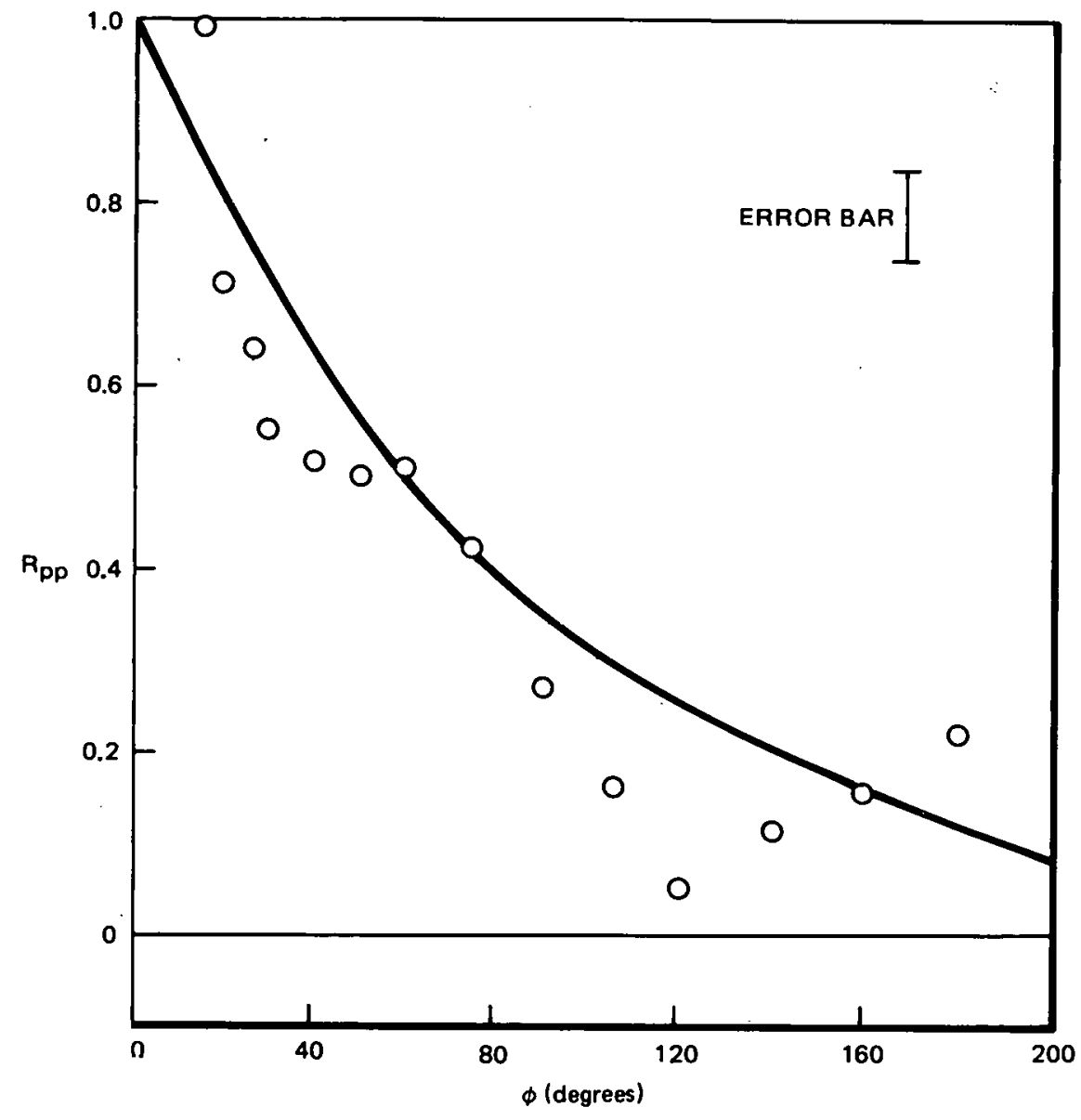

Figure 5-3. Azimuthal Pressure-Pressure Correlation between In-Stream Pıessure Traneducers at. $\mathrm{R}^{*}=0.11$

$$
\text { 5-5 }
$$




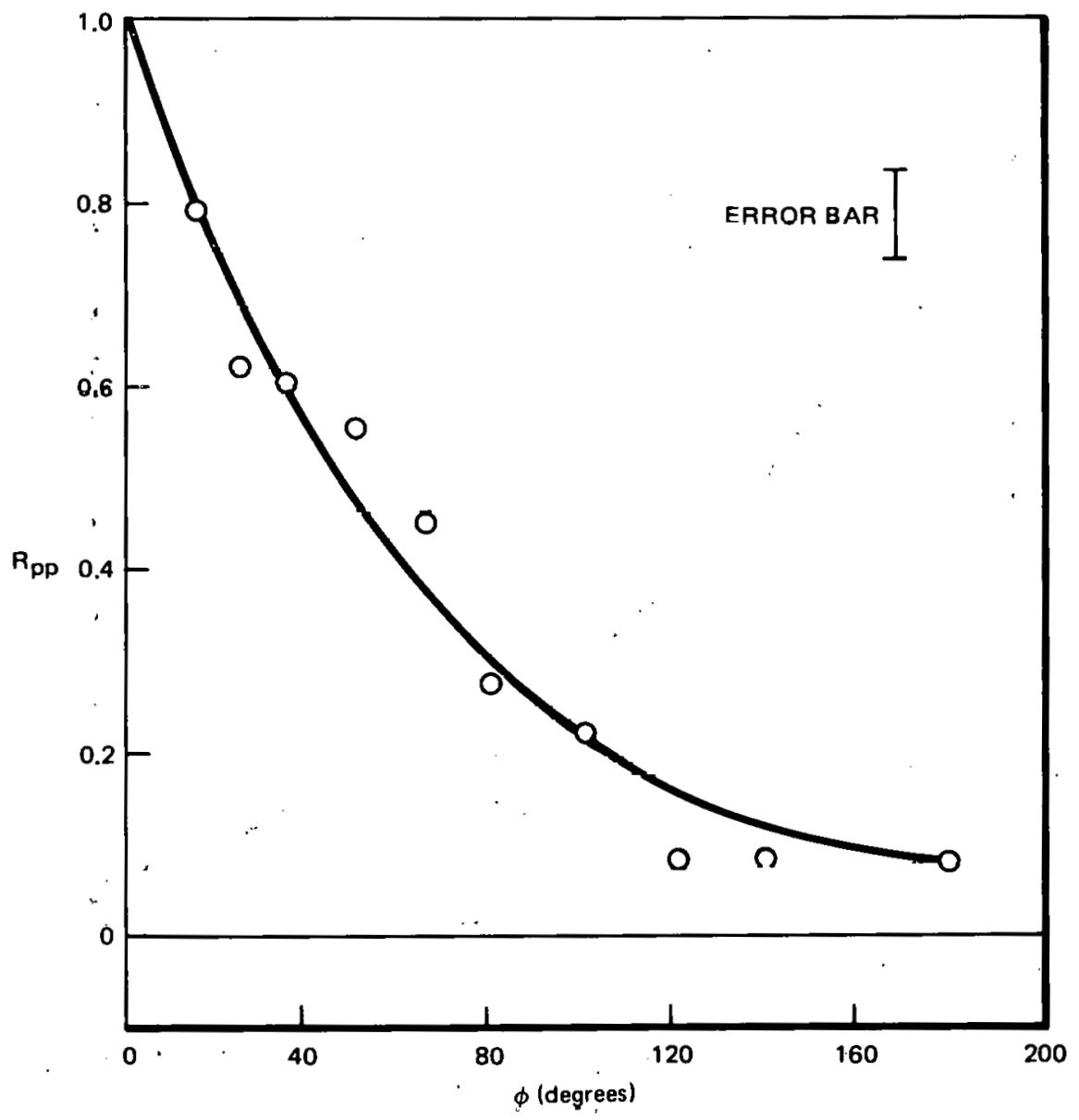

Figure. 5-4. Azimuthal-Pressure-Pressure Correlation between In-Stream Pressure Transducers at $\mathrm{R}^{*} * 0.25$. 


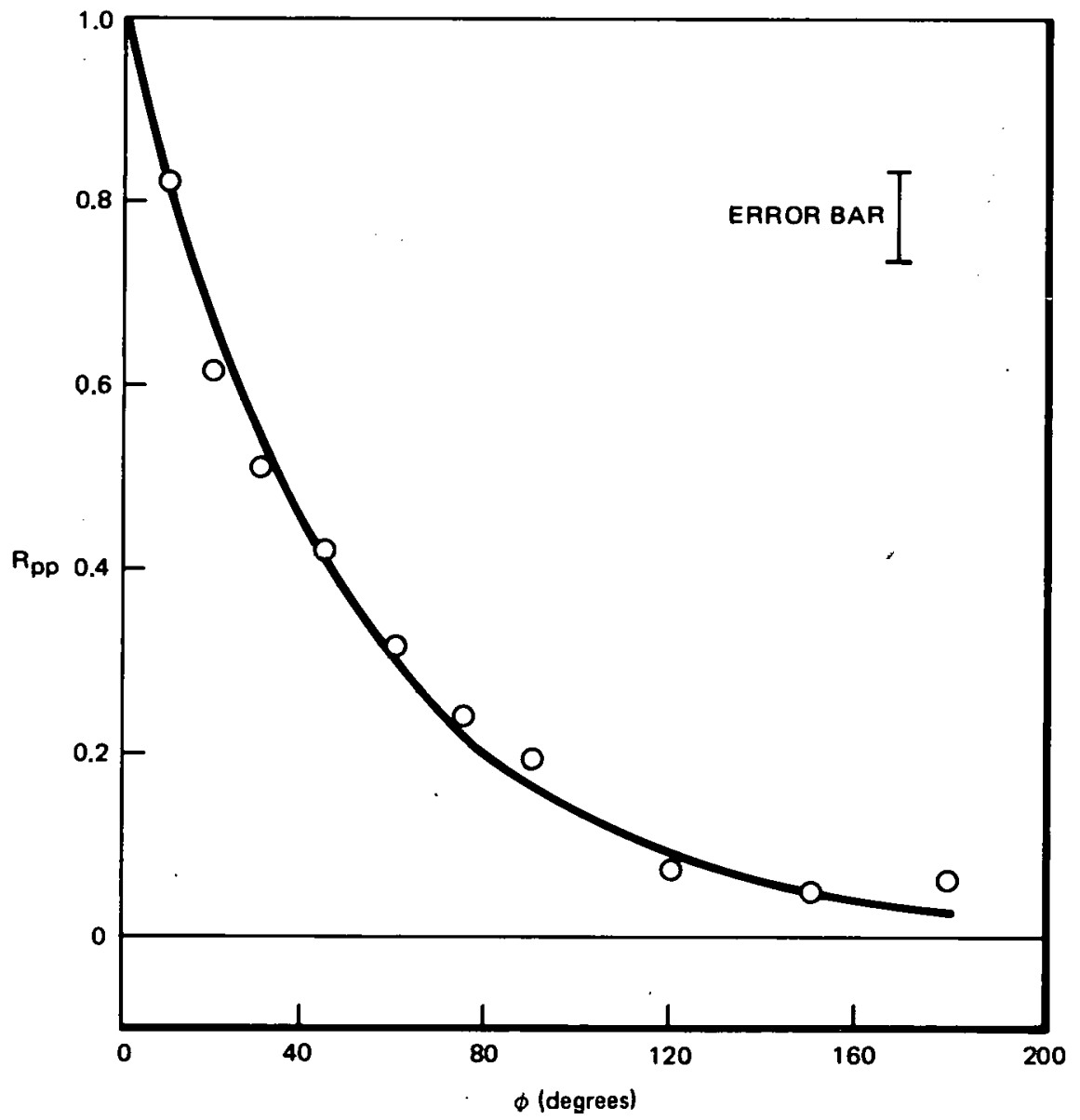

Figure 5-5. Azimuthal Pressure-Pressure Correlation between In-Stream Pressure 'I'ransduces's at $\mathrm{R}^{*}-0.66$ 


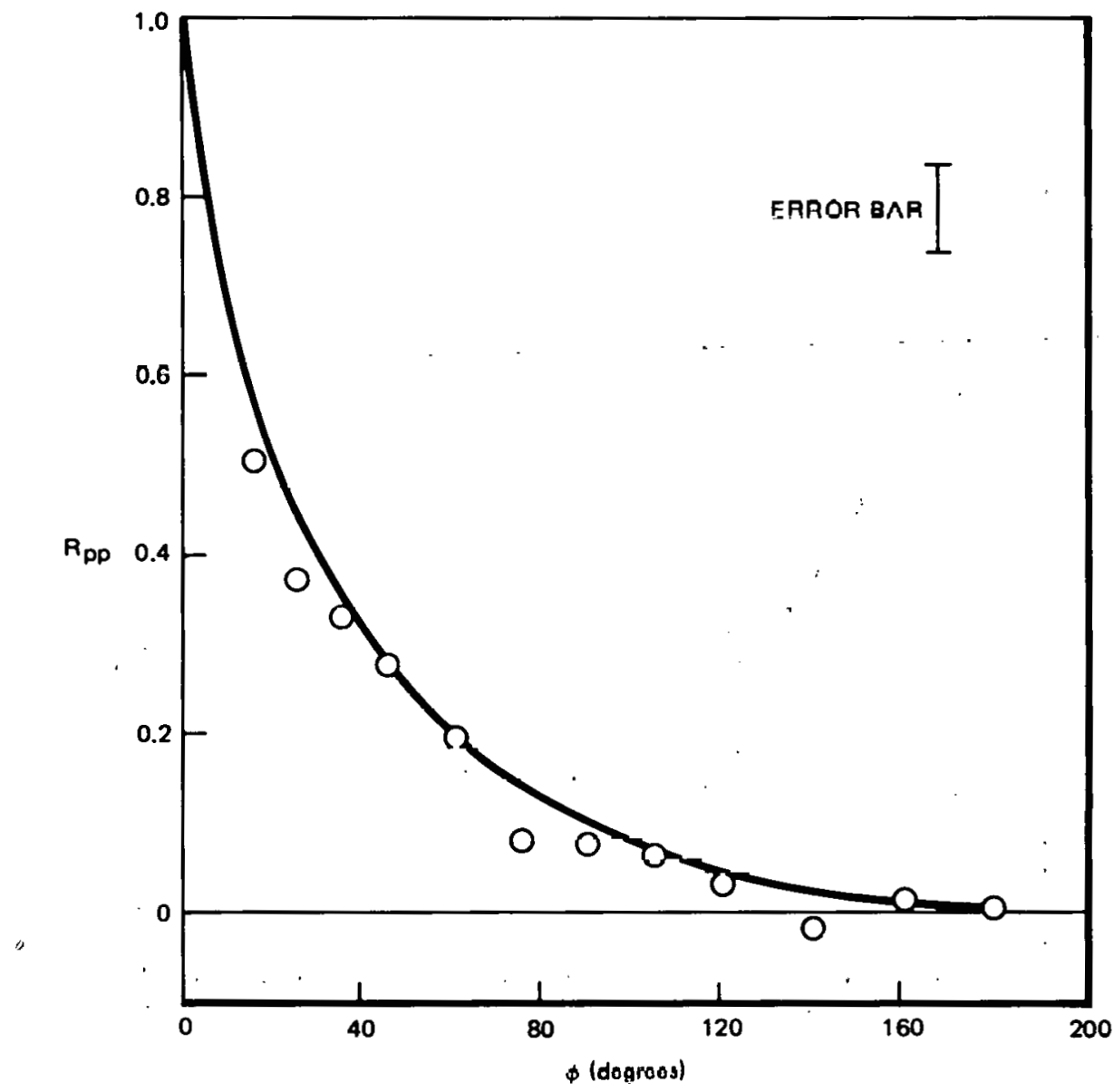

Figure 5-6. Azimuthal Pressure-Pressure Correlation between InStream Pressure Transducers at $R^{*}=0.89$ 
numerical simulation shows a dip in the correlation at the inner wall of the annular flow channel at about 120 degrees. The magnitude is significantly less than that shown in Figure 5-3 and Schumann implies that it may only be a statistical fluctuation. Schumann also shows that the correlation drops off much faster at the outer wall than it does at the inner wall, which is in agreement with the data presented here.

In comparing the azimuthal correlations at the walls, as calculated by Schumann ${ }^{71}$, with the azimuthal correlations in the flow field, it is seen that the wall correlations drop off much more quickly. This is not surprising in as much as the geometry is such that large scale structure can not exist to as great of an extent at the wall as it can in the flow field. The integral space scale for the azimuthal pressure-pressure correlations were taken from the exponential fit of the data. The least squares exponential curve fits were $\exp (-0.0116 \phi)$ for $R^{*}=0.11$, $\exp (-0.0150 \phi)$ for $R^{*}=0.25, \exp (0.0210 \phi)$ for $R^{*}=0.66$ and $\exp (-0.0263 \phi)$ for $R^{*}=0.89$. These functions result in integral space scales of 86 degrees at $R^{*}=0.11$ ( 0.51 inches), 67 degrees at $R^{*}=0.25$ ( 0.53 inches), 48 degrees at $R^{*}=0.66$ (0.67 inches) and 38 degrees at $R^{*}=0.89$ ( 0.65 1nches). This compares to approximately 15 degrees at $R^{*}=1.0$ ( 0.28 inches) and 30 degrees at $R^{*}=0.00$ (0.13 inches) for Schumann's data.

The data of Figures 5-3, 5-4, 5-5 and 5-6 can be collapsed for the core flow region $\left(0.11<R^{*}<0.89\right)$ by the function $R_{p p}(\phi)=\exp \left(-0.0275 \phi R^{*} 0.392\right)$. This function is not valid outside of the designated region as it gives correlations at $\mathrm{R}^{*}=0.00$ and $\mathrm{R}^{*}=1.00$ wh1ch are much different than those measured by Willmarth and Yang ${ }^{80}$ or predicted by Schumann ${ }^{71}$, [i.e. for $R^{*}=0.00$, $\mathrm{R}_{\mathrm{pp}}(\phi)=1.0$ for $\left.\left.\mathrm{a} 11 \phi\right)\right]$.

Figures 5-7 and 5-8 are correlations between the wall pressure fluctuations for azimuthal separation. Figure 5-7 is the azimuthal pressurepressure correlation between the Inner wall pressure transducer and the in-stream pressure transducer at $R^{*}=0.11$. Also shown in Figure $5-7$ is the azimuthal pressure-pressure correlation on the surface of a rod as measured by Willmarth and Yang ${ }^{80}$ and the numerical results of Schumann's work ${ }^{71}$ for 


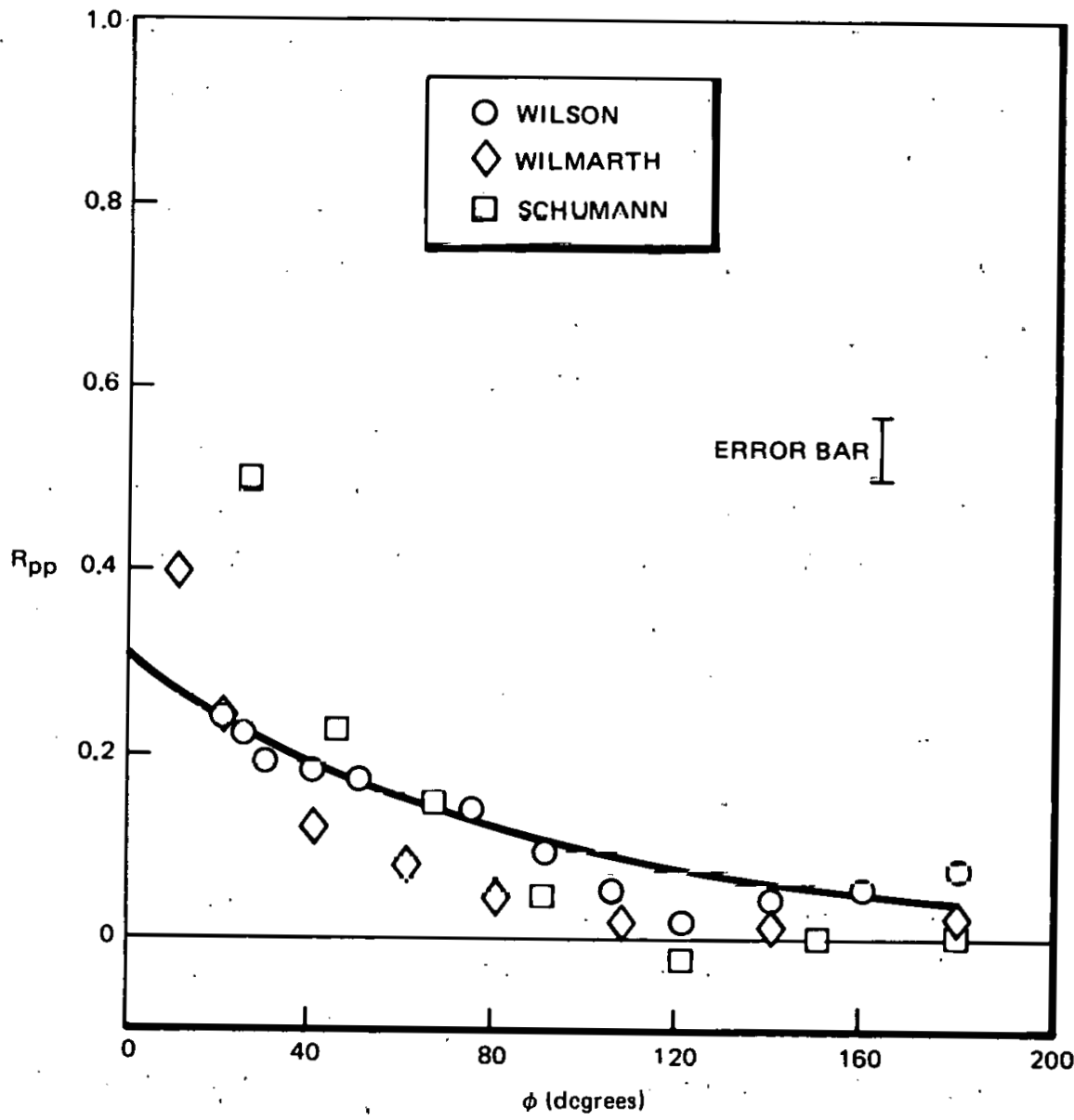

Figure 5-7. Azimuthal Pressure-Pressure Correlation between the In-Stream Bleed Pressure Transducer at $R^{*}=0.11$ and the Inner Wall Pressure Transducer 


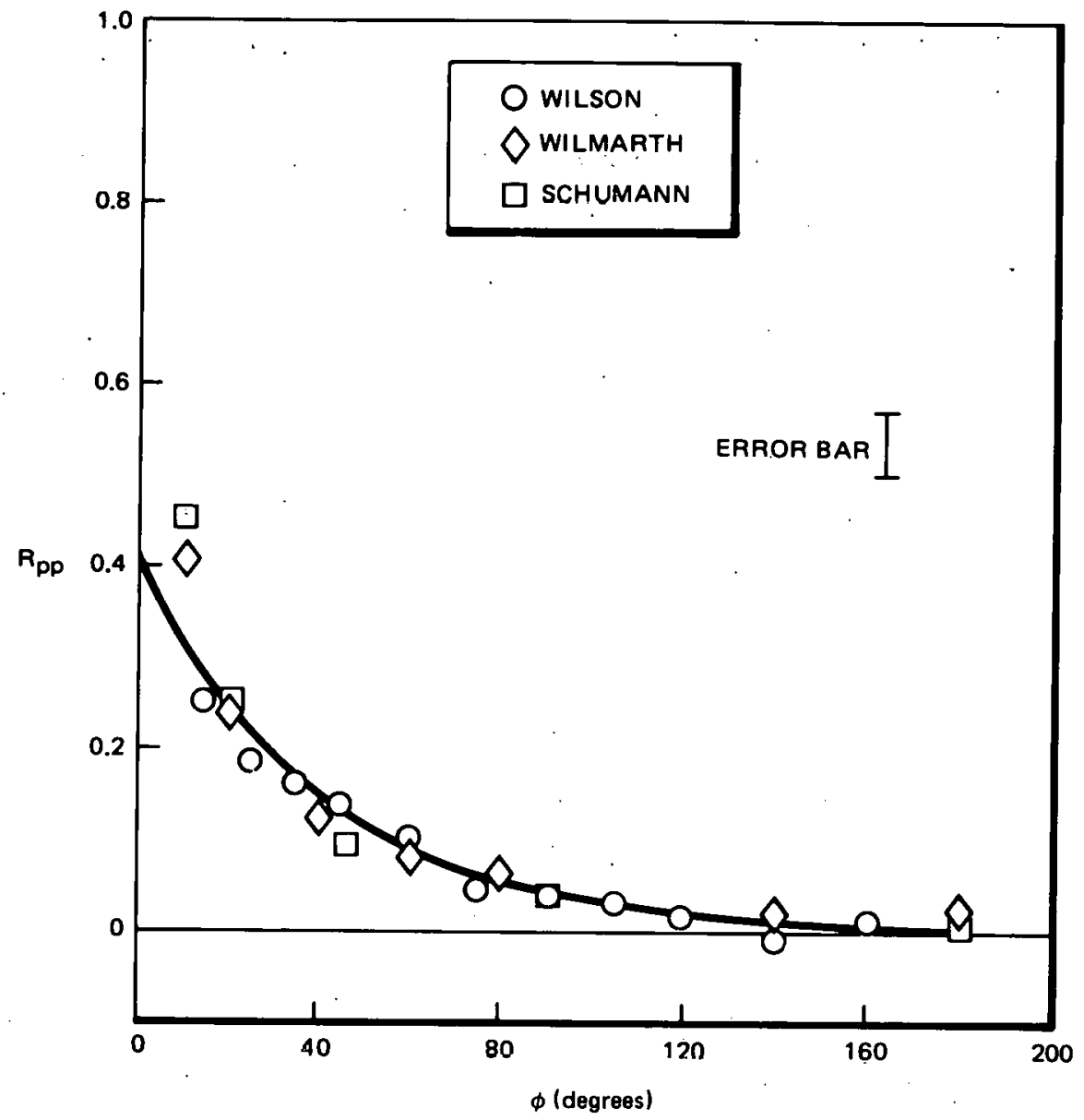

Figure 5-8. Azimuthal Pressure-Pressure Correlation between the In-Stream Bleed Pressure. Transducer at $\mathrm{R}^{*}=0.89$ and the Outer Wall Pressure Transducer 
the inner wall of an annular flow channel. The present data approaches 0.31 as $\phi$ goes to zero which is the value of the correlation at $R^{*}=0.11$ for the radial pressure-pressure correlation. The other data approach 1.00 as $\phi$ goes to zero as it should by definition of the cross-correlation. Thus there is a significant difference for small $\phi$ as the correlations being compared are significantly different. However, for $\phi>40$ degrees (0.16 in.) the correlations are in reasonable agreement. The general shape of the correlation is very similar to Schumann's data with a slight shift in the magnitude.

Similar correlations are shown in Figure 5-8 for the outer wall pressure transducer and the bleed pressure transducer at $\mathrm{R}^{\star}=0.89$. The same data by Willmarth and Yang ${ }^{108}$ as in Figure 5-7 is shown along with Schumann's 71 data for the outer wall. Again there aro the discrepdicles ar sma $\perp$ values of $\phi$ which are consistent with the way the data were acquired. There is very good agreement with Schumann's numerical data for $\phi>1$ degreac (0.19 in.). Willmarth and Yang's data appear to agree with the outer wall correlation better than with the inner wall correlations which are of the same wall surface curvature.

The agreement between the wall-wall pressure currelations and the wall-flow fleld correlations would seem to suggest that the pressure correlations at separation angles greater than 10 dogrces at the illler wa.1 and 10 degrcc3 at the outer wall are dup to tho corc peyiuin uf lle curbulent flow field. A large scale eddy in the core region can cause pressure oscillations at the wall which can be felt over fairly large separation distanres, which io to say there exists a correlation between these two pnints due to the origin of the pressure fluctuation being from the same large scale fluctuation nutside of the boundary layer. The agreement between the wal1-wal1 pressure correlations of Willmarth and Yang ${ }^{80}$ and Schumann ${ }^{71}$, and the data presented here suggeor that the correlation at separation distances of about 0.20 in. and greater is due to this effect as the correlations are shown to be approximately equal.

The pressure-pressure correlations for axial separation are shown in. Figure 5-9 for both the inner and outer walls. The inner wall correlation 


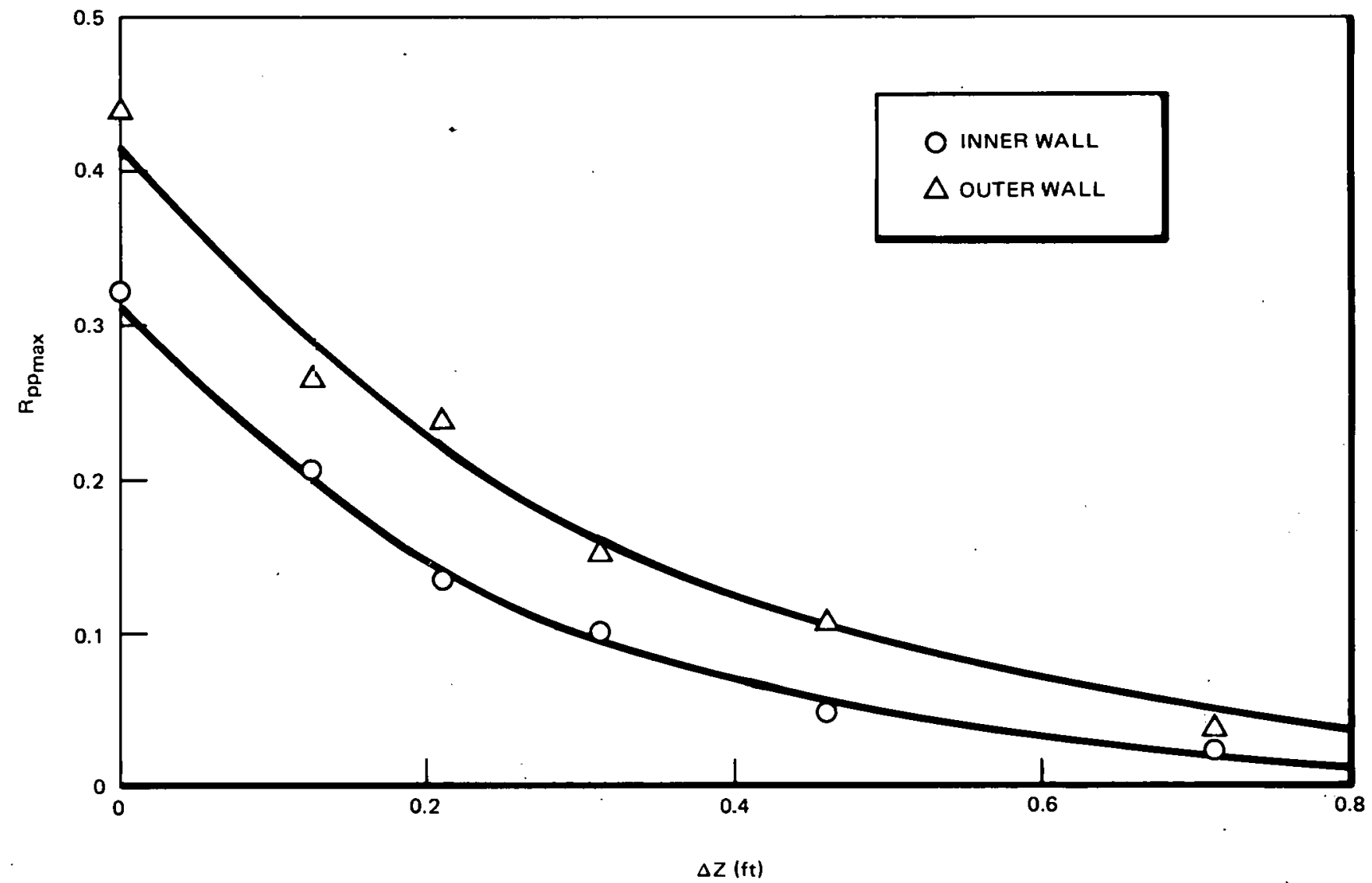

Figure 5-9. Axial Pressure-Pressure Correlation between the Wall Pressure Transducers and the Bleed Pressure Transducer at $R^{*}=0.11$ for the Inner Wall and $R^{*}=0.89$ for the Outer Wall 
was measured between the inner wall pressure transducer and the in-stream bleed pressure transducer at $R^{*}=0.11$. The correlation is least squares fitted with an exponential function which gives $R_{p p}(z)=0.31 \exp (-3.64 z)$ for the inner wall. The correlation goes to 0.31 for $z=0.0$ which is in agreement with the results of the azimuthal and radial correlations at $\mathrm{R}^{*}=0.11$ for the inner wall. This correlation function gives an integral length scale of 3.30 in.

The outer wall correlation which was measured between the outer wall pressure transducer and the in-stream bleed pressure transducer at $R^{*}=0.89$ is also shown in Figure 5-9. Tt too agrees witl the previuus measurements fur $R^{*}=0.89$ at $z=0.0$. The least squarco fitted exponential function is $R_{p p}(z)=0.41 \exp (-2.88 z)$ for the outer wall. This correlilinn function givcs an integral space scale of $4.17 \mathrm{in}$. This is consistent with the radial and azimuthal integral space scales which are also larger at the outer wall than at the inner wall.

Figure 5-10 is the delay time to maximum pressure-pressure correlation for different separation distances. The data are fitted wi.th a straight line which has a slope that yields a convection velocity of $2.04 \mathrm{t} / \mathrm{-} 5 \mathrm{ft} / \mathrm{sec}$. This convection velocity is approximately equal to the mean flow velocity of $200 \mathrm{ft} / \mathrm{sec}$. The ratio of the convection velocity to maximum velocity is $0.90+/ \ldots 0.02$ for these data. This is somewhat higher than the value for a plane boundary layer or a boundary layer on a circular cylinder which ranges from 0.50 to 0.85 depending on the separation distance ${ }^{80}$. The convection velocity increases for increasing coparation distances; thus the most accurate value for comparison is the largest value which is 0.85 as the separation for the data of Figure 3-10 also include some radial offset as it is the correlation between the wall prosare and Lhe in-stream pressure at $R^{*}=0.11$ and $R^{*}=0.90$, respectively. Grolibach ${ }^{1.36}$ has shown that the convection velocity to maximum velocity ratio for numerically simulated turbulent plane channel flows is 0.86 for low frequency turbulence. This too is in agreement with the boundary layer work cited by Willmarth and Yang $^{80}$ and slightly less than the value noted here. 


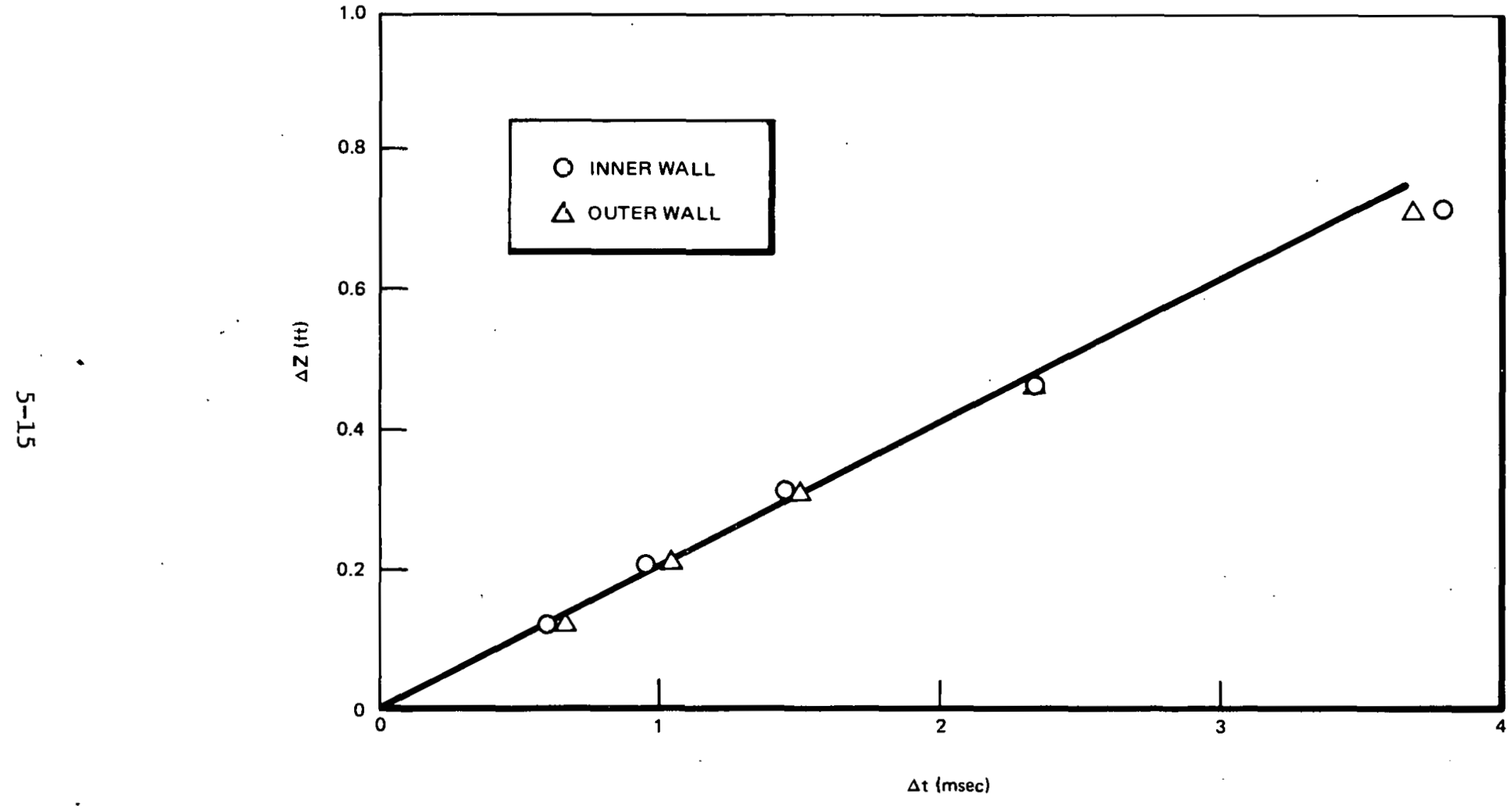

Figure 5-10. Delay Time to Maximum Pressure-Pressure Correlation for Axial Separation at Both Walls of the Flow Channel, which Results in a Convection Velocity, $U_{c}$, of $204+/-5 \mathrm{ft} / \mathrm{sec}$ 
The axial pressure-pressure correlation as a function of delay time also showed correlation peaks which could be attributed to acoustic noise propagation. The convection velocity of this correlation was equal to the speed of sound (1130 ft/sec) plus the mean fluid velocity (200 ft/sec). This correlation proves to be useful in identifying acoustic noise propagation as its convection velocity is well defined and unique to the acoustic pressure field.

The correlation between the wall pressure and the in-stream fluctuations can be approximated by combining the correlations of each of the three orthogonal directions. The resulting functional form for the inner wall is

$$
R_{p p}\left(R^{*}, z, \phi\right)=0.0562\left(R^{*}+0.08\right)^{-1.14} e^{-3.64 z} e^{-0.0275 \phi R^{*} 0.392}
$$

for $0.11<R^{*}<0.89$. The equivalent function for the outer wall is

$$
R_{\rho P}\left(R^{*}, z, \phi\right)=\left(0.22 \sin \pi R^{*}+R^{*} 7.02\right) e^{-2.88 z} e^{-0.0275 \phi R} 0.392
$$

for $0.11<R^{*}<0.89$. These correlation functions were obtained by directly multiplying the correlations from cach of the orthogonal directions together. It is the best estimate for the correlation between the flow field pressure fluctuations at any point in the core region of the flow field and the wall pressure fluctuations. More data for simultaneous separation in two or three directions is required to accuralely map the pressure-pressure correlation for the complete core flow region. No attampt has been made co universally apply these correlations to annular flow channels,

The resulting wall pressure-in-stream pressure correlations show that a significant correlation exists beyond the boundary layer region. The fluctuations which are correlating are believed to be large scale structure of relatively low frequency. It would appear that the frequency of these fluctuations would be of the same order of magnitude as the resultant flow 
induced vibrations. This would suggest that much of the driving force in channel flows may be due to the turbulence beyond the boundary layer region. It is certain that a complete picture of the fluid wall interaction can not be acquired by looking only at the wall pressure fluctuations. More detailed. pressure-pressure correlations, including narrow frequency band correlations, may be useful in interpreting the core flow turbulence effect on the wall pressure fluctuations.

\subsection{PRESSURE-VELOCITY CORRELATIONS}

The pressure-velocity correlations were measured with the wall pressure transducers and the cross-wire velocity probe. This made it possible to investigate the correlations between the hydrostatic wall pressures and the turbulent velocity components in the axial and radial directions. These correlations can provide some information on the interrelationship between the velocity field structure in the core region of the flow and the fluctuating hydrostatic wall pressure. The data analysis method used to measure the pressurevelocity correlations is outlined in Subsection 3.3.

In all of the radial and azimuthal pressure-velocity correlations there was a $0.1-i n$. axial offset between the center of the wall pressure transducers and the center of the $\mathrm{X}$-probe with the $\mathrm{X}$-probe being located upstream with respect to the wall pressure transducers. The pressure-velocity correlations, measured as a function of axial separation, included a 0.09-in. radial offset (with the $\mathrm{X}$-probe being moved downstream with respect to the wall pressure transducers). 'lhe axlal separation distances are noted as mounting port locations for the $\mathrm{X}$-probe which were shown in Figure 2-2. The actual separation distances were $-0.10 \mathrm{in}$. for position $2,0.90 \mathrm{in}$. for position $4,2.15$ in. for position $6,3.90 \mathrm{in}$. for position 8 and $6.90 \mathrm{in}$. for position 10 .

The defined geometry orientation for the pressure-velocity correlation is shown in Figure 2-5. The force exerted on an area of the wall by the fluid is given as ${ }^{137}$

$$
\overrightarrow{\mathrm{F}}=-\int_{\mathrm{A}} \hat{\mathrm{n} P \mathrm{dA}},
$$


where $\vec{F}$ is the force, $\hat{n}$ is the unit vector directed outward from the surface area into the fluid, $P$ is the average pressure of $\mathrm{dA}$ and $\mathrm{dA}$ is the surface area. The normal force on the surface area can be found by taking the scalar dot product of the force, $\vec{F}$, in the direction perpendicular to the wall surfaces. For the outer wall surface area the scalar dot product of $\overrightarrow{\mathrm{F}}$ with the unit vector. $\hat{r}$ in the radial direction, gives

$$
\mathrm{F}_{\mathrm{N}}=-\int_{\mathrm{A}} \hat{\mathrm{r}} \cdot \hat{\mathrm{n} P d A} \quad
$$

The scalar dot product of the radial unit vector with the outward drawn normal unit vector is equal to the cosine of the angle between the two vectors. For the suter wall this angle $1518 \mathrm{U}$ degrees, which gives a value of -1 . Thus the normal force on the surface area of the outer wall due to the fluid is

$$
\mathrm{F}_{\mathrm{N}}=\int_{\mathrm{A}} \mathrm{PdA}
$$

A similar analysis of the surface area on the inner wall defines the nurmal force as that given by Equation (5-4). However, on the inner wall the angle betwcen the unit vector in the radial directiun and the outward drawn normal is 0 degrees. This gives $\hat{r} \cdot \hat{n}=1$ for the inner wall and the normal force on the inner wall is given as

$$
\mathrm{F}_{\mathrm{N}_{\perp}}=-\int_{\mathrm{A}} \underline{\mathrm{PdA}} .
$$

The results of Equations (5-5) and (5-6) show that the correlations between the inner wall normal force (pressure) and the fluctuating velocity field. are of opposite sign with respect to those of the outer wall. This reșult . was experimentally observed and all inner wall pressure-velocity correlations have been multiplied by -1 to facilitate in the comparison of the results with those of the outer wall. 
Both the X-probe and the bleed pressure transducer were radially off-set 0.09 in. This value was selected for each sensor on the basis of the minimum radial offset which would give no perceivable influence on the wall pressure transducer signal. It was also experimentally verified that the acoustic pressure field was not affecting the pressure-velocity correlation as no perceivable correlation existed between the wall pressure signal and either velocity signal at large angular separations.

The two-point pressure-velocity cross correlation as a function of delay time and axial separation are shown in Figure 5-1 for the inner wall pressure and axial velocity; in Figure 5-12 for the outer wall pressure and axial velocity; in Figure 5-13 for the inner wall pressure and the radial velocity; and in Figure 5-14 for the outer wall pressure and the radial velocity.

The general shape of the correlation between the axial velocity component and the wall pressure is similar to that measured by Serafini ${ }^{72}$ in a plane boundary layer. However, the shape is quite different than the asymmetric correlations measured by Willmarth and Wooldridge ${ }^{77}$ in a thick boundary layer with zero longitudinal pressure gradient. The reason for this disparity between the results of Serafini and those of Willmarth and Wooldridge is not obvious but may be due to different experimental flow environments. In the case of Willmarth and Wooldridge, high intensity acoustic noise forced them to eliminate all information below $100 \mathrm{~Hz}$. It would appear that the correlation between pressure and velocity at this frequency would be fairly strong.

The Inluei wall correlation shown in Figure 5-11 shows a secondary peak or shoulder which dies away with increasing separation distance. This peak is not found on the correlation of the outer wall pressure with the axial velocity component. It may be due to the structure of the wall surface at the inner wall which is not completely smooth and continuous at the point of wall pressure measurement. As can be seen in Figure 5-13 this effect is even more pronounced on the correlation of the wall pressure with the radial velocity component. The correlation of the outer wall pressure and the radial velocity component (Figure 5-14) shows a slightly skewed shape near 


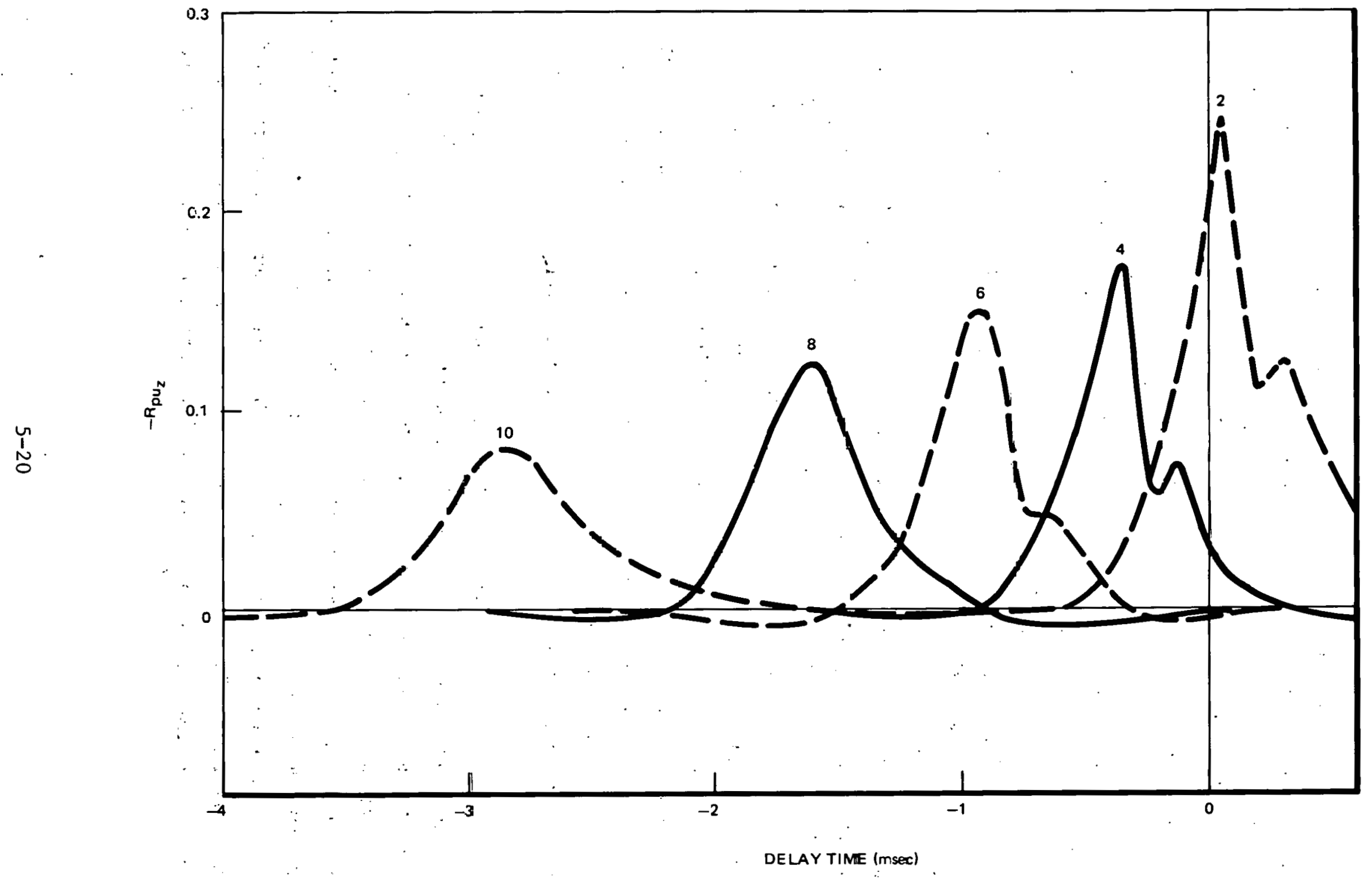

Figure 5-11. Cross-Correlations ketween the Axial Felocity Component and the Inner Wall Pressure as a Function of Lelay Time for Different Axial Separations 


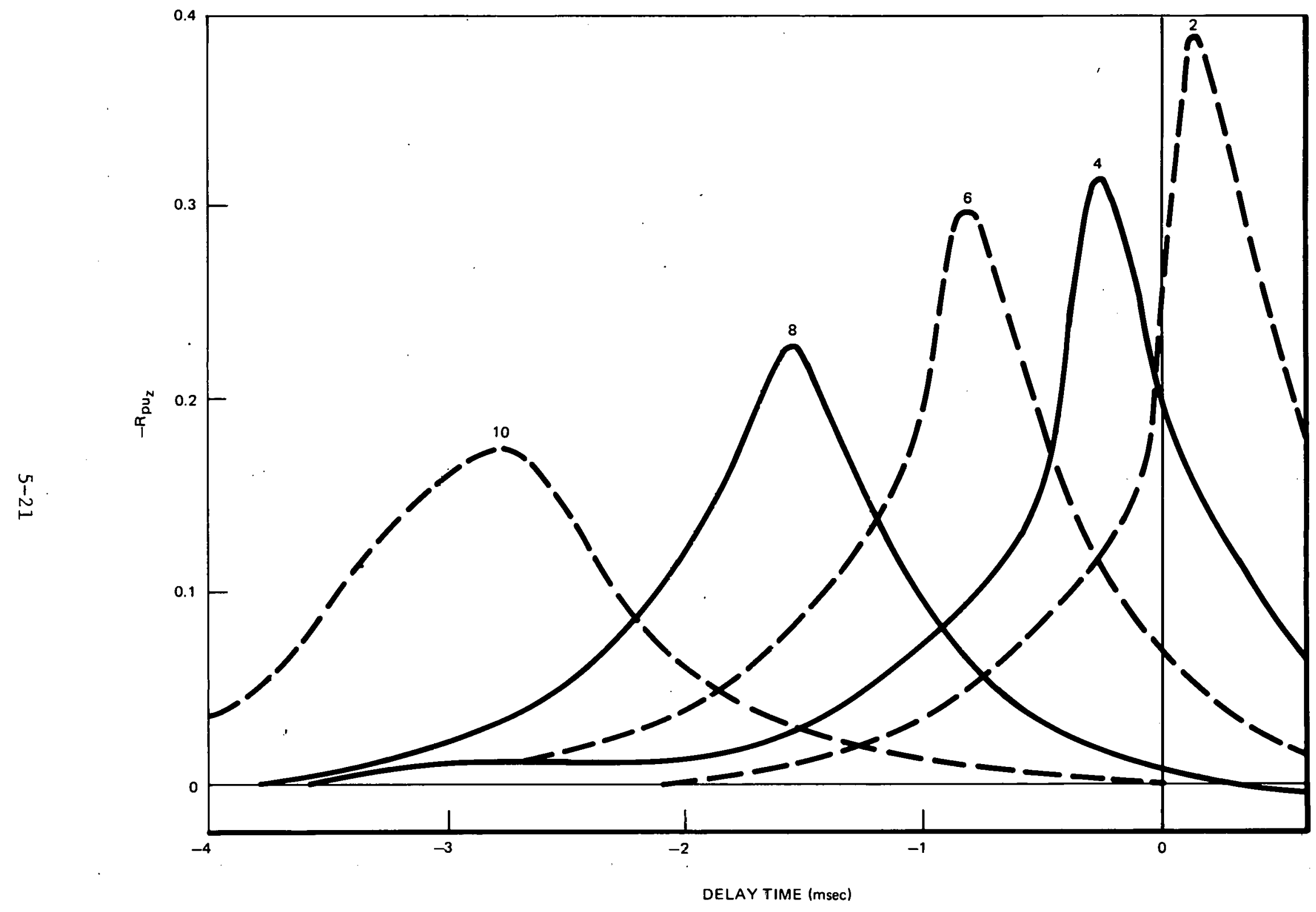

Figure.5-12. Cross-Correlati. ns between the Axial Velocity Component and the Outer Wall Pressure as a Function of Delay Time for Different Axial Separations 


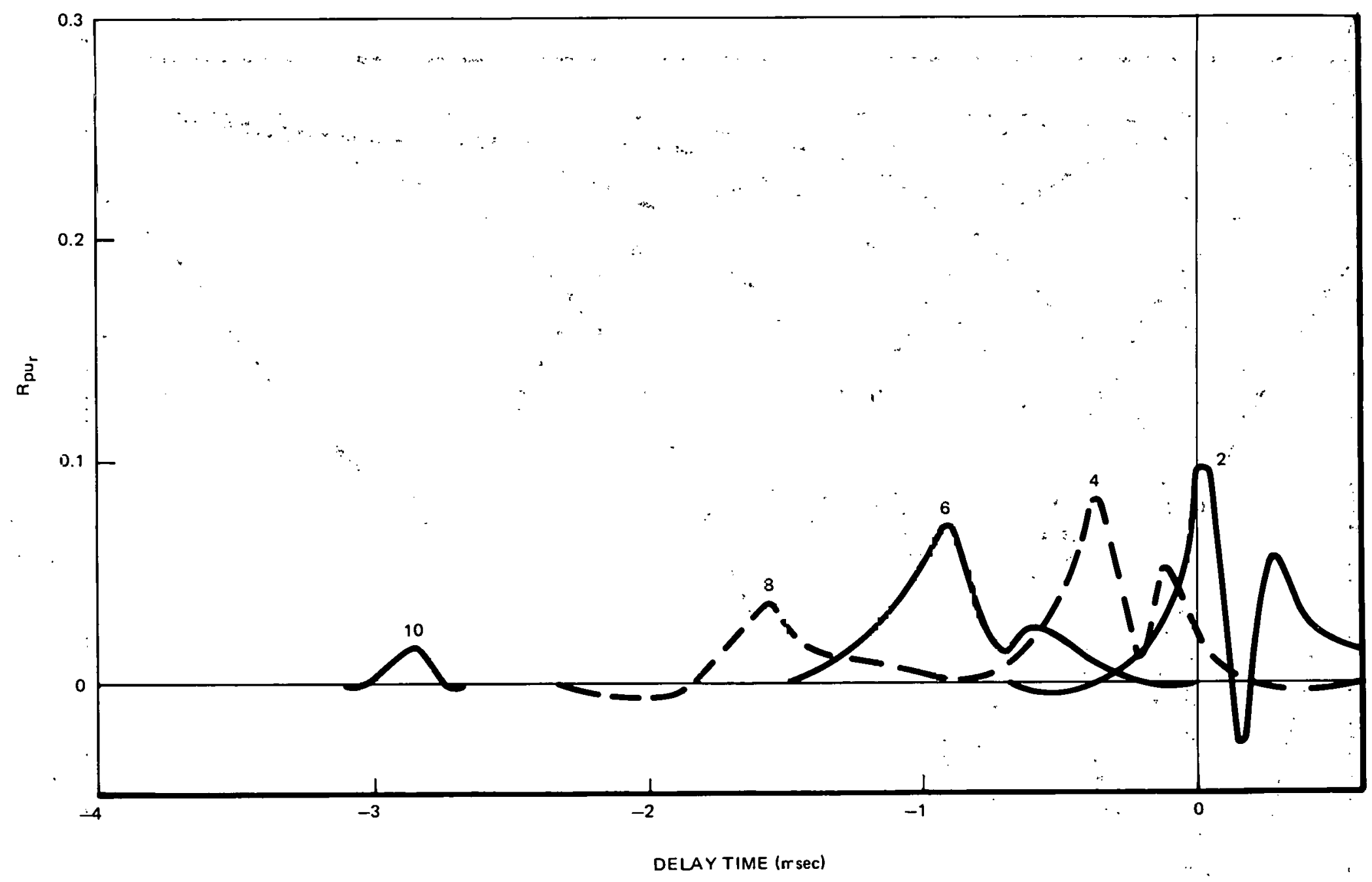

Figure 5-13. Crcss-Correlations between the Radial Velcicity Component and the Inner Wall Prassure as a Function of Delary Time for Different Axial Separations 


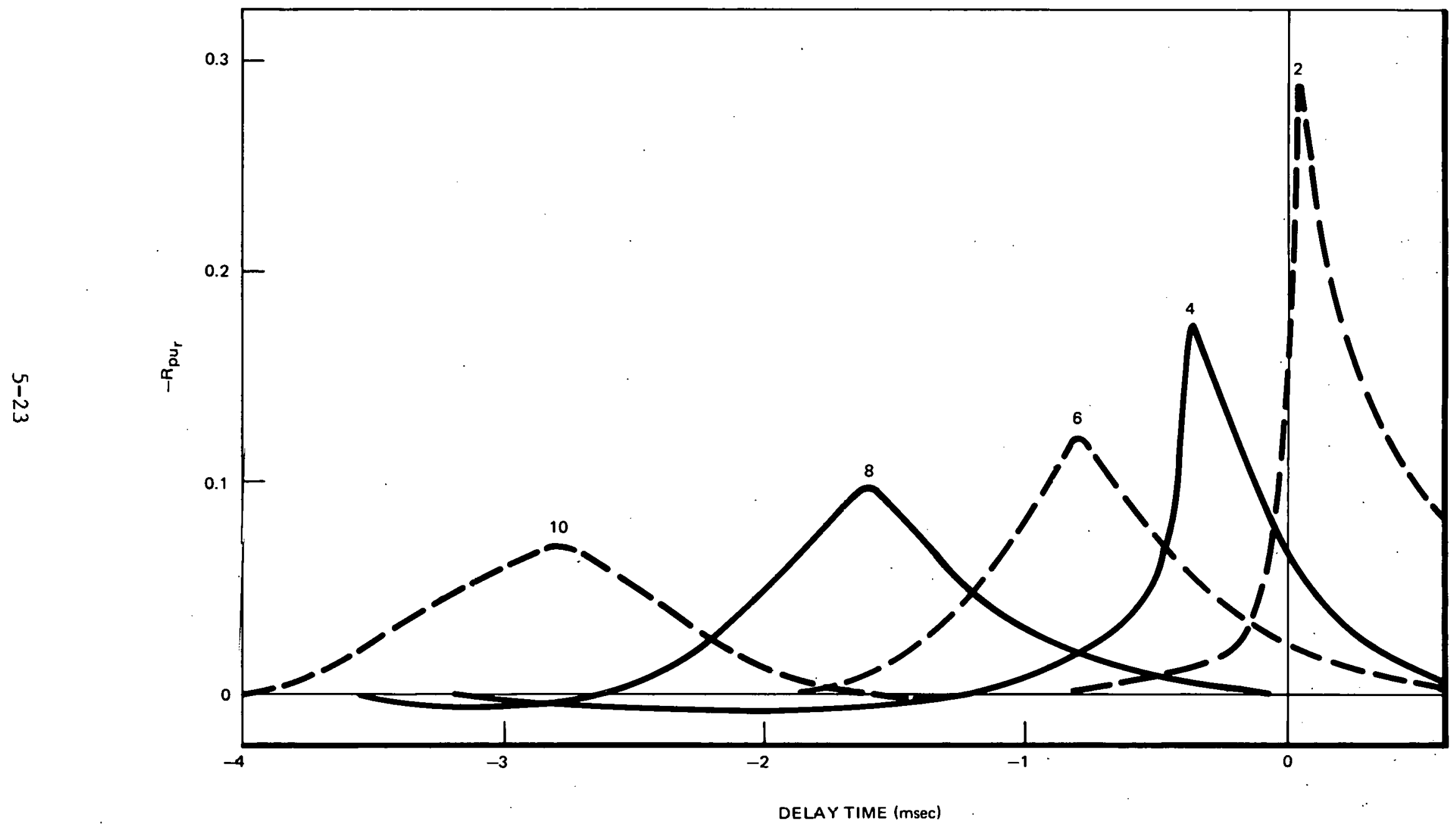

Figure 5-14. Cross-Correlations between the Radial Velocity Component and the Outer Wall Pressure as a Function of Delay Time for Different Axial Separations 
zero separation distances which approaches a more symmetrical shape with increasing axial separation distances. This effect is also seen in Figure 5-12 for the outer wall pressure correlation with the axial velocity component. The magnitude of the correlations is in good agreement with Serafini's data ${ }^{72}$, but considerably less than that of Willmarth and dridge. 77

Figure 5-15 gives the absolute value of the maximum correlations between the wall pressure fluctuations and axial velocity fluctuations as a function of axial separation distance. Figure 5-16 gives the equivalent values for the wall pressure flurtuatione and the radial velocity tlurtuatione. The magnitudes of the correlations show the axial velocity component has a stronger correlation with the wall pressure than the radial velocity compullent. The correlation between the velocity field and the pressure at the outer wall is also larger than it is at the inner wall. This is consistent with the pressure-pressure correlations which were also stronger at the outer wall than at the inner wall for equivalent radial separation distances.

Exponential curve fits to the data of Figures 5-15 and 5-16 were used to calculate integral length scales of the prcsuure-velucily correlations. The resultant length scales are $8.9+/-0.9$ in. for the axial velocity correlation with the outer wall pressure; $6.6+/-0.7$ in. for the axial velocity currelation with the inner wall preusure; $5.2+/=0.51 \bar{n}$. tor the radial velocity correlation with the outer wall pressure; and $4.1+/-0.4$ in. for the radial velocity correlation with the inner wall pressure. The integral length scales from the axial pressure-pressure correlatinns gave $1.2+/-0.4 \mathrm{in}$. for the outer wall and $3.3+/-0.3$ in. for the inner wall. The pressurevelucily correlation appears to exist over longer separation distances than the pressure-pressure correlations based on their relative integral length scales. The actual separations were not great enough to allow any of the axial separation correlations to go to zero.

Figures 5-17 and 5-18 give the convection velocity of the pressure velocity correlations based on axial separation distances and delay time to maximum 


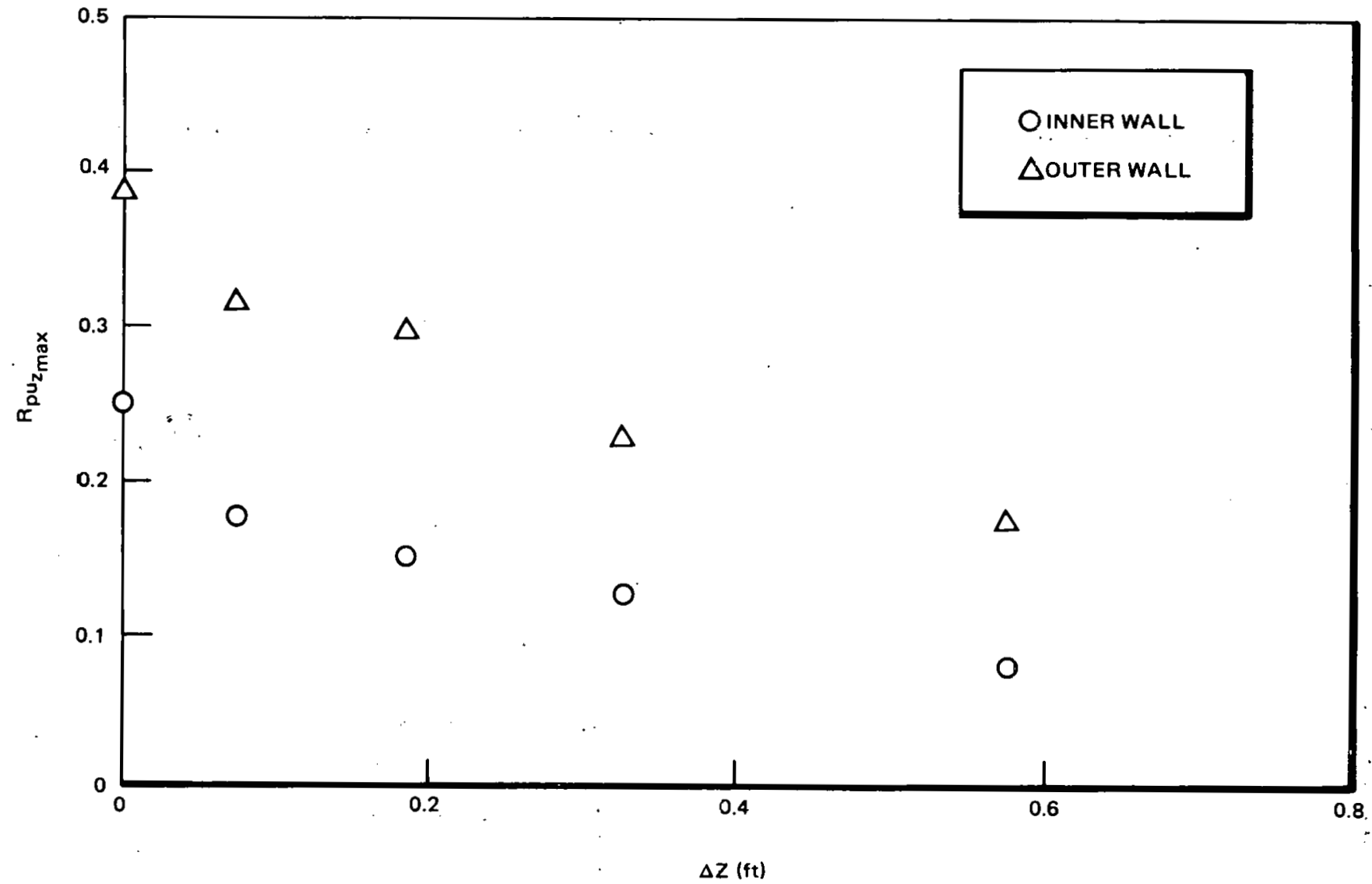

Figure 5-15. The Magnitude of the Maximum Correlation between the Axial Velocity Component and Wall Pressure for Increasing Axial Separation Distances 


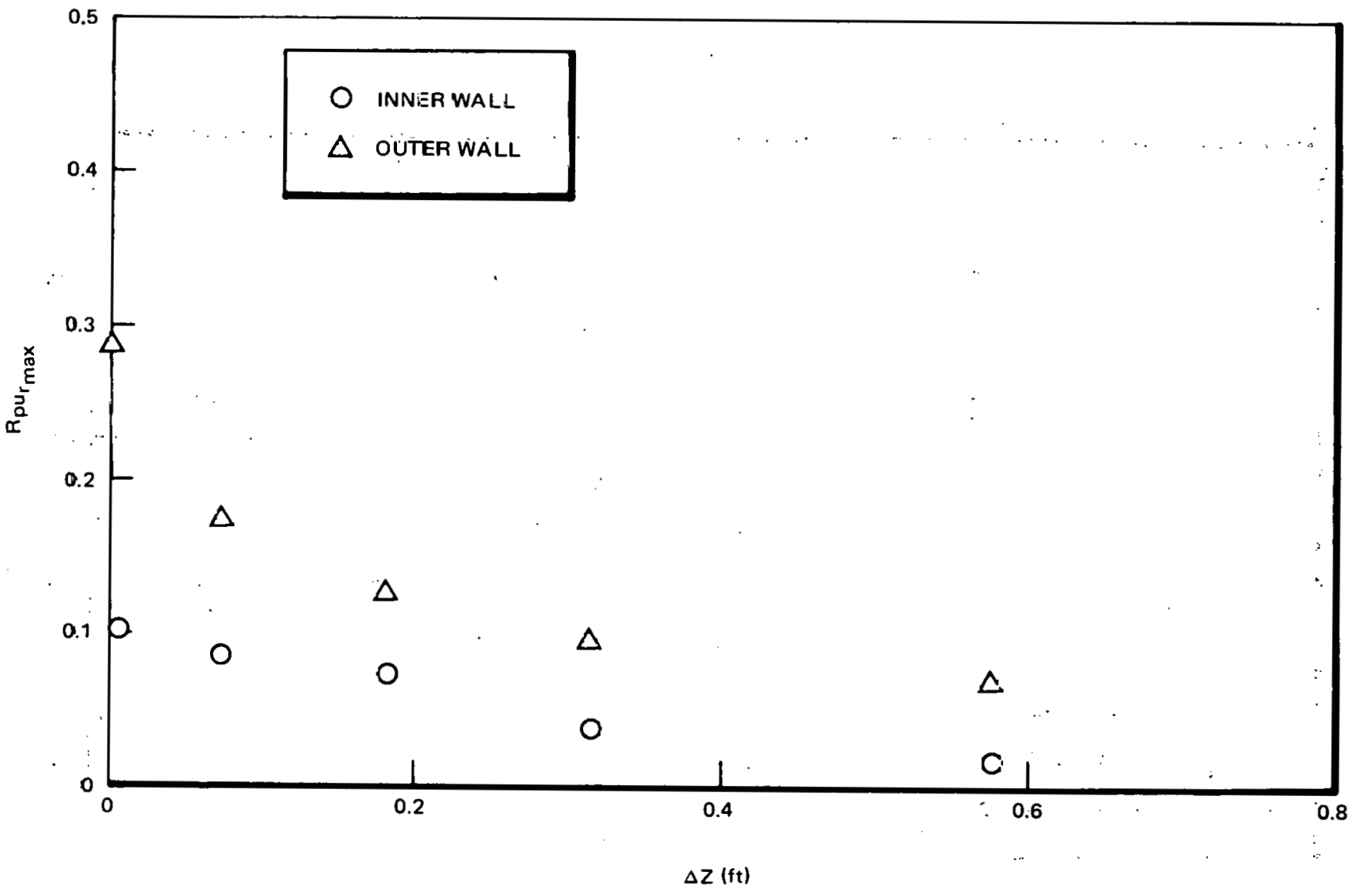

Figure 5-16. The Magnitude of the Maximum Correlation between the Radial Velocity Component and Wall Pressure for In:reasing Axial Separation Distances 


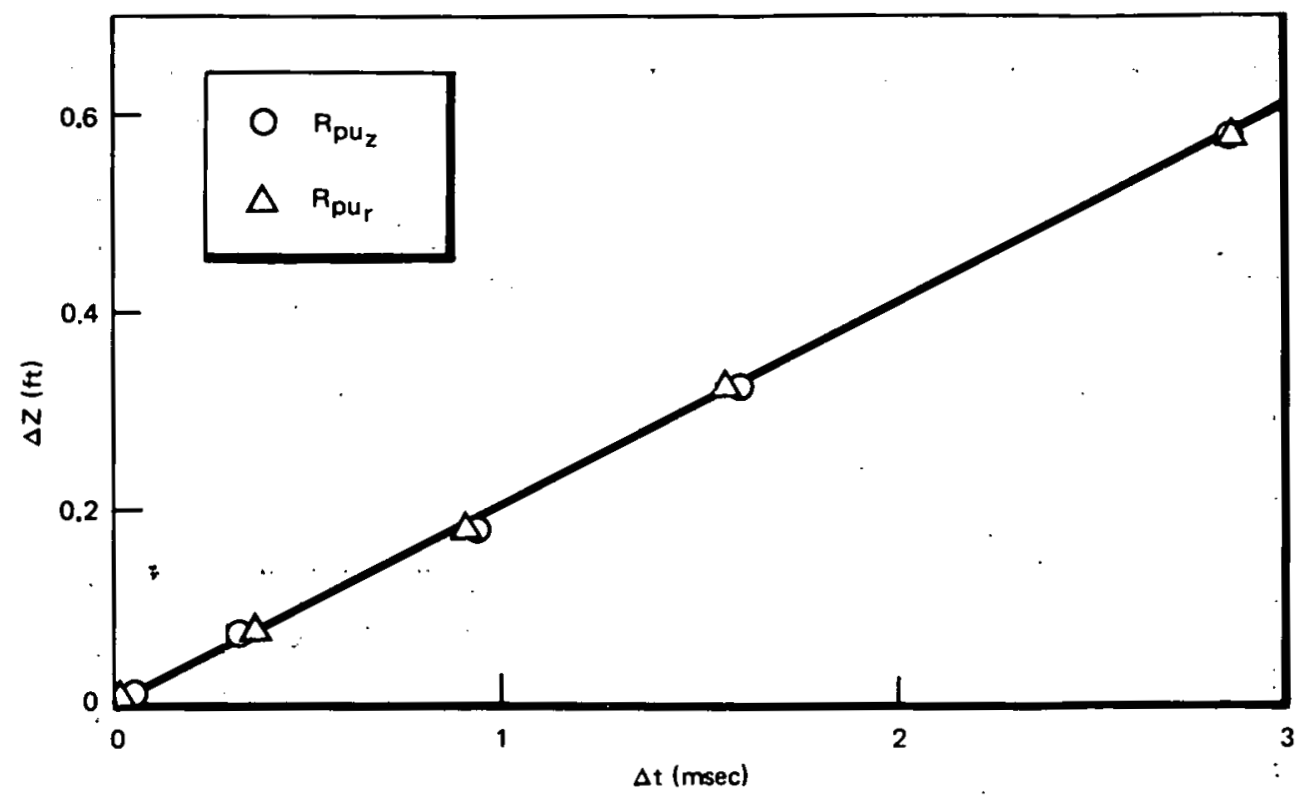

Figure 5-17. Axial Separation Distance Versus Delay Time to Maximum Pressure-Velocity Correlation at the Inner Wall 


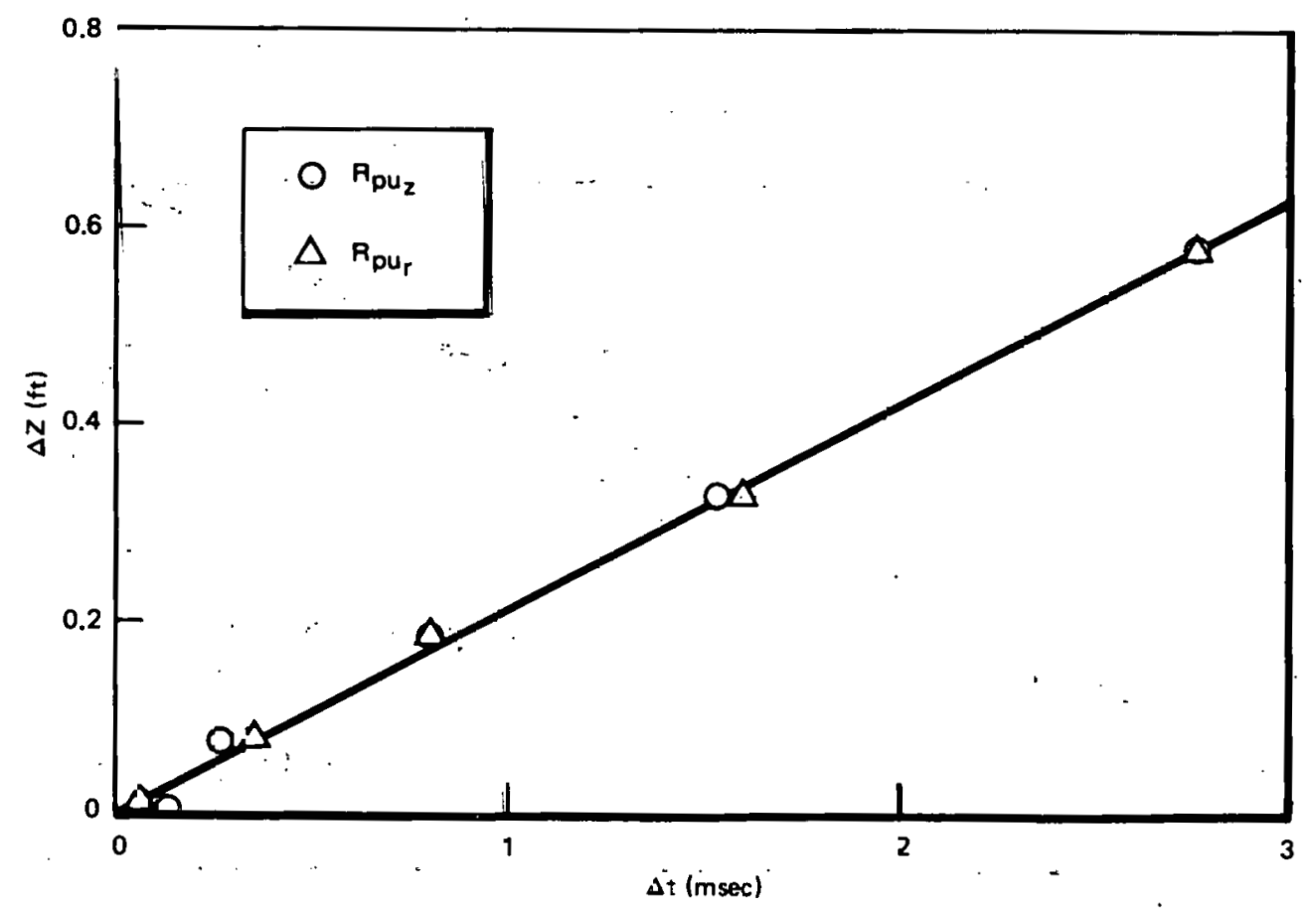

Figure 5-18. Axial Separation Distance versus Delay Time to Maximum Pressure-Velocity Correlation at the Outer Wall 
correlation. The resultant convection velocity for all of the axial separation pressure-velocity correlations is $203 \mathrm{t} /-5 \mathrm{ft} / \mathrm{sec}$. This is in excellent agreement with the pressure-pressure convection velocity which was shown to be $204 \mathrm{t} / \mathrm{-} \mathrm{ft} / \mathrm{sec}$.

The radial correlations between the axial velocity component and the wall pressure are shown in Figures 5-19 and 5-20 for the inner and the outer walls, respectively. A linear extrapolation of the data to the walls gives a correlation magnitude of approximately 0.53 for both walls. Serafini ${ }^{72}$ gives a correlation magnitude of approximately 0.52 for the correlation of the wall pressure with the axial velocity component in a turbulent plane boundary layer. However, the measurements by Willmarth and Wooldridge ${ }^{77}$ give a maximum correlation magnitude of only aboout 0.16 . This difference may be due in part to their rejection of the low frequency wall pressure information.

The integral length scale, defined as the area under the correlation curve, gives a dimensionless $R^{*}$ value of $0.1 .34+/-0.005$ ( 0.111 in.) for the outer wall correlation with the axial velocity component and $0.100+/-0.0005$ (0.083 in.) for the inner wall correlation with the axial velocity component. These values are approximately one half of the integral length scale calculated from the pressure-pressure correlations. This is opposite of the relationship between the axial length scales which gave pressure-velocity integral length scales which were approximately twice that of the pressurepressure integral length scales.

The correlation between the wall pressure and the radial velocity component as a function of radial separation are shown in Figures 5-21 and 5-22 for the inner and outer walls, respectively. The outer wall pressure correlation with the radial velocity component has a similar profile to that of the correlation with the axial velocity component. The magnitude is somewhat lower when extrapolated to the wall (0.40 versus 0.53 ) bul the integral length scale is approximately the same, $0.1 .37+/-0.005$ (0.114 in.). The inner wall pressure correlation with the radial velocity component has a decidedly different profile. This may be due to the different wall pressure 


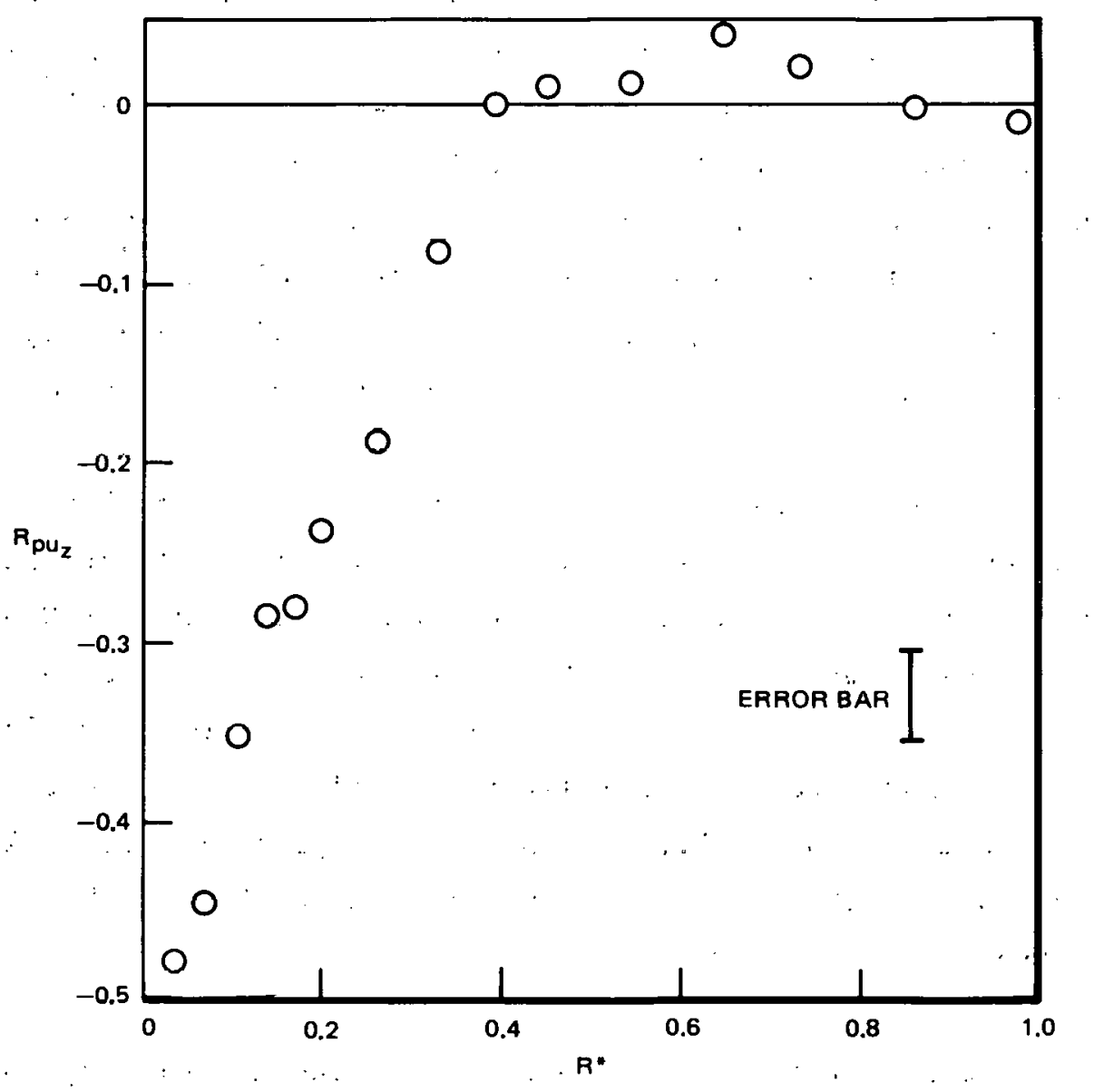

Figure 5-19. Maximum Correlation between the Inner Wal1 Pressure and the Axial Velocity Component as a Function of Radial Separation Distance 


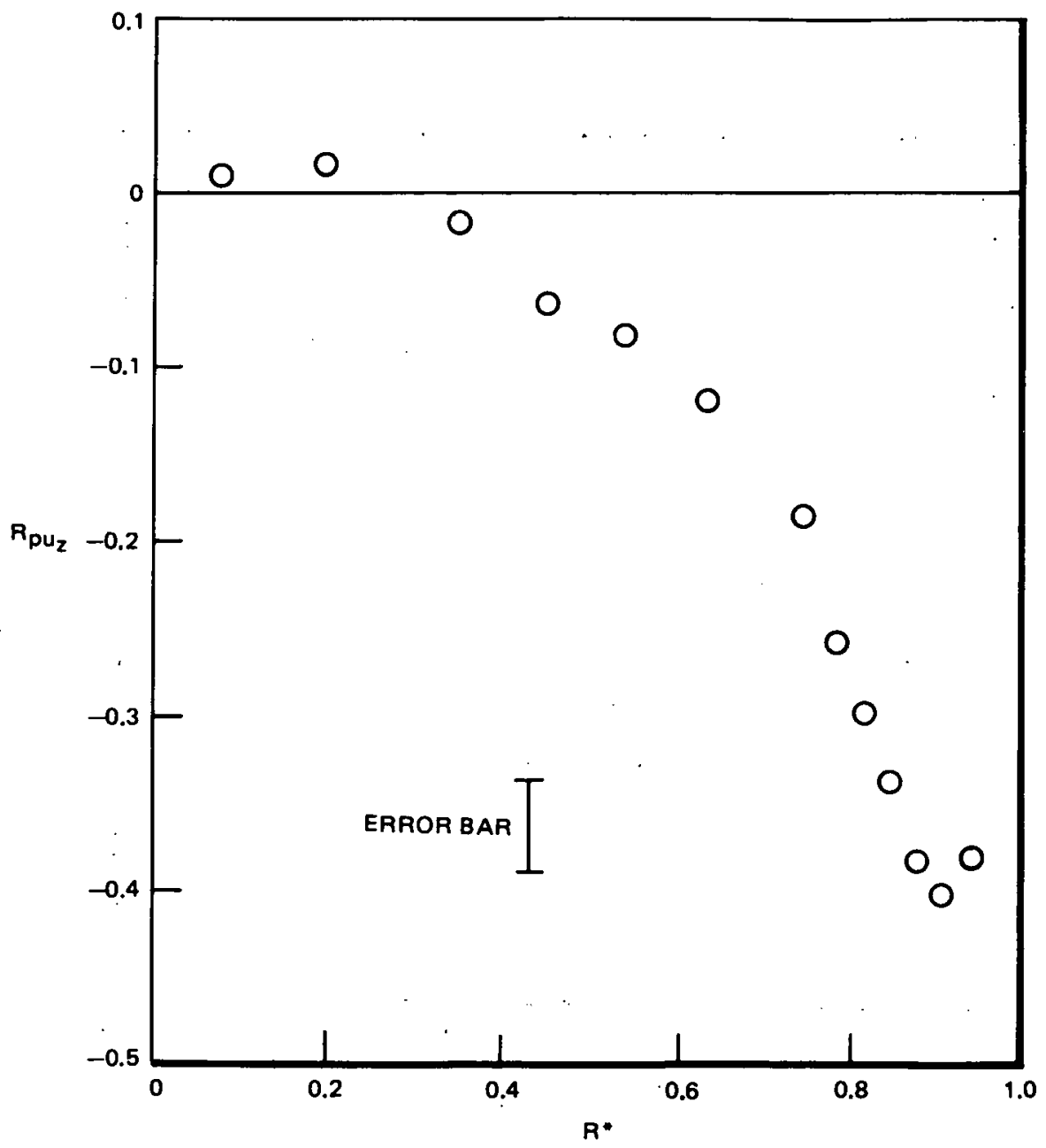

Figure 5-20. Maximum Correlation between the Outer Wall Pressure and the Axial Velocity Component as a Function of Radial Separation Distance 


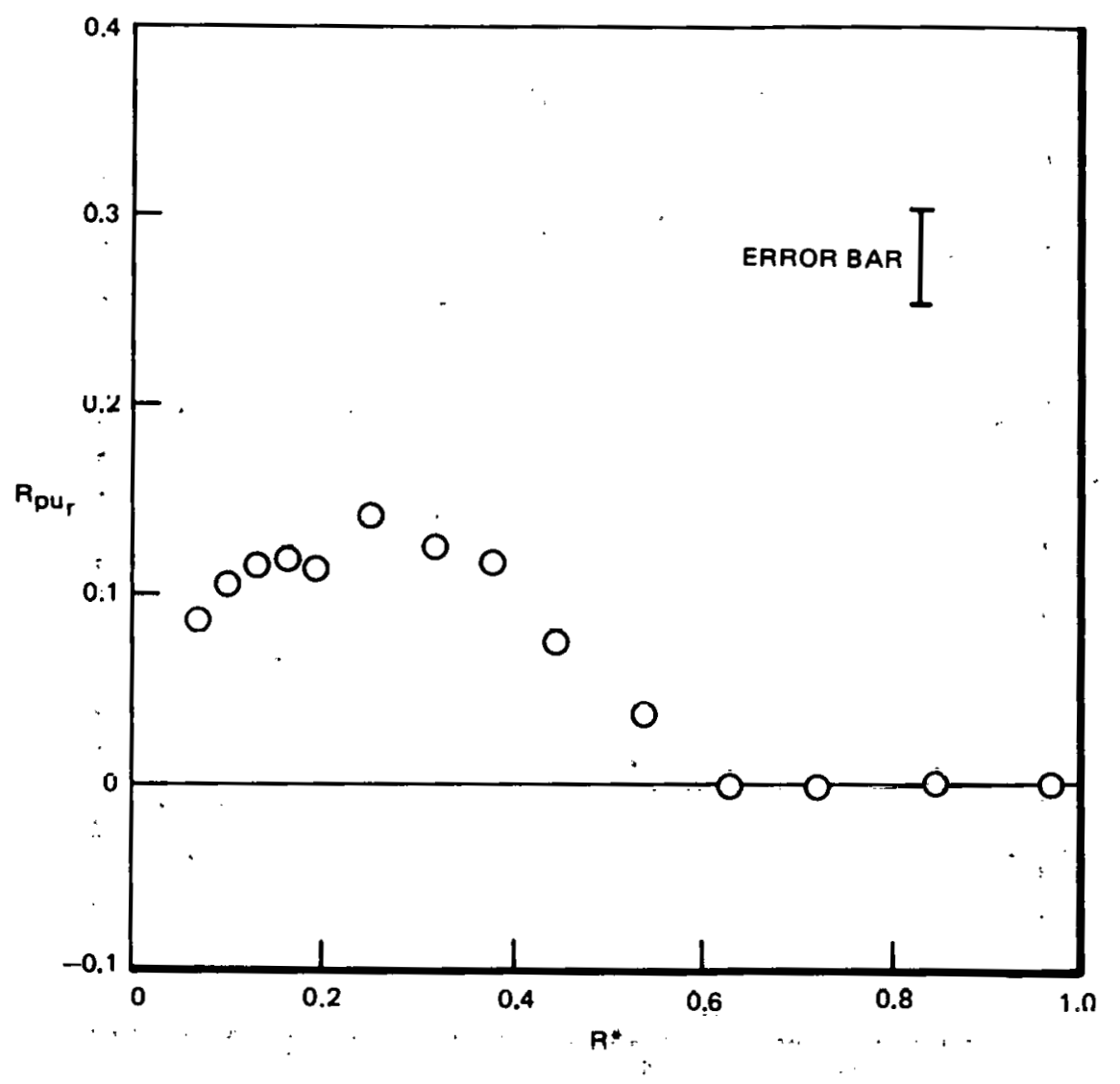

Figure 5-21. Maximum Correlation between the Inner Wall Pressure. , and the Radial Velocity Component as a Function of Radial Separation Distance. 


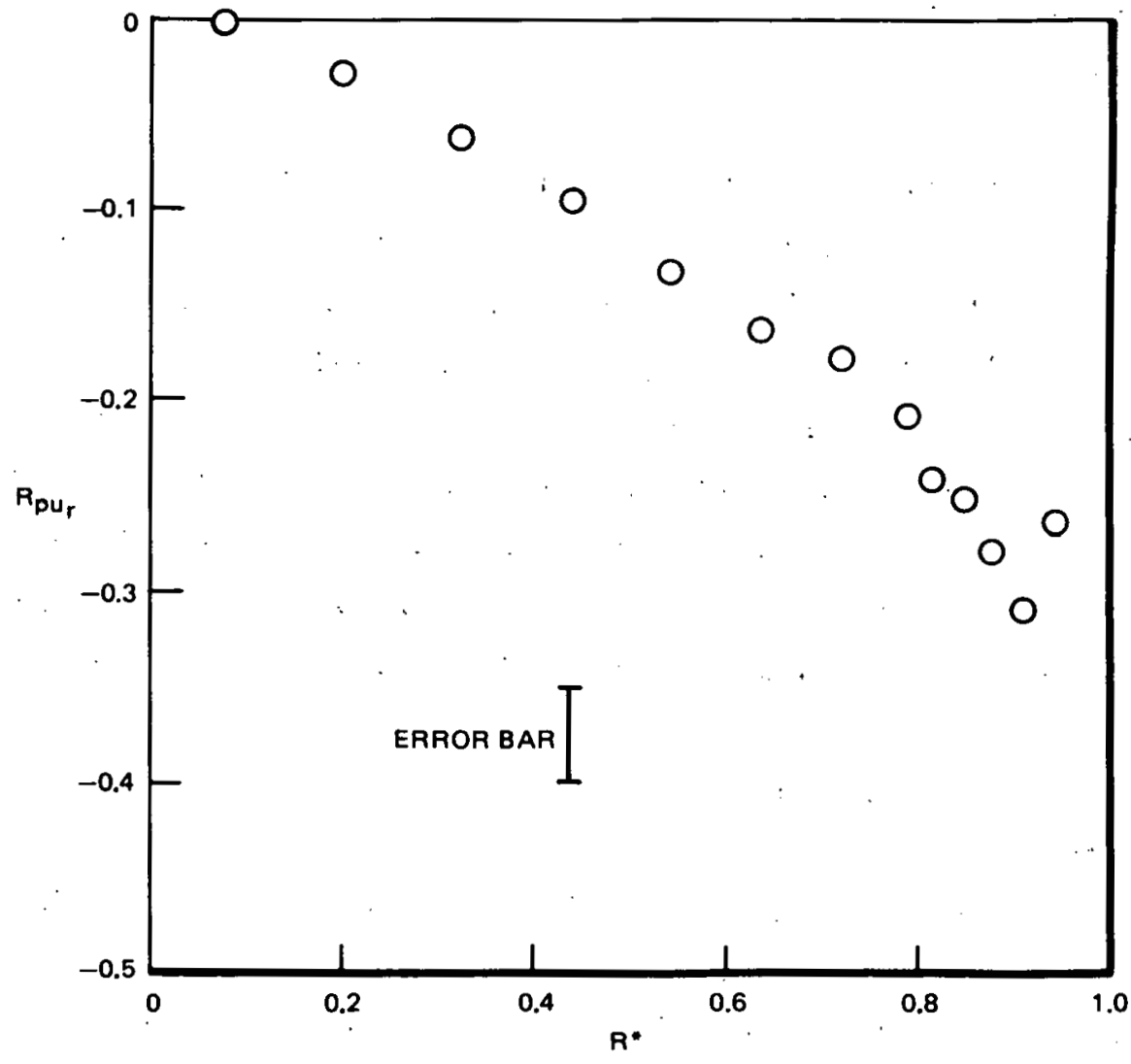

Figure 5-22. Maximum Correlation between the Outer Wall Pressure and the Radial Velocity Component as a Function of Radial Separation Distance 
transducer mountings on the two walls. The inner wall at the pressure sensing location is not rigid but rather open to absorbent sponge like foam. This foam may absorb some of the dynamic pressure created on the surface by normal velocity fluctuations. However, there was no evidence of this effect in the wall pressure intensity or spectral measurements. For $R^{*}>0.25$ the correlation follows the general profile of the other pressure-velocity correlations, but then levels off for separation distances of $R^{*}<0.25$. The integral length scale calculated from Figure 5-21 gives $0.056+/-0.005$ (0.046 in.), which is considerably less than that of the axial velocity correlation with the inner wall pressure.

The pressure-velocity correlations as a function of azimuthal separation are shown in Figures 5-23,5-24, 5-25 and 5-26. Al1 of these correlations show a very similal proftle, changing only in magnitule and pulartey. All ot these correlations dip rapidly through zero, peak and then return to zero. This correlation profile would be the expected result nf a rotational flow in the $\hat{z}-\hat{\phi}$ or $\hat{r}-\hat{\phi}$ plane. This would suggest that some sort of vortex or rotational flow exists in the core region of the flow field. Willmarth ${ }^{86}$ has found evidence of streamwise vorticity in boundary layer flow. "This streamwise vorticity would imply that the vorticity could be in the $\hat{z}-\hat{\phi}$ plane.

The integral length scales are very small for these correlations as the magnitudes of the correlations are relatively low. Two interesting obocrvations are made on these correlations. First the peak values of the correlations after the zero crossing occur at. separation distances. which are of equal linear distance for the two walls. For the axial velocity component and inner wall pressure this value is $0.24+/-0.02 \mathrm{in}$. and for the outer wall it is $0.31+/-0.02$ in. Similarly for the radial velocity component the values are $0.14+/-0.02$ in. at the inner wall and $0.17+/-0.02$ in. at the outer wall.

Secondly, the same observation can be made for the zero crossings themselves. The axial velocity component correlation with the inner wall occurs at a linear separation distance of $0.14+/-0.01 \mathrm{in}$. and that of the outer wall at $0.14+/-0.01$ in. Similarly the correlations with the radial velocity component give values of $0.11+/-0.01$ in. for the inner wall and $0.12+/$ - $0.01 \mathrm{in.}$ for the outer wall. These results are in contrast to the integral 


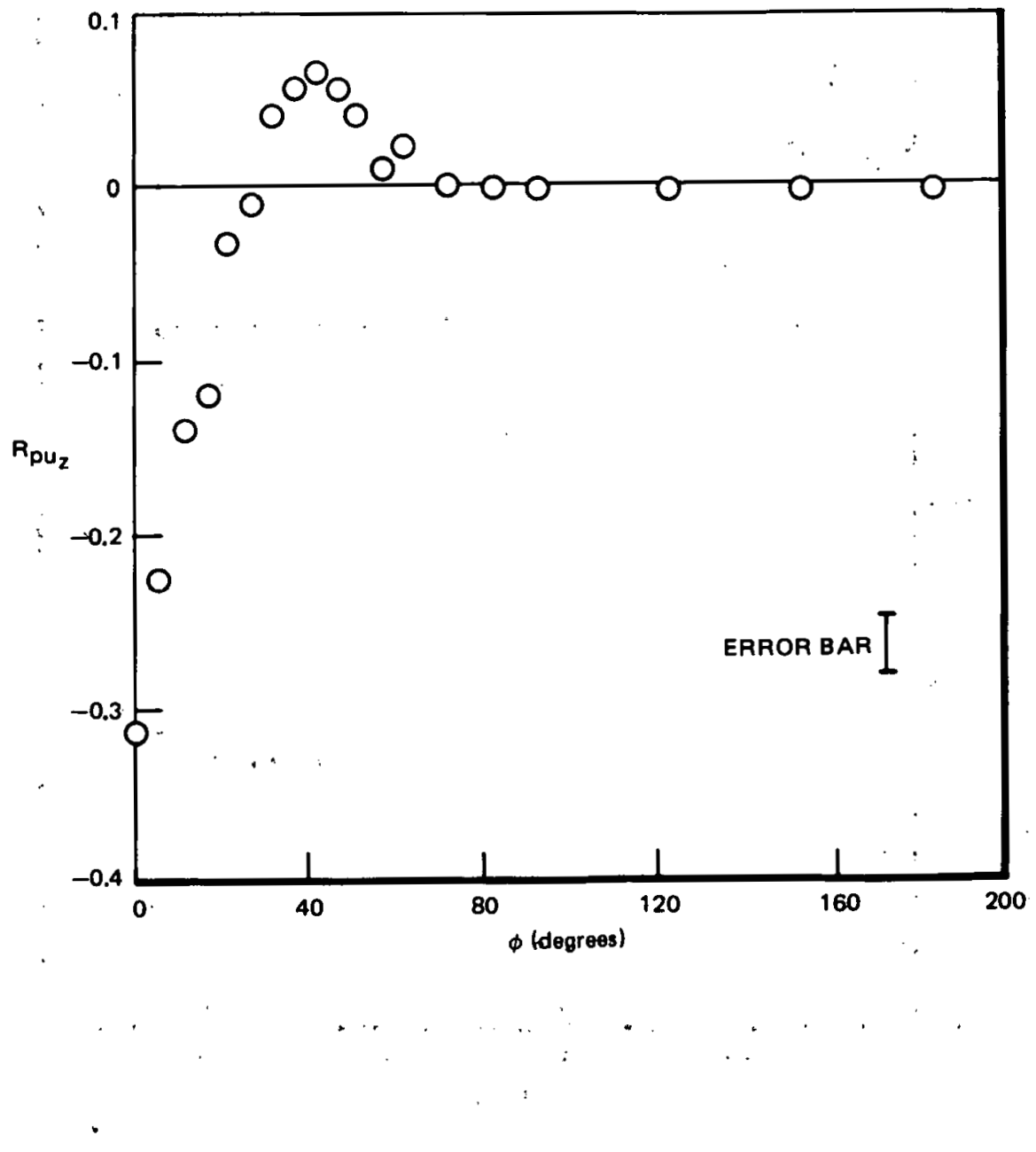

o

Figure 5-23. Maximum Correlation between the Inner Wall Pressure and the Axial Velocity Component as, a Function of Azimuthal Séparation Arglé 


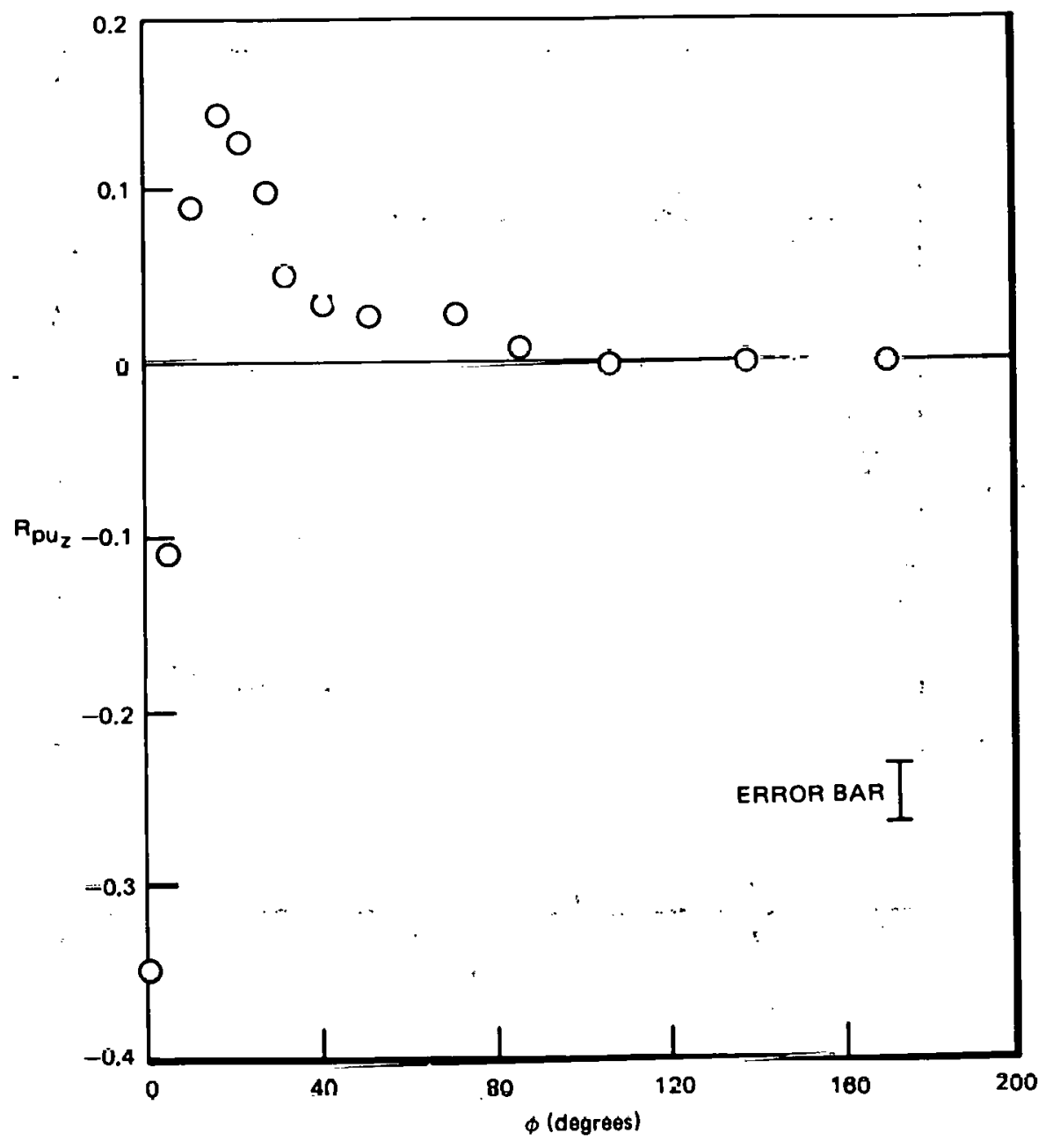

0

Figure 5-24. Maximum Correlation between the Outer Wall Pressure and the Axial Velocity Component as a Function of Azimuthal Separation Angle 


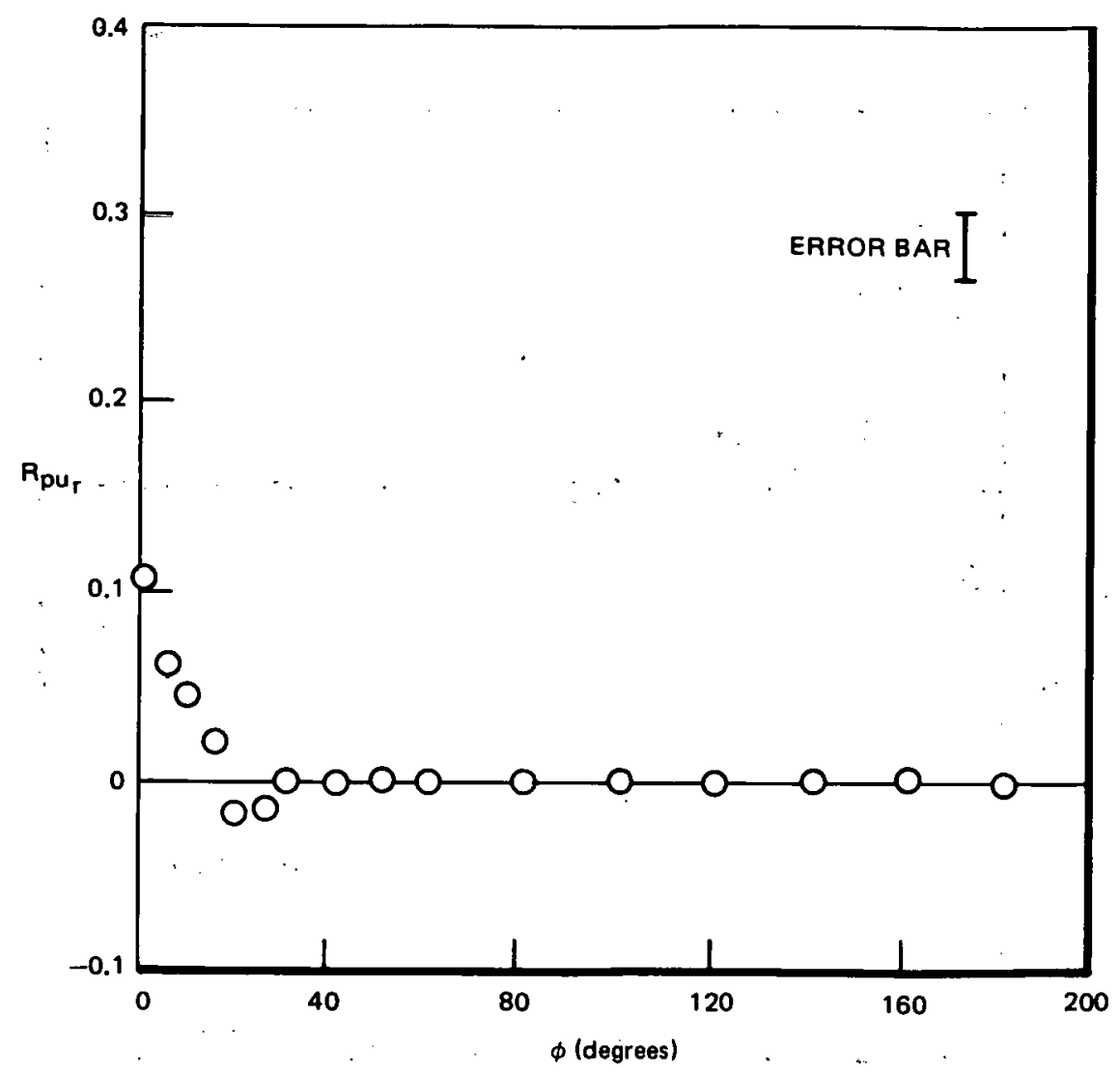

Figure 5-25. Maximum Correlation between the Inner Wall Pressure and the Radial Velonity. Component as a Function of Azimuthal Separation Angle 


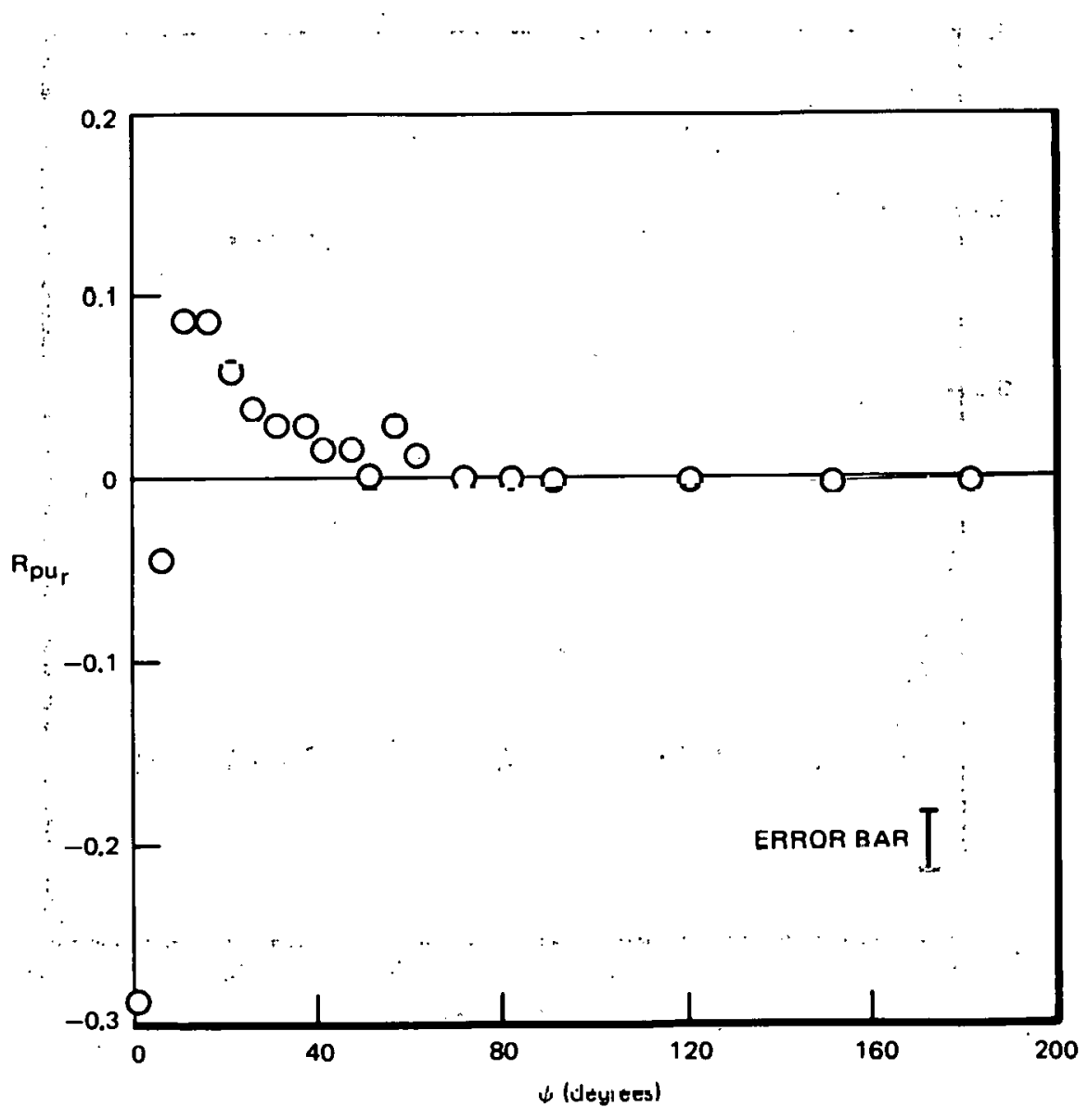

Figure 5-26. Maximum Correlation between the Outer Wall Pressure and the Radial Velocity Component as a Function of Azimuthal Separation Angle 
length scales for the pressure-pressure correlations which were about $25 \%$ larger at the outer wall.

The pressure-velocity correlations with radial separation showed an increasing time lag to maximum correlation for the axial velocity component, but no change for the radial velocity component. This is shown in Figure 5-27. This time lag for the axial velocity component was also noted by Serafini ${ }^{72}$, but no explanation was offered. Willmarth and Wooldridge's data ${ }^{77}$ appear to be similar in this respect, but no detailed information is presented.

It is somewhat surprising to find a time lag existing only for the axial velocity component correlation. This time lag is shown to be nearly linear with separation distance (Figure 5-27). The "Convection" velocity based on delay time to maximum correlation and separation distance gives a value of approximately $85 \mathrm{t} /-15 \mathrm{ft} / \mathrm{sec}$ at the outer wall and $110 \mathrm{t} /-15 \mathrm{ft} / \mathrm{sec}$ at the inner wall. This appears to be consistent with velocity-velocity correlations in a turbulent boundary layer measured by Favre, et al. ${ }^{138}$ Their data shows an increasing "convection" velocity for increased distances frum the wall. This appears to imply that this value is a function of the local mean velocity which also increases with increasing separation distance from the wall. Thus the inner wall value, which is at a higher local mean velocity, should have a time delay value which is less than that of the outer wall, which is at the same separation distance but at a lower local mean velocity.

It would appear that the fluctuations in the axial velocity component must be transmitted to the wall through some drag effect of one fluid element on another. A fluctuation in the axial velocity component in the core regluil of the flow must transfer its effect to the wall by accelerating neighboring fluid elements until this fluctuation is transmitted to the wall. It can then be speculatively hypothesized that this axial velocity fluctuation causes a fluctuation in the wall pressure by a Bernouli effect, i.e., increasing velocity fluctuations cause decreased wall pressure and vice versa. This change in the wall pressure can then drive the fluid in the radial direction, either away from or toward the wall, depending on the normal forces acting on 


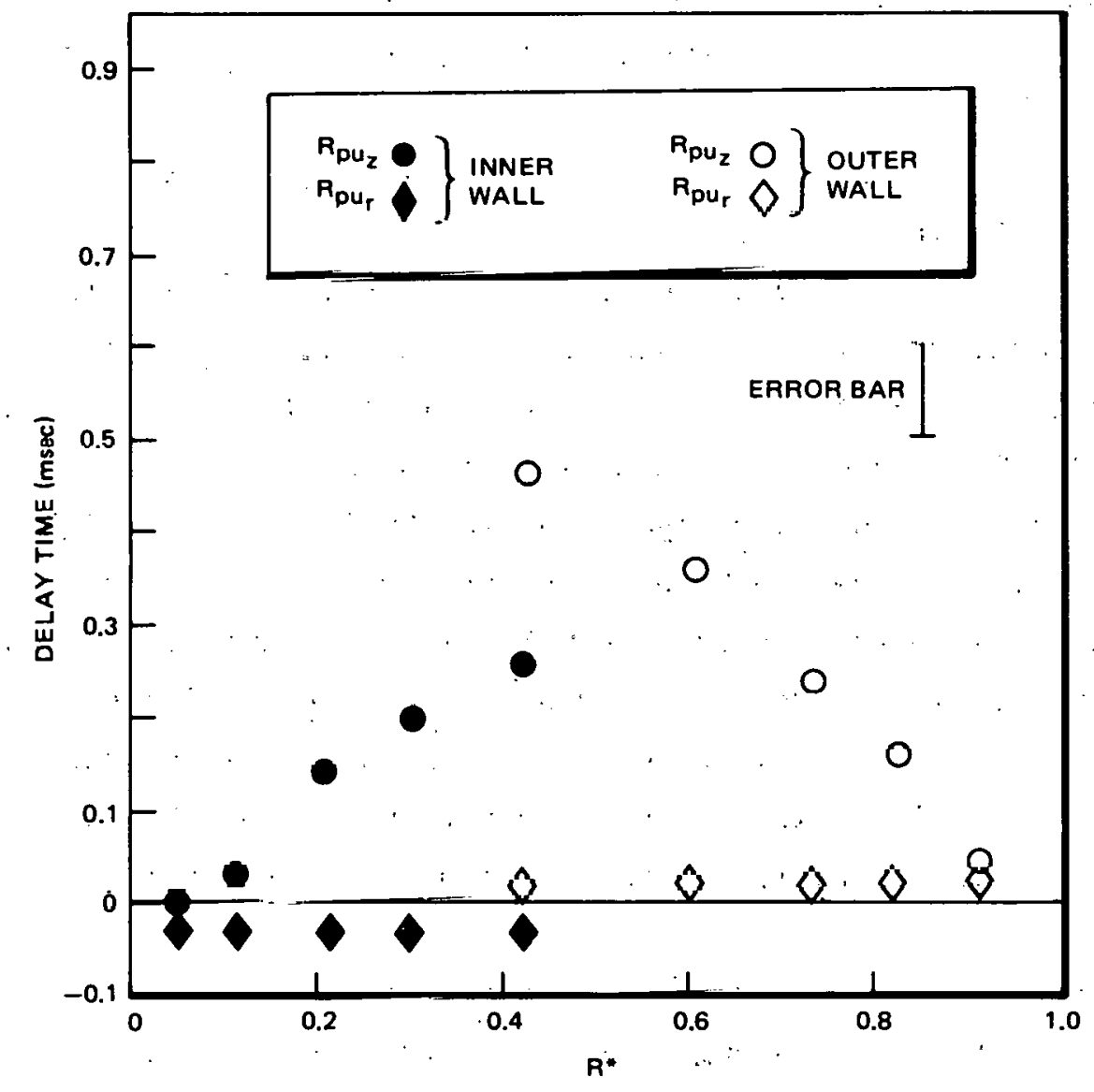

Figure 5-27. Delay Time to Maximum Pressure-Velocity Correlation as a Function of Radial Position 
the fluid.. From the data of Figure 5-27 this effect must happen very rapidly as no discernible time delay could be found between the maximum correlation of the wall pressure and radial velocity component for different radial separations. If this effect propagated at or near the sonic velocity the resultant "convection" velocity could not be measured with the time delays used in this investigation. The resultant time delays to maximum correlation of Figure 5-27 are also consistent with the hypothesis that the axial velocity component drives the pressure which in turn drives the radial velocity component and presumably the azimuthal component as well. This pressure-velocity interaction is not presumed to be the complete description of the mechanism involved, but is a possible scenario of an interaction which is consistent with the data.

It is possible to generate a velocity field based on the pressure-velocity correlations, if it is assumed that the velocity in the ith direction at time $t+\tau$ is related to the pressure at time $t$, i.e.,

$$
u_{i}(t+\tau)=g(p(t))
$$

This approach at generating the velocity field from correlations is taken from Adrian ${ }^{139}$ who applied it to velocity-velocity correlations.

The simplest functional relationship between the pressure and velocity would be a linear function, i.e.,

$$
u_{i}(t+\tau)=g_{i}(\tau) p(t) \quad
$$

The optimum relationship for $g_{i}(\tau)$ would be that which gives a minimum mean square error. The mean square error is defined as

$$
e(\tau)=\left\langle\left[u_{i}(t+\tau)-g_{i}(\tau) p(t)\right]^{2}\right\rangle
$$


where the braces denote a long time average. The function, $g_{i}(\tau)$, which produces the minimum error can be found by taking the derivative of Equation (5-9) with respect to $g_{i}(\tau)$ and setting it equal to zero, i.e..

$$
\frac{\ddot{d e}(\tau)}{d g_{i}(\tau)}=0=\left\langle\left[2 u_{i}(t+\tau)-g_{i}(\tau) p(t)\right] p(t)\right\rangle
$$

Solving Equation $(5-10)$ for $g_{i}(\tau)$ yields

$$
g_{i}(\tau)=\frac{u_{i}(t+\tau) p(t)}{\left\langle p^{2}(t)\right\rangle}
$$

The correlation between the velocity component in the ith direction and the pressure is defined as

$$
\mathrm{R}_{u_{i} p}=\frac{\left\langle u_{i}(t+\tau) p(t)\right\rangle}{\left\langle u_{i}^{2}(t)\right\rangle^{1 / 2}\left\langle p^{2}(t)\right\rangle^{1 / 2}}
$$

Subst1tuting this into Equation (5-i1) yields

$$
g_{i}(\tau)=\frac{k_{u_{i} p}(\tau)\left\langle u_{i}^{2}(t)\right\rangle^{1 / 2}}{\left[p^{2}(t)\right]^{1 / 2}}
$$

Thus, the velocity component in the ith direction at time $t+\tau$ hased on a linear estimation glves

$$
u_{i}(t+\tau)=\frac{R_{u_{i} p}(t)\left\langle u_{i}{ }^{2}(\tau)\right\rangle^{i / 2} p(t)}{\left\langle p^{2}(t)\right\rangle^{1 / 2}:} .
$$

By conditionally averaging on the pressure signal, $p(t)$, it is possible to estimate the velocity. field component in the ith direction which is associated 
with this pressure fluctuation. Arbitrarily selecting a conditional average on $p(t)$ equal to one standard deviation, $\left\langle p^{2}(t)\right\rangle^{1 / 2}$, yields .......

$$
\left.u_{i}\left[(t+\tau) \mid\left\langle p^{2}(t)\right\rangle^{1 / 2}\right]=\hat{u}_{i}(t+\tau)=R_{u_{i} p}(\tau)\left\langle u_{i}^{2}(t)\right\rangle\right\rangle^{i / 2} \text {. }
$$

For the two velocity components measured in this investigation and the identity $R_{u_{i} p}(\tau)=R_{p_{i}}(-\tau)$, estimated velocity components in the $\hat{z}: \hat{r} e \cdot \hat{r}$ direction are given as

$$
\hat{u}_{z}(t+\tau)=R_{p u_{z}}(-\tau)\left\langle u_{z}^{2}(t)\right\rangle{ }^{1 / 2}
$$

and

$$
\hat{u}_{r}(t+\tau)=R_{p_{r}}(-\tau)\left\langle u_{r}^{2}(t)\right\rangle 1 / 2
$$

respectively.

By using the Taylor hypothesis of a frozen field being swept by the point of measurement at the local mean velocity, $=z / U\left(R^{*}\right)$, a velocițy. vector field in the $r-z$ plane can be obtained from time correlations of the wall pressure and velocity field with radial separation correlations only. Ideally one would like to obtain the correlations of the wall pressure and velocity field at all points in the $r-7, p l a n e$ and use Equations (5-16) and (5-17). However, this is very time consuming and the frozen field assumption will allow the mapping of the estimated velocity field from a small number of correlations.

The velocity field obtained from the linear.estimation theory is shown in Figure 5-28." The velocity vectors for $R^{*}>0.35$ are based on the outer wall correlations and those for $R^{*}<0.35$ are for the inner wall correlations. The correlation values for separation distances beyond the centerline are too small to accurately predict the velocity vector. 


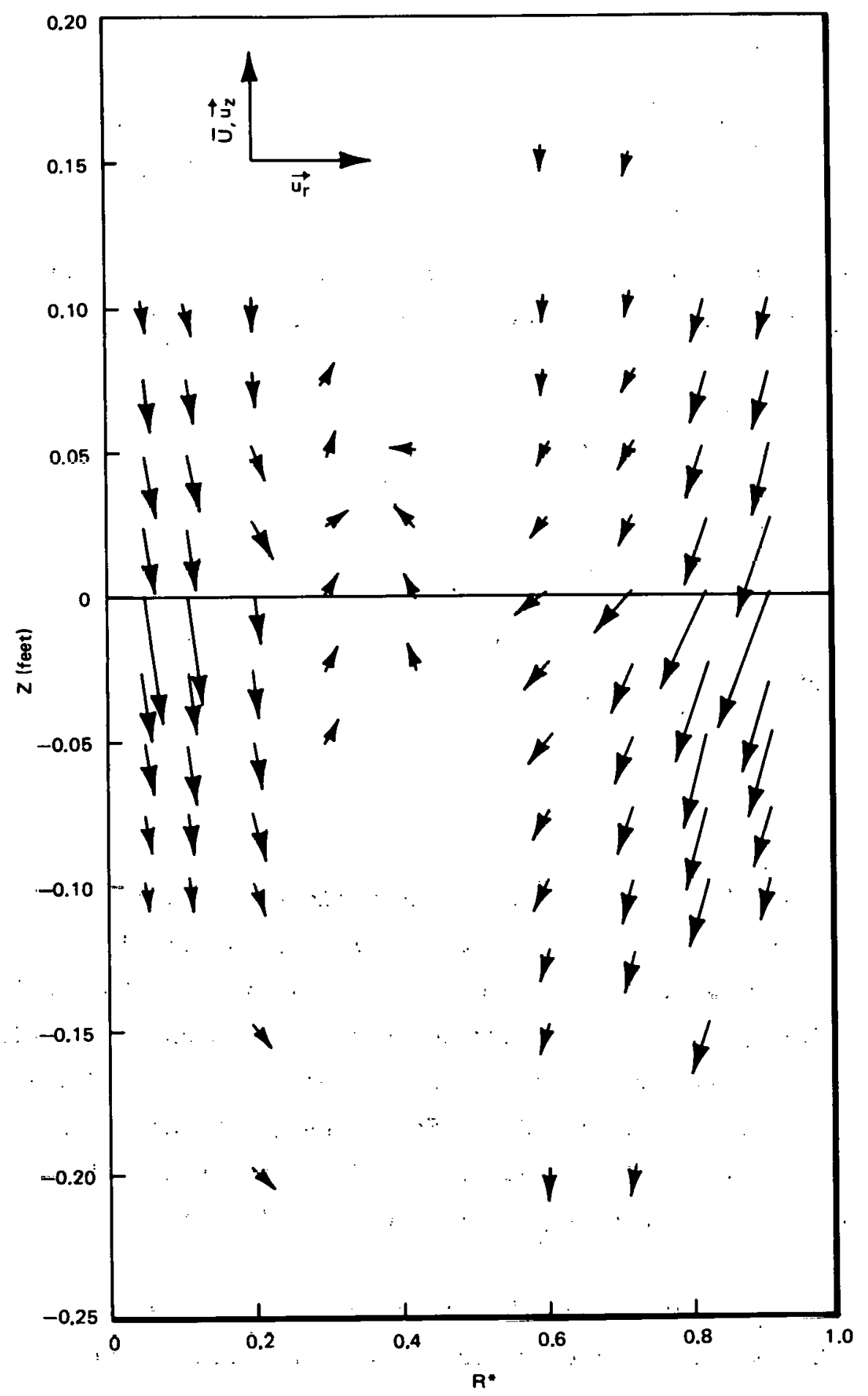

Figure 5-28. Velocity Field as Estimated from the Pressure-Velocity Correlations Using Linear Estimation Theory 
The velocity field of Figure 5-28 is based on $p(t)$ being positive. If $p(t)$ were taken to be negative, all of the velocity vectors would be rotated 180 degrees with the magnitude of each remaining the same. With either selection of $\mathrm{p}(\mathrm{t})$ it is found that the velocity vectors are consistent with the shear". stress which was s̈hown in Figure 4-4. Multiplying the two velocity components together at any point away from the point of zero shear stress will give the correct sign to the shear stress based on the defined geometry orientation: Near the point of zero shear the velocity vectors become small in magnitude... and their direction is not well defined. This can be interpreted as implying that the shear stress in that region is approximately zero as predicted by the linear estimation theory. Conversely the magnitudes of the velocity vectors of Figure 5-28 increase as the point of measurement gets closer to the wall. This velocity field infers a strong relationship between the shear stress in the fluid and the ultimate wall pressure. This is not too surprising in as much as the wall pressure intensity level as a function of mean velocity collapses to a constant when normalized by the shear stress.

The predicted velocity field is only two-dimensional and can not give a complete picture as to the actual flow velocities in the channel. It would appear from Figure 5-28 that there may be two large eddies which are essentially rolling along the walls. This is not explicit as the structure near the point of maximum velocity is not well defined and the error in this region is at least as large as the vectors themselves. Thus the vectors in this region may be in error in direction as well as magnitude. The eddy structure rolling along the walls would be expected to give time correlations similar to those measured by Willmarth and Wooldridge ${ }^{77}$ which is not the case as those measured in this investigation are quite different. Thus it is felt that the velocity field shown is most strongly related to the mean shear stress which in turn interacts with the turbulence to generate the largest contribution to the wall pressure fluctuations.

The small angles that the velocity vectors make with the wall surface are indicative of the large elongation of the velocity-velocity, pressurevelocity and pressure-pressure correlations which have been measured in 
confined:flows and boundary layers. The angle tends to increase as the separation distance from the wall increases. This implies that more of the contribution to; or from, the wall pressure at these points is due to the radial velocity component than that due to the axial velocity component. Thus, if we assume an axial velocịty fluctuation drives the.wall pressure by a Bernoulli effect and that this pressure fluctuation will. in turn drive the radial velocity component, (possibly even causing a bursting effect, on the wall surface) we would expect the velocity vectors to be at larger angles as the separation distance from the wall is increased. In other words the effeçt of an axial velocity fluctuation on the wall will tend to damp out more as the separation distance increases while the "bursting", effect on the wall will drive the normal velocity component rapidly away from the wall, which can be felt at larger distances in the radial direction.

The pressure-velocity correlation measurements show a strong relationship between the wall pressure fluctuations and the fluctuating velocity field. The correlations are consistent with that expected from a pressure-velocity interaction of the axial velocity component driving: the pressure and the. resultant pressure.fluctuation driving the radial, and:presumably the azi- . muthal, velocity components. This interaction appears to be the dominating mechanism as observed from the long time average, wide frequency band, correlations. An investigation using conditional-averaging or narrow. frequency bands may be useful.:in breaking down the interaction mechanisms that may be occurring in different regions or frequencies of the turbulence. 
6.: SUMMARY, CONCLUSIONS AND RECOMMENDATIONS. FOR FUTURE RESEARCH

The research presented in this report was based upon an -investigation of the relationship between the flow field turbulence and the fluctuating wall pressure. Primary attention was given to measuring the in-stream fluctuating pressure and velocity and the correlation of each with the wall pressure.

The concentric annular turbulent flow field was shown to be consistent with information presented in the literature. Measurements of the mean velocity profile, turbulent intensity levels of the axial and radial velocity components, spectral content of the fluctuating velocity components and shear stress were made. These data were in agreement with previous measurements in annular flow geometries. The point of zero shear stress was found to lie closer to the inner wall than the point of maximum velocity. The spectral measurements of the axial and radial velocity components were shown to be similar to those measured in pipe flow:

An analysis method is presented to obtain the hydrostatic pressure fluctuation statistics in the presence of acoustic pressure contamination. The method presented is applicable to inhomogeneous. pressure fields such as those on the two walls of an annulus. The analysis method is shown to adequately obtain the hydrostatic wall pressure statistics in the presence of an acoustic pressure contamination approximately equal to the hydrostatic pressure fluctuations. The hydrostatic wall pressure intensity level, normalized by the . dynamic head $\left(\mathrm{P}_{\mathrm{rms}} / 0.5 \circ \mathrm{U}_{\mathrm{max}}^{2}\right)$, is approximately 0.0048 on the inner wall and 0.0053 on the outer wall. The spectral analysis of the wall pressure data shows that the increased intensity level is due to more low frequency pressure fluctuations on the outer wall. The PSD measurements show the energy content. per unit frequency at low frequencies is constant with the magnitude being a function of the geometry. The high frequency fluctuations are independent of the geometry and are shown to fall off with frequency at a $-9 / 3$ slope.

The in-stream bleed pressure transducer was shown to function well in the confined flow field. The intensity level of the fluctuating static pressure was found to bc eimilar in profile to the fluctuating velocity field. The numerical 
information generated by Schumann ${ }^{71}$ was in excellent agreement with the measured results of the pressure field. However, Schumann's velocity information was found to be considerably larger in magnitude than that found experimentally. The in-stream pressure transducer information near the walls was shown to be in good agreement with the wall pressure transducer information in both magnitude and spectral. content.

Pressure-pressure correlations were measured with the in-stream pressure transducer and the wall pressure transducers. The correlations were found to be strong:but significantly different on the two dissimilar wall surfaces. The axial separation correlations showed that the pressure fluctuations were being convected at approximately the mean fluid velnoity.

A strong correlation between the velocity field and the wall pressure was found. The correlation of the axial velocity component with the wall pressure was larger than the correlation of the radial velocity component with the wall pressure. Both velocity component correlations with the wall pressures were found to drop off faster at the inner wall than at the outer wall, which was similar to the results of the pressure-pressure correlations. The angular separation correlations suggested that the flow field contains a certain amount of vorticity which was approximately the same physical size near both walls.

Linear estimation theory was applied to the pressure-velocity measurements to. estimate the velocity field which contributes to the wall pressure fluctuations. The velocity field was found to be strongly related to the shear gtrees which suggest's that the largest contribution to the wall pressure may be due to. the mean shear stress interartinn with tho turbulcnee. This velucley fleld, along. with measurements of the delay time to maximum correlation, was found to be consistent with a pressure-velocity interaction in which the axial veluctty . component drives the pressure fluctuations through a Bernoulli effect and then this pressure fluctuation drives the radial velocity component. The resultant: radial velocity component would then interact with the mean flow leading to some sort of feedback mechanism. Although this type of mechanism is not believed to be the sole interaction between pressure and velocity it does appear to be the dominating mechanism. 
The correlations, with axial separation showed that the pressure-velocity interaction was convected at the mean fluid velocity which was the same as the results of the pressure-pressure correlations.

The pressure statistics obtained in this investigation can be directly employed in flow-induced vibration models which use the fluctuating hydrostatic pressure field as the driving mechanism. The information at low frequencies is particularly useful in as much as this information is new and it is in the frequency range of the vibrations. The difference in the PSD and RMS level of the two dissimilar walls shows the necessity of obtaining information in the flow geometry under analysis as the statistics from two different geometries are not interchangeable.

The wall pressure statistics have been shown to be closely related to the flow field statistics. The strongest relationship occurs between the wall pressure and the fluid shear stress. The pressure-velocity correlations and the pressure-pressure correlations yield integral length scales that are significantly larger than the boundary layer thickness. The resultant implication is that the turbulent core region makes a significant contribution to the pressure field on the wall of the flow channel. The dissimilar core flow statistics near the two walls of the annulus lead to different wall pressure sources which result in dissimilar wall pressure PSD's. The result shows that vibration modeling must incorporate the effects. of the flow geometry on the wall pressure statistics which are used as the driving force for flow-induced vibrations. In the vibration modeling of complex geometries the substitution of data from simplified flow channels may lead to erroneous results.

Schumann's ${ }^{71}$ numerically generated pressure statistics are in good agreement with the experimental results obtained in this investigation. The pressure intensity levels at the walls and the intensity profile across the flow channel are in exrel.lent agreement. The pressure-pressure correlations which could be compared with the numerical results were also in excellent agreement. The experimentally measured turbulent velocity intensity was found to be considerably less than that predicted by Schumann's ${ }^{71}$ numerical code. 
The application to flow-induced vibrations could be enhanced by considering more complex geometries or by allowing vibrations of the inner rod of the annular flow system to occur (i.e., a low stiffness rod). A current investigation (private communication with W. Nagel) of the flow field pressure and velocity effects on wall pressure in an eccentric annulus is being performed at the University of Illinois at Urbana-Champaign. This investigation should yield information which is more closely related to the results one would expect in tube bundles of heat exchangers and reactor fuel elements assemblies.

Future work which would be valuable in better understanding the turbulence in . anllular flows, should include the additional measurement of the azimuthal. velocity component and its correlation with the wall pressure. The correlafion as a function of time between the azimuthal velocity component and the wall pressure would make it possible: to generate the velocity field in the $z-i \phi$ plane using the linear estimation theory out 1,ined in Subsection 5.2. This velocity field in conjunction with that presented in the $r-z$ plane would give a pseudo three-dimensional image of the velocity field. The measurement of the velocity-velocity correlations would also be valuable in comparison with the pressure-velocity and pressure-pressure correlations measured in this investigation. These measurements would complete the experimental information obtainable in this specific flow field.

In terms of studying the pure fluid mechanics, it appears that the use of the pressure-velocity correlations, either by long time averages, conditional averages or in narrow frequency bands, could be a powerful tool. This approach has been virtually untried and there are an uncountable number of flows in which this turbulence analysis method conld he applied. 


\section{REFERENCES}

1. Wambsganss, M.W., 1967, "Vibration of Reactor Core Components;"'Reactor and Fuel Processing Techology 10, 208-219.

2. Christie, J.J. (editor), 1974, "Factors Responsible for Delay in Getting Nuclear Power in Line," Nuclear Industry, 21(4):12-13.

3. Mulcahy, T.M., Wambsganss, M.W., 1976, "Flow Induced Vibration of Nuclear Reactory System Components," Shock and Vib. Digest, 8:33-345.

4. Abramson, H.N., 1967, "Hydroelasticity - Some Problems and Some Solutions," Fluid Solid Interaction ASME, P. 187.

5. Chen, S.S., 1974, "Parallel Flow-Induced Vibrations and Instabilities of Cylindrical Structures," Shock and Vib. Digest, 6:2-12.

6. Hartog, J.P.D., 1956, "Self-Excited Vibrations," Mechanical Vibrations, 4th ed., McGraw-Hill, Chap. 7, pp. 282-329.

7. Heller, S.R., 1964, "Hydroelasticity," Advances in Hydroseiences, Academic Press, p. 94.

8. Mulcahy; T.M., Chen, S.S., 1974, "Annotated Bibliography on Flow Induced Vibrations," ANL-CT-74-05.

9. Naudascher, E., 1967, "From Flow Instability to Flow-Induced Excitation," J. of Hydraulies Division, Proc. ASCE 91 (MY4), 15.

10. Toebes, G.H., 1965, "Flow-Induced Structural Vibrations," J. Eng. Mech., Proc. ASCE 91 (EM6), 39.

11. Ananthanarayanan, K.S., 1978, Preliminary Design Handbook for Flow. Induced Vibration of Light Water Reactors," (GEAP-24158).

12. Burgreen, D., Byrnes, J.J., Benforado, D.M., 1958, "Vibration of Rods Induced by Water in Parallel Flow," Trans, ASME, 80(5):991-1003.

13. Cali, G.P., Grillo, Y., Testa, G., 1970, "Fuel Rod and Bundle Vibrations Induced by Coolant," Trans. ANS, 13(1) $333-334$.

14. Chen, Y.N., 1970, "Turbulence-Induced Instability of Fuel Rods in Parallel Flow," Sulzer Technical Review (Switzerland), 72-84.

15. Chen, Y.N., 1970, "Flow-Induced'Vibrations in Tube Bundle Heat Exchangers With Cross and Parallel Flow, Part I," Parallel Flow, Proc. Flow-Induced Vibration in Heat Exchangers (ASME), 57-66.

16. Chen, S.S., 1975, "Vibration of Nuclear Füel Bundles," Nuc, Eng. Design, $35: 399-422$.

17. Kadlec, J., Ohlmer, E., 1971, "On" the Reproducibility of the ParallelFlow-Induced Vibration of Fuel Pins," Nuc. Eng. \& Design, 17:355-360. 
18. Kadlec, J., Appe1t, K.D., 1970, "Flow-Induced Rod Vibrations of Fast Reactor Subassemblies," Nuc. Eng. \& Design, 14:136-150.

19. Paidoussis, M.P., 1965, "The Amplitude of Fluid-Induced Vibration of Cylinders in Axial Flow," AECL-2225.

20. Paidoussis, M.P., 1969, "An Experimental Study of Vibration of Flexible Cylinders Induced by Nominally Axial Flow," Nuc. Sci. \& Eng., 35:127-138.

21. Pavlica, R.T., Marshall, R.C., 1966, "An Experimental Study of Fue1 Assembly Vibrations Induced by Coolant Flow," Nuc. Eng. \& Design, $4: 54-60$.

22. Quinn, E.P., "Vibration of Fuel Rods in Parralle1 Flow," 1962, (GEAP-4059).

23. Quinn, E.P., "Vibration of SEFOR Fuel Rọds in Parallel Flow," 1965, (GEAP-4966).

24. 'Takada, Y., Egusa, T., 1968, "Vibration of the Fuel Assembly of a Marine Reactor," Nuc. Eng. \& Design, 7:578-584.

25. Randa11, R.L., 1970, "Application of Noise Analysis Techniques to Study of Flow Induced Vibration in Reactor System Components," ANL-7685, pp. 177-204.

26. Addae, A.K., Fenech, H., 1968, "Experimental Determination and Analysis of Vibrations Induced by Flow Noise in Tubes," Trans. ANS, 10:651.

27. Addac, L.K., Fenech, H., 1970, "Experimental Determination and Analysis of Vibrations Induced by the Near-Field Flow Noise in Tubes," ANL-7685, pp. 32-46.

28. Basile, D., Faure, J., Ohlmer, E., 1968, "Experimental Study on the Vibration of Various Fuel Rod Models in Parallel Flow," Nuc. Eng. \& Design, 7:517-534.

29: . Chen, S.S., Wambsganss, M.W., 1972, "Paralle1-F1nw-Tnduged Vibration of Fuel Kods," Nuc. Eng. \& Design, 18:253-278.

30. Chen, S.S., Wamhsganss, M.W., 1970, "Response of a Flex1ble kod to Nearfield Flow Noise," ANL-7685, pp. 5-31.

31. Gorman, D.J., 1969, "The Role of Turbulence in the Vibration of Reactor Fuel Elements in Liquid Flow," AECL-3371.

32. Kanazawa, R.M., 1969, "Hydroelastic Vibration of Rods in Parallel Flow," Ph.D. Thesis, University of Illinois; Urbana.

33. Knudson, S.A., Smith, G.M., 1970, "Dynamic Response of Siender Tubes in Parallel Flow Induced Vibration in Reactor System Components," ANL-7685; pP. 64-90. 
34. Mulcahy, T.M., Yeh, T.T., Miskevics, A.J., 1979, "Turbulence and Rod Vibrations in an Annular Region with Upstream Disturbances," GEAP-24173 (ANL-CT-79-12).

35. Ohlmer, E., 1973, "Experimental Investigation of an Analytical Model for Parallel Flow Induced Vibrations of 'Rod Structures," Proc. Inti. Symp. Vib, Problems in Indus., Paper No: 522, Keswick, England.

36. Ohlmer, E., Russo, S., Schwemmle, R., 1972, "Investigation of an Analytical Model for Parallel Flow Induced Rod Vibrations," Nuc. Eng. \& Design, 22: 272-289.

37. Ortloff, C.R., Ives, J., 1969, "On the Dynamic Motion of a Thin Flexible Cylinder in a Viscous Stream," J. Fluid Mech., 38:713-720.

38. Paidoussis, M.P., 1968, "Stability of Towed, Totally Submerged Flexible Cylinders,"J. Fluid Mech., 34:273-287.

39. Reavis, J.R., 1969, "Vibration Correlation for Maximum Fue1-Element Displacement in Parallel Turbulent Flow," Nuc. Science \& Engineering, $38: 63-69$.

40. Wambsganss, M.W. Jr., Boers, B.L., 1968, "Parallel-Flow-Induced Vibration of a Cylindrical Rod," Paper No. 68 - WA/NE-15.

41. Bakewell, H.P., Lumley, J.L., 1967, "Viscous Sublayer and Adjacent Wall Region in Turbulent Pipe Flow," Phys, Fluids, 10:1880.

42. Bakewe11, H.P., 1968, "Turbulent Wall-Pressure Fluctuations on a Body of Revolution," J. Acousticai Society of America, 43:1358-1363.

43. Batchelor, G.K., 1951, "Pressure Fluctuations in Isotropic Turbulence," Proc. Cambridge Phil. Soc., 47:359.

44. Blackwelder, R.F., Kaplan, R.E., 1976, "On the Bursting Phenomenon Near the Wall in Bounded Turbulent Shear Flows," J. Fluid Mech:, 76:89.

45. Blackwelder, R.F., Kovasznay, L.S.G., 1972, "Time Scales and Correlations in a Turbulent Boundary Layer," Phys. Fluids, 15:1545.

46. Blake, W.K., 1970, "Turbulent Boundary Layer Wa11 Pressure Fluctuations on Smooth and Rough Walls," J. Fluid Mech., 44:637.

47. Bu11, M.K., 1967, "Wa11 Pressure Fluctuations Associated with Subsonic Turbulent Boundary Layer Flow," J. Fluid Mech.; 28:719.

48. Bul1, M.K., Thomas, A.3.W., 1976, "High Frequenry.Wal1-Pressure Fluctuations in Turbulent Boundary Layers," Phys. Fluids, 19:597.

49. Burton, T.E., 1973, "Wal1 Pressure Fluctuations at Smooth and Rough Surfaces Under Turbulent Boundary Layers with Favorable and Adverse Pressure Gradients," Acoustics and Vibration Lab, MIT, Rep. No. 70208-9. 
50. Corcos, G.M., 1963, "Resolution of Pressure in Turbulence," J. Acoust. Soc. Am., 35:192.

51. Corcos, G.M., 1964, "The Structure of the Turbulent Pressure Field" in Boundary-Layer Flows," J. Fluid Mech., 18:353.

52.. Corino, E.R., Brodkey, R.S., 1969, "A Visual Investigation of the Wall Region in Turbulent Flow," J. Fluid Mech., 37:1.

53. Eckelmann, H., 1974, "The Structure of the Viscous Sublayer and the Adjacent Wall Region in a Turbulent: Channel Flow," J. Fluid Mech.,. $65: 439$.

54. Elliot, J.A., 1972, "Microscale. Pressure Fluctuations Measured Within the Lower Atmospheric Boundary. Layer," J. Fluid Mech., 53:351.

55. Emmerling, P., Meier, G.E.A., and.Dinkelacker, A., 1973, AGAKD Conf. Nulse Mech. Prep. No. i31.

56. Faviee, A., Gavlg1lu, J., and Dumaś, K., $193 /$, "Space-Time Double Correlations and Spectra in a Turbulent Boundary Layer," J. Fluid Mech., $2: 313$.

57. Favre, A., Gaviglio, J., and Dumas, R., 1958, "Further. Space-Time Correlations of Velocity in a Turbulent Boundary Layer," J. Fluid Mech.; $3: 344$.

58. Grotzbach, G., 1977, "Convective Velocities of" Wall Pressure Fluctuations in a Turbulent Channel Flow Deduced From a Computer-Generated Movie," Symposium on Turbulence, Berlin, Germany, Aug. 1-5, 1977.

59. Grotzback, G., Schumann, U., 1977, "Direct Numerical Simulation of Turbulent Velocity-, Pressure-, and Temperature-Fields in Channe1 Flows," Symposium on Turbulent Shear Flows, Penn State Univ., April $18-20,1977$.

60. Kraichnan, R.H., 1956, "Pressure Field Within Homogenéous Anisotropic Turbulence," eT. Acoust, Soc. Am., 28:1,64.

bl. Kraichnan, R.H., 1956, "Pressure Fluctuations in Turbulent Flow Over a Flat Plate," J. Acoust. Soc. Am., 28:378.

62. Kovasznay, L.S.G., 1970, "The turbulent Boundary T.ayer," Annu. Ren. Fluid Mech., 2:95-112:

63. Kovasznay, L.S.G., Kibens, V. and Blackwelder, R.F., 1970, "Large Scale Motion in the Intermittent Region of a Turbulent Boundary Layer, J. Fluid Mech., 41:283.

64. L̇andah1, M., 1967, "A Wạve Guide Model for Turbulent Shear Flów," J. Fluid Mech., 29:441.

65. Laufer, J., 1951, "Investigation of Turbulent Flow in a Two-Dimensiona1 Channel," NACA Rep. 1053. 
66. Lilley, G.M.,.Hodgson, T.H., 1960, "On Surface Pressure Fluctuations: in Turbulent Boundary Layers," AGARD Rep. 276.

67. Lu, S.S., and Willmarth, W.W., 1973, "Measurements of the Structure of . the Reynolds Stress in a Turbulent Boundary Layers," J. Fluid Mech., $60: 481$.

68. Panton, R.L., Linebarger, J.H., 1974, "Wa11 Pressure Spectra Calculations for Equilibrium Boundary Layers," J. Fluid Mech., 65:261.

69. Perry, A.E., Abell, C.J., 1975, "Scaling Laws for Pipe-Flow Turbulence," J. Fluid Mech., 67:257.

70. Perry, A.E., Abell, C.J., 1977, "Asymptotic Similarity of Turbulence Structures in Smonth- and Rough-Walled Pipes," J. Fluid Mech., 79:785.

71. Schumann, U., 1975, "Numerical Investigation of the Wa11 Pressure Fluctuations in Channel Flows," Nuc. Eng. Des., 32:37-46.

72. Serafini, J.S., 1963, "Wa11 Pressure Fluctuation and Pressure Velocity Correlations in Turbulent Boundary Layers," AGARD Rep. 453.

73. Wambsganss, M.W., Zaleski, P.L., 1970, "Measurement Interpretation and Characterization of Nearfield Flow Noise," ANL-7685, pp. 112-140.

74. Willmarth, W.W., 1956, "Wall Pressure Fluctuations in a Turbulent Boundary Layer," J. Acoust. Soc. Am., 28:1048.

75. Willmarth, W.W., 1958, "Space-Time Correlations of the Fluctuating Wall Pressure in a Turbulent Boundary Layer," J. Aerosp. Sci., 25:335.

76. Willmarth, W.W., Wooldridge, C.E., 1962, "Measurements of the Fluctuating Pressure at the Wall Beneath a Thick Turbulent Boundary Layer," J. Fluid Mech., 14:187.

77. Willmarth, W.W., Wooldridge, C.E., 1963, "Measurement of the Correlation Between the Fluctuating Velocities and Fluctuating. Wall Pressure in a Thick Turbulent Boundary Layer," AGARD Rep. 456.

78. Willmarth, W.W., Roos, F.W., 1965, "Resolution and Structure of the Wall Pressure Field Beneath a Turbulent Boundary Layer," J. Fluid Mech., $22: 81$.

79. Willmarth, W.W., Tu, B.J., 1967, "Structure of Turbulence in the Boundary Layer Near the Wa11," Physics of Fluids Supplement, p. 3134.

80. Willmarth, W.W., Yang, C.S., 1970, "Wa1l-Pressure Fluctuations Beneath Turbulent Boundary Layers on a Flat Plate and a Cylinder," J. Fluid Mech., 41:47.

81. Willmarth, W.W., 1971, "Unsteady Force and Pressure Measurements," Ann. Rev. Fluid Mech., 3:147. 
82. Willmarth, W.W., 1975, "Pressure Fluctuations Beneath Turbulent Boundary Layers," Ann. Rev. Fluid Mech., 7:13.

83. Willmarth, W.W., 1975, "Structure of Turbulence in Boundary Layers," Advances in Applied Mechanics, Vo1. 15, (edited by S.C. Yih), Academic Press, Inc., NY.

84. Willmarth, W.W., Winkel, R.W., Sharma, L.K., Bogar, T.J., 1976, "Axial Symmetric Turbulent Boundary Layers on Cylinders: Mean Velocity Profiles and Wall Pressure Fluctuations," J. Fluid Mech., 76:35-64.

85. Willmarth, W.W., Bogar, T.J., 1977, "Survey and New Measurements of Turbulent Structure Near the Wall," Physics of Fluids, Vo1. 20, No. 10, 59-521.

86. Willmarth, W.W., 1979, "Survey of Multiplo Sonoor Measurements atud Correlations in Boundary Layers," Coherent Structures of Turbulent Boundary Layers, Edited by Smith, C.P. and Abbott, D.E., pp. 130-167 Cunf. T.ehigh Univereity, Bcthleham, TA.

87. W11ls, J.A.B., 1964, "On Convection Velocities in Turbulent Shear Flows," J. Fluid Mech., 20:417.

88. Wyngaard, J.C., 1969, "Spatial Resolution of the Vorticity Meter and Other Hot-Wire Array," J. Scientific Instruments (J. of Physics E) Series 2, $2: 983$.

89. Baumann, H.D., 1970, "On the Prediction of Aerodynamically Created Sound Pressure Leve1 of Control Valves," ASME Pap. 7nWA/FE-28:

90. Brueggeman, W.H., Faulkner, L.L., 1975, "Acoustic Transmission of Pipe Wrapping Syotcms," AGME Pap. 7 JWA/PW-7.

91. Curle, N., 1955, "The Influence of Solid Boundaries Upon Aerodynamic Sound," Proc. R. Soc, London Ser. A 231:505-514.

92. Ffowcs-Williams, J.E., 1969, "Hydrodynamic Noise," Ann. Rev. Fluid Mech., 1:197-222.

93. Hale, M.E., Kugler, B.A., 1975, "The Acoustic Performance of Pipe Lagging Sy3tems," A3ME Rep. 75WA/Pet-2.

94. Heymann, F.J., 1973, "Some Experiments Concerning Control Valve Noise," Proc. Nnise Couf. 73, pp. 382-388.

95. Hynes, K.M., 1971, "The Development of a Low-Noise Constant Area Throttling Device," ISA Trans., 10:416-421.

96. Ingard, K.U., Slnghal, V.K., 19/4, "Sound Attenuation in Turbulent Pipe Flow," J. Acoust. Soc. Am., 55:535-538.

97. Izmit, A., 1976, "Hot-Film Anemometer-Wall Pressure FluctuationsCorrelations Related to Valve Noise," M.S. Thesis, Penn State University, 256 pp. 
98. Izmit, A., McDaniel, O.H., Reethof, G., 1977, "The Nature of Noise Sources in Control Valves," Proc. Inter-Noise 77, Zurich, Switzerland, pp. B183-188.

99. Karvelis, A.V., 1975, "An Experimental Investigation of the Wal1 Pressure Fluctuations in Piping Containing Simple Control Devices," $\mathrm{Ph} . \mathrm{D}$. Thesis, Penn State University, $256 \mathrm{pp}$.

100. Lighthill, M.J., 1952, "On Sound Generated Aerodynamically. I - Genera1 Theory," Proc. R. Soc. London Ser. A 211:564-587.

101. Lighthil1, M.J., 1954, "On Sound Generated Aerodynamically, II Turbulence as a Source of Sound," Proc. R. Soc. London Ser. A 222:1-32.

102. Morfey, C.L., 1970, "Sound Transmission and Generation in Ducts with Flow," J. Sound Vib., 14:37-55.

103. Morfey, C.L., 1973, "Amplification of Aerodynamic Noise by Convected Flow Inhomogeneities," J. Sound Vib., 31:391-397.

104. Morse, P.M., 1939, "The Transmission of Sound Inside Pipes," J. Acoust. Soc. Am., 11:205.

105. Munger, P., Gladwe11, G.M.L., 1969, "Acoustic Wave Propagation in a Sheared Fluid Contained in a Duct," J. Sound Vib., 9:28-48.

106. Munger, P., Plumblee, H.E., 1969, "Propagation and Attenuation of Sound in a Soft Walled Annular Duct Containing a Sheared Flow," Proc, NASA Basic Res. Conf., Washington, DC, pp. 305-327.

107. Pridmore-Brown, D.C., 1958, "Sound Propagation in a Fluid Flowing Through an Attenuating Duct," J. Fluid Mech., 4:393-406.

108. Reethof, G., Karvelis, A.V., 1972, "Control Valve Noise and Its Reduction, State of the Art," Proc. Inter-Noise 72, Washington, DC, pp. 146-153.

109. Robertson, J.E., 1976, "Fluid Dynamir. Noise Generation by Control Valves," Proc. Noise EXPO 76.

110. Savkar, S.D., 1971, "Propagation of Sound in Ducts with Shear Flow," J. Sound Vib., 19:355-372.

111. Sawley, R.J., 1975, "Acoustical Energy Decay in Piping Systems," ASME Pap. 75 WA/Pet-6.

112. Sawley, R.J., White, P.H., 1974, "The Influence of Pressure Recovery on the Development of Valve Noise Descriptions," ISA Pap. No. 834 presentcd at 1974 Ann. Meet. Instrum. Soc. Am.

113. Schuder, C.B., 1971, "Control Valve Noise-Prediction and Abatement," Proc. of Purdue Noise Control Conf., West Lafayette, Indiana, pp. $8 y-93$. 
114. Seebold, J.G., 1971, Valve Noise and Piping System Design Flow - Its Measurement and Control in Science and Industry, ed. R.B. Dowdell, pp. 1151-1161, Pittsburgh: Instrum. Soc. Am.

115. Sma11, D.J., Davies, P.O.A.L., 1975; "A Computerized Valve Noise Prediction System," Noise Control Eng., 4:124-128.

116. Stiles, G.T., 1974, "Reducing Fluid Transfer Noise,". Mach. Des., $46(26): 62-67$.

117. Tack, D.H., Lambert, R.F., 1965, "Influence of Shear Flow on Sound Attenuation in a Lined Duct," J. Acoust. Soc. Am., 38:655.

118. Zarate, R.A.; 1974, "Control Valve Noisé Prediction and Sizing," ISA Pap. 74-835 presented at the I\&A - 74 Conf. and Exhib., New York.

119. Reethot, G., 1978, "Turbulence-Generated Noise in Pipe Floẉ," Ann. Rev. Fluid Mech., 1.978, 10:333-367.

120. Brighton, J.A., Jones, J.B., 1964, "Fu1ly Developed Turbulent Flow in Annul1," ASME Paper No. 64-FE-2, presented at Fluids Engin. Conf., Philadelphia, PA.

121. Alster, M., 1972, "Improved Calculation of Resonant Frequencies of Helmholtz Resonators," J. Sound and Vib., 24:63-84.

122. Bainter, J.R., 1976; "Active Filter Has Stable Notch, and Response Can Be Regulated," Electronics - Designers Casebook, McGraw-Hi11, New York, pp. $175-177$.

123. Spencer, B:W., Jones, B,G., 1971, "A Blccd-Type Pressure 'lransducer for In-Stream Measurements of Statir. Preseurc Fluctuations," Kev. Sc. Inst., 42:450-454.

124. Planchon, H.P., 1974, "The Fluctuating Static Pressure Field in a Round Jet Turbulent Mixing Region," Ph.D. Thesis, Nuclear Engineering Program, Univ. of Illinois at Urband-Champaign.

125. Champagne, F.H., 1965, "Turbulence Measurements With Inclined Hot Wires," BSRT, Flight Sci. Rept. 103, DI-8i-0491.

126. Brighton, J.A., Jones, J.B., 1964, "Fully Developed Turbulent Flow in Annuli, J. Basic Eng., 86:835-844.

127. Kays, W.M., Leung, E.Y., 1963, "Heat Transfer in Annular Passagcs Hydrodynamically Developed Turbulent 'Flow With Arbitrarily Prescribed Heat Flux," Int. J. Heat Mass Transfer, 6:537-557.

128. Schlichting, H., 1968, "Boundary Layer Theory," McGraw-Hill, Inc.

129. Lawn, C.J., Elliott, C.J., 1972, "Fully Developed Turbulent .Flow Through Concentric Annuli," J. Mech. Eng. Science, 14:195-204. 
130. Lawn, C.J., E1liott, C.J., 1971, "Fully Developed Flow Through Concentric Annuli," C.E.G.B. Report RD/B/N1878.

131. Rehme, K., 1975, "Turbulence Measurements in Smooth Concentric Annuli With Small Radius," J. Fluid Mech., 72:189-206.

132. Lawn, C.J., 1971, "The Determination of the Rate of Dissipation in Turbulent Pipe Flow," J. Fluid Mech., 48:477.

133. Bakewe11, H.P., 1968, "Turbulent Wall Pressure Fluctuations on a Body of Revolution," J. Acoustic. Soc. Am., 43:1358-1363.

134. Clinch, J.M., 1969, "Measurements of the Wall Pressure Field at the Surface of a Smooth-Walled Pipe Containing Turbulent Water Flow," J. Sound Vib., 9:398-419.

135. Shin, Y.W., 1978, "Two Phase Flow-Induced Vibrations of Rods in Parallel Flow: A State of the Art Review," GEAP-24148.

136. Grotzbach, G., 1977, "Convective Velocities of Wall Pressure Fluctuations in a Turbulent Channel Flow Deduced from a ComputerGenerated Movie," Symposium on Turbulence, Ber1in, August 1-5, 1977.

137. Hansen, A.G., 1967, Fluid Mechanics, John Wiley and Sons, Inc., New York, NY.

138. Favre, A.J., Gaviglio, J.J., Dumas, R., 1957, "Space-Time Double Correlations and Spectra in a Turbulent Boundary Layer," JFM 2:313-342.

139. Adrian, R.J., 1978, "Structural Information Obtained from Analysis Using Conditional Vector Events: A Potential Tool for the Study of Coherent Structures," Coherent Stmuctures of Turbulent Boundary Layers, Edited by Smith, C.P. and Abbott, D.E., pp. 416-422, Conf. Lehigh University, Bethlehem, PA. 


\section{DISTRIBUTION LIST}

Dr. Thomas Baisa

Engineering Mechanics Programs

Corporate Research \& Development

General Electric Company

P.0. Box 8

Schenectady, New York 12301

Mr. Jon Borg

Reactor Programs Division

9800 South Cass Avenue

Argonne, Illino is 60439

Mr. J. N. Calvin

C-E Power Systems

Combustion Engineering, Inc.

1000 Prospect Hill Road

Windsor, Connecticut 06095

\section{Chief}

Mechanical Engineering Branch

Division of System Safety

U.S. Nuclear Regulatory Commission

Washington, D.C. 20555

Mail Stop P-1114

Mr. Thomas N. Rodeheaves

Associate Director for Reactors

Division of Naval Reactors

U.S. Department of Energy

Washington, D.C. 20545

Mail Stop $\mathrm{H}-404$

Mr. R. W. Curtis, Manager

Design and Development Laboratory

Alliance Research Center

Babcock \& Wilcox Company

Alliance, On io 44601

Mr. Dominic Dilanni

Engineering Branch

Division of Operating Reactors

U.S. Nuclear Regulatory Commission

Washington, D.C. 20555

Mail Stop P-1030

Mr. William Farmer

Division of Reactor Safety Research

U.S. Nuclear Regulatory Commission

Washing ton, D.C. 20555

Mail Stop 1130SS
B. Fritz

General Electric Company

Box 1072

Schenectady, NY 12301

Dr. Donald R. Harris

Department of Nuclear Engineering

Rensselaer Polytechnic Institute

Troy, New York 12181

Professor Barclay G. Jones

Nuclear Engineering Program

University of Illinois at Urbana-Champaign

214 Nuclear Engineering Laboratory

Urbana; Illinois 61801

Dr. Peter M. Lang, Chief (2)

Light Water Reactor Branch

Div. of Nuclear Power Development

U.S. Department of Energy

Washington, D.C. 20545

Mail Stop B107

Mr. R. P. Letendre

C-E Power Systems

Combustion Engineering, Inc

1000 Prospect Hill Road

Windsor, Connecticut 06095

Dr. M. Levenson, Director

Nuclear Power Division

Electric Power Research Institute

$3412 \mathrm{Hillview}$ Avenue

P.0. Box 10412

Palo Alto, California 94304

Dr. W. Loewenstein, Director

Sa fety and Analysis Department

Electric Power Research Institute

3412 Hillview Avenue

P.0. Box 10412

Palo Alto, California 94304

Mr. Barry J. Lubin

C.-E Power Systems

Combustion Engineering, Inc.

1000 Prospect Hill Road

Windsor, Connecticut 06095

Mail Code 9485-1027 
Ms. Jane Monhart/Mr. Lou Parys (2 copies)

Contracts Management Office

U.S. Department of Energy

Chicago Operations Office

9800 South Cass Avenue

Argonne, Illinois 60439

Dr. T. M. Mulcahy

Vibration Analysis Section

Components Technology Division

Argonne National Laboratory

9700 South Cass Avenue

Argonne, Illinois 60439

Professor M. P. Paidoussis, Chairman

Department of Mechanical Engineering

McGill University

817 Sherbrook Street West

Montrea 1, P.W., Canada H3A2K6

Dr. M. K. Au -Yang

Babcock \& Wilcox

Nuclear Power Generating Division

P. O. Box 1260

Lynchburg, VA 24505

Professor Robert Plunkett

Department of Aerospace

Engineering \& Mechanics

University of Minnesota

107 Aeronautics Engineering Building

Minneapolis, Minnesota 55455

Mr. Joseph A. Prestele

Assistant Department Director

Nuclear Engineering \& .

Operations Department

Electric Power Research Institute

3412 Hillview Avenue

P.O. Box 10412

Pallo Alto, California 94304

E. Ruggiero

Code 08

Department of the Navy

Naval Sea Systems Command

Washington, D.C. 20.362

Department of Energy (183)

Technical Information Center

P.O. Box 62

Oak Ridge, TN 37830
Dr. Sudhir Savkar

Engineering Mechanics Programs

Corporate Research \& Development

General Electric Company

P.0. Box 8

Schenectady, New York 12301

Mr. Norman Singleton

Westinghouse Electric Corp. P.0. Box 355

Pittsburgh, PA 15230

Dr. Ronald So

Engineering Mechanics Programs

Corporate Research \& Development

General Electric Company

P.0. Box 8

Schenectady, New York 12301

Dr. Martin W. Wambsganss

Viluraliun Analysis Section

Components Technology Division

Argonne National Laboratory

9700 snuth Cass Avenue

Argonne, Illinois 60439

$\mathrm{Mr}$. Jack Patterson

Exxon Nuclear

2101 Horn Rapids Road

Richland, Washington 99352

Dr. Randall J. Wilson

Reactor Analysis and Safety Division

Argonne National Laboratory

9700 South Cass Avenue

Argonne, Illinois 60439

Dr. Robert J. Hansen

Naval Research Laboratory

Mail Code 8444

Washington, D.C. 20375

Professor W.W. Willmarth

University of Michigan.

Department of Aerospace Engineering

Aerospace Engineering Building

Ann Arbor, Michigan 48109. 